\title{
On the Intelligent Management of Sepsis in the Intensive Care Unit
}

\author{
Vicent J. Ribas Ripoll \\ e-mail: vribas@lsi.upc.edu \\ Supervisors: Dr. Alfredo Vellido Alcacena \\ Dr. Enrique Romero Merino \\ Soft Computing Research Group \\ LSI-Department (Artificial Intelligence Section) \\ Universitat Politècnica de Catalunya
}

October 25, 2012 
O Rose, thou art sick!

The invisible worm

That flies in the night,

In the howling storm,

Has found out thy bed

Of crimson joy:

And his dark secret love

Does thy life destroy.

William Blake 


\section{Contents}

1 Introduction $\mathbf{1 5}$

1.1 Motivation .................... . . 16

1.2 Thesis Objectives . . . . . . . . . . . . . . . . . 16

1.3 Considerations about the Analysed Datasets . . . . . . . . . . . 17

1.4 Expected Contributions . . . . . . . . . . . . . 18

1.5 Thesis Structure . . . . . . . . . . . . . . . 18

2 Medical Background: The Sepsis Pathology 21

2.1 Phylogenetic Overview . . . . . . . . . . . . . . . . . 22

2.2 Historic Overview . . . . . . . . . . . . . . . . . . . . . . . . . . . . . . . . . . . 23

2.3 Clinical Overview . . . . . . . . . . . . . . . . 26

2.3.1 Definitions . . . . . . . . . . . . . 26

2.4 Systems for Scoring the Severity of Sepsis . . . . . . . . . . . . . 29

2.4.1 Sequential Organ Failure Assessment Score . . . . . . . . 30

2.4.2 Acute Physiology and Chronic Health Evaluation II . . . 30

3 State of the Art: Quantitative Analysis of Sepsis 35

3.1 Quantitative Analysis of the Pathophysiology of Sepsis . . . . . . 35

3.2 Quantitative Analysis of the Prognosis of Sepsis . . . . . . . . . . 37

3.3 Limitations of Existing Quantitative Analysis . . . . . . . . . . 39

4 Background: Algebraic Statistical Models, Algebraic Exponential Families and Generative Kernels $\quad 41$

4.1 Polynomial Representation: Outline in Three Examples . . . . . 41

4.1.1 Linear and Polynomial Regression . . . . . . . . . . . . 42

4.1 .2 Interpolation . . . . . . . . . . . . . . . . 42

4.1.3 Polynomial Representation of a Univariate Gaussian Vari-

able ................... 43

4.2 Algebraic Models . . . . . . . . . . . . . . . . . . . . . . . . . . . . . 44

4.2 .1 Division .................... . . 46

4.2 .2 Gröbner Bases . . . . . . . . . . . . . . . . . 47

4.2.3 Algorithm for Polynomial Regression/Interpolation of Observation Matrices . . . . . . . . . . . . . . . 48

4.3 Regular Exponential Families . . . . . . . . . . . . . . . 50

4.3.1 Important Properties of Regular Exponential Families . . 50

4.3.2 Discrete Distributions as Regular Exponential Families . . 51

4.3.3 Gaussian Distributions as Regular Exponential Families . 52

4.4 Algebraic Exponential Families . . . . . . . . . . . . . . 52 
4.4 .1 Semi-Algebraic sets . . . . . . . . . . . . . . . . . 53

4.4.2 Independence Models and Algebraic Exponential Families 54

4.4.3 Factorization of Discrete Distributions and Graphical Mod-

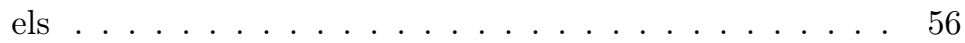

4.4.4 Markov Random Fields and Graphical Models . . . . . . 56

4.5 Kernels: Definitions and Properties . . . . . . . . . . . . . . . 59

4.5.1 Important Properties of Positive and Negative Definite Kernels . . . . . . . . . . . . . . . . . 6 60

4.5.2 Relation between Positive and Negative Definite Kernels . 61

4.5.3 Reproducing Kernel Hilbert Spaces . . . . . . . . . . . . . 62

4.5.4 Kernels as Covariance Functions . . . . . . . . . . . . . 62

4.6 Generative Kernels from Algebraic Statistical Models . . . . . . . 64

4.6.1 Quotient Basis Kernel . . . . . . . . . . . . . . . . . . 64

4.6.2 Fisher Kernel for Exponential Families . . . . . . . . . . . 65

4.6.3 Kernels based on the Jensen-Shannon metric . . . . . . . 66

5 Background: Methods for Regression, Classification and Dimensionality Reduction

5.1 Regression Trees . . . . . . . . . . . . . . . . . . . . . 69

5.2 Classification Techniques . . . . . . . . . . . . . . . . 71

5.2.1 Logistic Regression: Classification as Binomial Regression 71

5.2 .2 Support Vector Machines . . . . . . . . . . . . . . . 71

5.2.3 Classification with Feature Selection: Relevance Vector

Machines . . . . . . . . . . . . . . . . . 75

5.3 Dimensionality Reduction . . . . . . . . . . . . . . . . 77

5.3.1 Feature Selection Methods . . . . . . . . . . . . . . 77

5.4 Feature Extraction Methods . . . . . . . . . . . . . . . . 77

6 Graphical Models of Sepsis Incidence and Outcome Prediction in Patients Treated with Statins $\quad 81$

6.1 Introduction . . . . . . . . . . . . . . . . . . . 81

6.2 Materials . . . . . . . . . . . . . . . . . . . 82

6.3 Methods . . . . . . . . . . . . . . . . . . . . . . 82

6.3.1 Algebraic Statistical Models . . . . . . . . . . . . . . 82

6.3.2 Models of Conditional Independence . . . . . . . . . . . . 83

6.3.3 Markov Random Fields . . . . . . . . . . . . . . . . . 83

6.3.4 Algebraic Interpolation from Gröbner Bases . . . . . . . . 84

6.4 Results . . . . . . . . . . . . . . . . . . . . 85

6.4.1 Study of the Incidence of Sepsis with Bayes Networks over the basal SOFA Score . . . . . . . . . . . . . . . 85

6.4.2 Marginal Dependence Between Preadmission Use of Statins and the ICU Outcome . . . . . . . . . . . . . . 85

6.4.3 Study of the Protective Effect of Preadmission Use of Statins with MRFs . . . . . . . . . . . . . 90 90

6.4.4 Study of Interactions by means of Algebraic Interpolation 91

6.4.5 Study of the Protective Effect of Preadmission Use of Statins with Regression Trees . . . . . . . . . . . . . . 92

6.4.6 Study of Septic Shock Incidence with Regression Trees . . 93

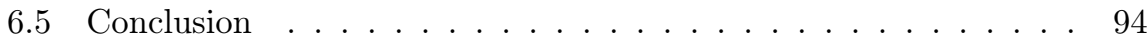


7 Severe Sepsis Mortality Prediction Using an Interpretable Latent Data Representation $\quad 97$

7.1 Introduction . . . . . . . . . . . . . . . . . . . . . . . 97

7.2 Materials . . . . . . . . . . . . . . . . . . . . 97

7.3 Results . . . . . . . . . . . . . . . . . . . . . 9 98

7.3.1 Diagnosis of the Factor Analysis Model . . . . . . . . 98

7.3.2 Factor Interpretation from a Clinical Viewpoint . . . . . . 100

7.3.3 Mortality prediction using logistic regression over 14 factors 101

7.3.4 Comparison with Logistic Regression over a Selection of the Original Variables . . . . . . . . . . . . . . . 103

7.3.5 Comparison with the APACHE II Mortality Score . . . . 104

7.4 Conclusions . . . . . . . . . . . . . . . . . . . . 104

8 Severe Sepsis Mortality Prediction from Observed Data 107

8.1 Introduction . . . . . . . . . . . . . . . . . . . . . . . 107

8.2 Materials: Detailed Description of Generative Kernels . . . . . . 108

8.2.1 Quotient Basis Kernel . . . . . . . . . . . . . . . . . . 108

8.2.2 Fisher Kernel for Exponential Families . . . . . . . . . . . 110

8.2.3 Kernels based on the Jensen-Shannon metric . . . . . . . 111

8.3 Results . . . . . . . . . . . . . . . . . . . . . . . . . . 112

8.3.1 Mortality Prediction with RVM . . . . . . . . . . . . 112

8.3.2 Comparison with Shrinkage Feature Selection Methods for

Logistic Regression . . . . . . . . . . . . . . . . . . 113

8.3.3 Mortality Prediction with Generative Kernels . . . . . . . 114

8.4 Conclusions . . . . . . . . . . . . . . . . . 116

9 Conclusions 119

9.1 On the Incidence of Sepsis and Coadjutant Factors to be Taken into Consideration . . . . . . . . . . . . . . . 120

9.2 Summary of Prognosis Indicators Obtainend and Their Accuracy 121

9.3 Summary of Mortality Predictors and Their Accuracy . . . . . . 121

9.4 Contributions . . . . . . . . . . . . . . . . . . . . 123

9.4.1 Methodological Contributions . . . . . . . . . . . . . 123

9.4 .2 Clinical Contributions . . . . . . . . . . . . . . . . 123

9.5 Publications . . . . . . . . . . . . . . . . . . . . 124

9.5.1 Publications Directly Linked to this PhD Thesis . . . . . 124

9.5.2 Relevant Information Related to this PhD Thesis . . . . . 124

9.6 Outline for Future Work . . . . . . . . . . . . . . . . 125

A General Considerations of Topology and Measure Theory 127

A.1 Topological Spaces . . . . . . . . . . . . . . . . . . 127

A.2 Measures . . . . . . . . . . . . . . . . . . . . . 129

A.3 Entropy and Divergences . . . . . . . . . . . . . . 130 


\section{List of Figures}

2.1 phylogenetic tree for the IRAK-3 Inflammation Toll Receptor . . 24

2.2 Sepsis Overview: The main sources of Sepsis is either an Infection or SIRS, after that it may evolve to Severe Sepsis, which in turn can evolve toward MODS or Septic Shock. . . . . . . . . . . . 27

2.3 APACHE II Table . . . . . . . . . . . . . . . . . . . 33

5.1 Hyperplane through two linearly separable classes. . . . . . . . . 72

5.2 Graphical Representation of the Factor Analysis Model $\mathbf{F}_{\mathbf{1 2}, \mathbf{1 0}} \quad$ • 79

6.1 APACHE II threshold selection: The blue curve represents the true APACHE II mortality rate, whilst the smooth red curve is the APACHE II mortality rate interpolated with a cubic polynomial. The arrow points to the first inflection point of the polynomial, which, in this study, corresponds to the selected APACHE II threshold for stratification (i.e. APACHE II = 21). This means that APACHE II scores lower than this threshold are set to 2 in our MRF. Conversely, the APACHE II values higher than 21 are set to 1 in our MRF. This threshold is consistent with standard clinical practice [1] . . . . . . . . . . . . . . . . . 86

6.2 SOFA Score threshold selection: The blue curve represents the true SOFA SCORE mortality rate, whilst the smooth red curve is the SOFA Score mortality rate interpolated with a cubic polynomial. As in the previous figure, the arrow points to the first inflection point of the polynomial, which is selected as SOFA Score threshold for stratification (i.e. SOFA $=7$ ). This means that SOFA scores lower than this threshold are set to 2 in our MRF. Conversely, the SOFA values higher than 7 are set to 1 in our MRF. This threshold is consistent with standard clinical practice. . . . . . . . . . . . . . . . . . 87

6.3 Regression Tree for Probability of Survival. . . . . . . . . . . . . 94

6.4 Regression Tree for Shock Prediction . . . . . . . . . . . . . . . . 94

A.1 Two points separated by open sets in a Haussdorff Space . . . . . 129 


\section{List of Tables}

2.1 SOFA Score table adapted from [2]. Here, MAP stands for Mean Arterial Pressure, DPM for dopamine, DBT for dobutamine, AD for adrenaline, and NAD for Noradrenaline. Dosages are given in $[\mu \mathrm{g} / \mathrm{Kg} \cdot \min ] . \ldots \ldots \ldots \ldots \ldots \ldots \ldots \ldots$

4.1 Contingency Table for Gröbner Basis . . . . . . . . . . . . 48

6.1 List of SOFA scores, with their corresponding mean and standard deviation values. . . . . . . . . . . . . . . . 83

6.2 Ranks of Minors Obtained with SVD . . . . . . . . . . 88

6.3 Ranks, $H_{0}:\left\{X_{1}\right\} \Perp\left\{X_{2}\right\} \mid\left\{X_{3}\right\},\left\{X_{4}\right\} \ldots \ldots \ldots \ldots$

6.4 Ranks, $H_{0}:\left\{X_{1}\right\} \Perp\left\{X_{3}\right\} \mid\left\{X_{2}\right\},\left\{X_{4}\right\} \ldots \ldots \ldots \ldots$

6.5 Ranks, $H_{0}:\left\{X_{2}\right\} \Perp\left\{X_{3}\right\} \mid\left\{X_{1}\right\},\left\{X_{4}\right\} \ldots \ldots \ldots$

6.6 Ranks, $H_{0}:\left\{X_{2}\right\} \Perp\left\{X_{4}\right\} \mid\left\{X_{1}\right\},\left\{X_{3}\right\} \ldots \ldots \ldots \ldots$

6.7 Ranks, $H_{0}:\left\{X_{3}\right\} \Perp\left\{X_{4}\right\} \mid\left\{X_{1}\right\},\left\{X_{2}\right\} \ldots \ldots \ldots \ldots$

6.8 Marginal Probabilities for ICU results . . . . . . . . . . . 91

7.1 List of SOFA scores, with their corresponding mean and standard deviation values for the population under study (scoring organ dysfunction). . . . . . . . . . . . . . . . . 98

7.2 List of variables used in this study. . . . . . . . . . . . . . 99

7.3 Loadings Matrix: $|\Lambda(i, j)|>$ quantile 95 for Factor $f_{i}$ are presented in bold. . . . . . . . . . . . . . . . . . 102

7.4 Results for LR over Latent Factors with 10-fold cross validation . 103

7.5 Results for LR with 10 -fold cross validation . . . . . . . . . 103

8.1 Results for Shrinkage Methods . . . . . . . . . . . . . . . . . 114

8.2 Results for SVM with Generative Kernels . . . . . . . . . . . 116

8.3 p-value table for the Wilcoxon Rank Sum Test. The null hypothesis tested is that the cdf for the resulting error distributions for each kernel are different . . . . . . . . . . . . . . . . . . 116

9.1 Summary of attributes, the dataset where they are used and their calculation. . . . . . . . . . . . . . . . . 122

9.2 Summary of Prognosis Indicators and their Corresponding Accuracies . . . . . . . . . . . . . . . . . . . . 123 


\section{Abstract}

The management of the Intensive Care Unit (ICU) in a hospital has its own, very specific requirements that involve, amongst others, issues of risk-adjusted mortality and average length of stay; nurse turnover and communication with physicians; technical quality of care; the ability to meet patient's family needs; and avoid medical error due rapidly changing circumstances and work overload. In the end, good ICU management should lead to an improvement on patient outcomes.

Decision making in the ICU environment is a real-time challenge that works according to very tight guidelines, which relate to often complex and sensitive research ethics issues. Clinicians in this context must act upon as much available information as possible, and could therefore, in general, benefit from at least partially automated computer-based decision support based on qualitative and quantitative information. Those taking executive decisions at ICUs will require methods that are not only reliable, but also, and this is a key issue, readily interpretable. Otherwise, any decision tool, regardless of its sophistication and accuracy, risks being rendered useless.

This thesis addresses this through the design and development of computer based decision making tools to assist clinicians at the ICU. It focuses on one of the main problems that they must face: the management of the Sepsis pathology (i.e. the systemic inflammatory response to a confirmed infection). Sepsis is one of the main causes of death for non-coronary ICU patients. Its mortality rate can reach almost up to one out of two patients for septic shock, its most acute manifestation. It is a transversal condition affecting people of all ages. Surprisingly, its definition was only standardized two decades ago as a systemic inflammatory response syndrome with confirmed infection.

The research reported in this document deals with the problem of Sepsis data analysis in general and, more specifically, with the problem of survival prediction for patients affected with Severe Sepsis. The tools at the core of the investigated data analysis procedures stem from the fields of multivariate and algebraic statistics, algebraic geometry, machine learning and computational intelligence.

Beyond data analysis itself, the current thesis makes contributions from a clinical point of view, as it provides substantial evidence to the debate about the impact of the preadmission use of statin drugs in the ICU outcome. It also sheds light into the dependence between Septic Shock and Multi Organic Dysfunction Syndrome. Moreover, it defines a latent set of Sepsis descriptors to be used as prognostic factors for the prediction of mortality and achieves an improvement on predictive capability over indicators currently in use. 


\section{Acknowledgments}

This $\mathrm{PhD}$ is essentially multidisciplinary since we are dealing with a difficult medical issue through machine learning and algebraic modelling.

First and foremost, I would like to thank Dr. Angela Nebot for accepting me to the Soft Computing group as a PhD student. I am mostly indebted to my PhD supervisors Drs. Alfredo Vellido and Enrique Romero for their great advice, support and constructive criticism.

From the clinical side, I would like to express my gratitude to Dr. Francisco de la Torre and Dr. Jordi Rello for granting me access to the ICU at Hospital Vall d'Hebron and letting me work with their exceptional team of doctors. I would also like to thank Drs. Ruíz-Rodríguez and Caballero for sharing with me their knowledge about Sepsis and also for their inconditional support in drafting the clinical studies that had to be approved by Hospital's Etical and Scientific Committee. They have provided the patient data and also revised the clinical part of this document and showed even more patience when I presented them the most technically difficult and abstract concepts of this $\mathrm{PhD}$.

I am also indebted to Dr. Marta Casanellas because she opened to me the world of algebraic modelling. I had been very reluctant to take her course in algebraic modelling in genomics during my MSc in Mathematical Engineering. Had it not been for this course and its professor, I would have never thought about the Quotient Basis Kernel or understood the role of statins during Sepsis. I am convinced that my research will be related to Algebraic Statistics for a very long time.

I would also like to thank my parents (Vicent and Maria Neus) for supporting me during most part of my studies and for giving me the values of hard work and patience. I would also like to thank most of my teachers from school and highschool for nurturing my curiosity and impressing on me the pleasure of knowledge. As one of them used to say (Mr. Lluís Busquets): "estudiar es relacionar".

And last but not least, I would like to express my deepest gratitude to my wife, Maria José, who has been the source of inspiration for this $\mathrm{PhD}$ thesis. This is definitely the result of her ability to come up with really difficult problems and her capacity to give a turn in the point of view of addressing them. She really managed to make me believe that Math must meet Medicine. I should also thank her for the patience that she has shown during these long years. I hope she will forgive all the time and weekends that I have stolen from her as well as our three children: Vicent, Mar and Diana. If it had not been for their love and support, I would have never been able to complete this $\mathrm{PhD}$. 


\section{Chapter 1}

\section{Introduction}

¿Y si antes de empezar lo que hay que hacer, empezamos lo que tendríamos que haber hecho?

Mafalda

Sepsis is one of the main causes of death for non-coronary ICU (Intensive Care Unit) patients. It is a transversal condition affecting people of all ages and, more particularly, immunocompromised patients, critically ill patients, postsurgery patients, AIDS patients, and the elderly. In western countries, septic patients account for as much as $25 \%$ of ICU bed utilization and occurs in $1 \%$ $2 \%$ of all hospitalizations. The mortality rates range from $20 \%$ for Sepsis and $40 \%$ for Severe Sepsis, to over $60 \%$ for Septic Shock.

Septic response and the Systemic Inflammatory Response Syndrome (SIRS) can be portrayed as being one of the main contributing factors to around 200,000 deaths per year only in the United States. Moreover, this condition has presented a clear upwards trend for the last 20 years resulting in around 300,000 cases per year in the United States. The high rates of Severe Sepsis in western societies may be due to the ageing population, the increasing longevity of patients with chronic diseases and the relative high frequency with which Sepsis develops in patients with AIDS (immunocompromised patients) and those patients who have received an organ transplant or undergone complex surgery.

One of the main complications of the Septic Shock is that it may result in Cardiogenic Shock. Cardiovascular dysfunction resulting from Septic Shock and Cardiogenic Shock require immediate resuscitative efforts to prevent progressive end-organ damage and death. The diagnosis of Septic Shock is not trivial and it is usually carried out in challenging clinical emergency situations. Early recognition of signs of decreased perfusion before the onset of hypotension, appropriate therapeutic response, and removal of the center of the infection are the keys to survival of patients with Septic Shock. Given the criticality of Septic Shock, it is of capital importance to have available an early indication of this condition in order to allow doctors to act rapidly at the onset of Sepsis.

Needless to say, the ICU environment can be an unforgiving one in terms of decision making tasks. Clinicians in general might benefit from at least partially automated computer-based decision support, but those clinicians making realtime executive decisions at ICUs in particular will require methods that are not 
only reliable, but also, and this is a key issue, readily interpretable. This thesis aims to address these needs through the design and development of computerbased decision making tools to assist clinicians at the ICU. These developments will focus on the problem of Sepsis in general and, more specifically, on the problem of survival prediction for patients with Severe Sepsis. The tools of Sepsis data analysis in this work stem form the fields of multivariate statistics, algebraic statistics, algebraic geometry, machine learning and computational intelligence.

\subsection{Motivation}

From what has been stated above, one may conclude that Sepsis is the result of the uncontrolled inflammatory response to infection. At this stage it is also very important to note that, today, Sepsis is a health state that can only be assessed with certainty a posteriori (i.e. when the condition has already taken place), but at the same time requires action to be taken immediately and, whenever possible, preventively [3, 4]. Extensive research efforts have been made to study Sepsis from a proteomics point of view (a good overview on this topic can be found in [5]), but as of today the results are so far inconclusive and costeffectiveness of specific treatments such as Drotrecogin alpha (activated) (Xigris TM, Elli Lilly) is still under debate [6]. For this reason, it is extremely important to provide simple and readily interpretable tools to manage Sepsis and improve its prognosis.

This becomes even more important when taking into account that the ICU is an extremely data intensive environment. Monitoring ranges from beat-tobeat (Blood Pressure, Heart Rate or ECG), hours (gas exchange, white blood cell count, lactate), to days (Apache, SOFA, Dynamic SOFA). The aggregated data storage requirements for a patient can be of several Gigabytes, if we take into consideration all biomedical signals. It is therefore understandable that any new parameter to be measured in the ICU must provide high value in terms of prognosis and interpretation (i.e. must be associated with and complementary to the pathophysiology and management of Sepsis).

Moreover, there is a non-trivial relation between the parameters and clinical traits mentioned above and the different types and degrees of Sepsis that can be statistically estimated. It is also possible that different machine learning techniques can be employed to identify these relations and improve the management of the Septic patient. More particularly, the continuation of some preadmission treatments during the ICU stay may have a significant impact on outcome. In conclusion, there is a clear need to develop/modify the analytical tools for studying the prognosis of septic patients and also improve the sensitivity and specificity capabilities of the scores already available and currently in use in clinical practice, whilst keeping the overall complexity of such tools at a reasonable and practical level.

\subsection{Thesis Objectives}

The main objectives of this $\mathrm{PhD}$ thesis are:

1. Improving our knowledge about the incidence of Sepsis. Although the 
incidence of Sepsis is, in general, very well documented [3] (c.f. section 6.4.1) there is still some controversy about the real incidence of Sepsis in Spain. For example, this is one of the main issues of contention at the Hospital in which the data analysed in this thesis were generated, given the fact that they only see and therefore control the most severe cases of Sepsis (while the less severe are managed in the general ward).

2. Improving the understanding of Sepsis physiology and inferring functions that describe the relationship of measured variables with the state of Sepsis. According to the definitions of Sepsis given in the following chapters, there is a clear difference between Multiple Organ Failure Syndrome (MODS) and Septic Shock. However, very seldom does one see a pure Septic Shock without MODS (Multi Organ Dysfunction Syndrome). In other words, there must be a dependence between them and it is this relation that must play an important role in the prognosis and management of sepsis.

3. Studying the time evolution of Sepsis with respect to several management/measurement variables. The main results of the Surviving Sepsis Campaign (SSC) have also been controversial [7] due to the fact that some studies also show that the most important factors from the SSC are the timely administration of antibiotics and performance of haemocultures. Given the fact that the ICU that we collaborate with is quite compliant with the SSC, we plan to evaluate the impact of these guidelines in ICU outcome and detect which ones are the most predictive.

4. Developing a system that could provide prognostic indicators of mortality related to Sepsis, with high reliability, at the onset of the pathology. The most important indicators of Sepsis (SOFA and APACHE II) are calculated at admission to the ICU. However, there are other variables that may play an important role in the prognosis of Sepsis. Here we plan to detect the underlying factors that explain the ICU prognosis model and also perform attribute selection procedures, which may complement those used in clinical practice (backward and forward feature selection in linear/logistic regression).

\subsection{Considerations about the Analysed Datasets}

This $\mathrm{PhD}$ thesis analyses two main datasets. More specifically, the first two databases come from two independent prospective studies approved by the Clinical Investigation Ethical Committee of the Vall d'Hebron University Hospital in Barcelona, Spain. The data for these two studies was collected by the Group on Shock, Organic Dysfunction and Resuscitation (SODIR) of Vall d'Hebron's Intensive Care Unit (VH-ICU).

The first dataset is described in detail in chapter 6 and is devoted to studying the impact of the preadmission use of statins on the prognosis of Sepsis. This dataset is extremely valuable not only because it is far larger than any other reported in the literature (see chapter 6), but also because it is accompanied by the most important scores at admission. This dataset has enabled us to put the preadmission use of statins in the context of severity and organ dysfunction, 
which clearly have an impact on the interpretation and disparity of results found in the literature.

The second dataset is presented in chapter 7 and covers the time span between June 2007 and December 2010. This dataset includes 354 patients. At this stage it is also important to note that this dataset is affected in its number of patients by the flu pandemic that took place during autumn/winter 2010 .

\subsection{Expected Contributions}

The expected contributions of this thesis are twofold. From a clinical point of view, it is expected to clarify and shed some light onto the debate about the impact of the preadmission use of statins in the ICU outcome, and show the dependence between Septic Shock and Multi Organic Dysfunction Syndrome (MODS). Also, from a clinical point of view it is expected to obtain a latent model-based set of descriptors of sepsis, which could be used as prognostic factors for the prediction of mortality due to Sepsis. And last but not least, it is also expected to improve the overall accuracy of already existing prognostic indicators widely used by the clinical practice by means of variable selection, shrinkage methods and generative kernels.

From a machine learning point of view, it is expected to study the dependence relations between the different variables by means of Algebraic Statistical Models. These models, put in context of the Regular Exponential Families, will enable us to re-parametrize the probability distribution functions by means of polynomial ideals on an algebraic variety. Although this approach has been successfully deployed in phylogenetics (where different models are used to study the mutations between genes), in the approach followed in this thesis, transition matrices are calculated and parametrized from the available data. We also use a very powerful theorem (Hammersley Clifford) to study the marginal dependence between variables and obtain the associated graphical models. Finally, we show that the Algebraic Statistical Models for the Regular Exponential Family over a metric space (Haussdorff) induce a convex-dual space that can be used to derive Generative Kernels by means of a re-parametrization of the cumulant generation function to the negative entropy.

\subsection{Thesis Structure}

This thesis is organized as follows:

- Chapter 2 presents an overview of Sepsis from three different perspectives. First of all, we provide a philogenetics overview, which shows that Sepsis is a cross-species syndrome and therefore as old as mankind. Secondly, we present an historic overview, starting from the first documented case of sepsis in Plutarch. In this section, we also present the most modern definitions of Sepsis as a continuum (i.e. Infection, Inflammatory Response, Sepsis, Severe Sepsis, Shock and Multi Organic Dysfunction). This chapter is closed with a description of the Sepsis scoring systems most widely used in clinical practice. 
- Chapter 3 is devoted to a State of the Art of current quantitative and qualitative methods for the assessment of the pathophysiology and prognosis of Sepsis using machine learning techniques.

- Chapter 4 is mostly technical and provides the necessary background for Algebraic Statistical Models and Generative Kernels. In this chapter, graphical models are presented as a particular case of Algebraic Models. In this chapter we also present a new kernel derived from Quotient Bases of Algebraic Models.

- Chapter 5 provides the required background for the classification, regression and feature selection methods that we used throughout the thesis for the study of Sepsis.

- Chapter 6 is devoted to the study of the incidence of sepsis and the impact of preadmission use of Statins on the ICU outcome for septic patients. This study starts with an analysis of conditional dependence between the input variables, followed by a study of outcomes by means of algebraic models, algebraic interpolation, Graphical Models and Classification and Regression Trees.

- Chapter 7 presents our approach to Severe Sepsis Mortality prediction using an interpretable latent data representation (obtained through Factor Analysis). First we provide a latent description of our input dataset by means of Factor Analysis. The extracted factors are then used to calculate a logistic regression model for mortality prediction. This logistic regression model is compared against clinically well established state of the art methods.

- Chapter 8 deals with the application of shrinkage methods (for dimensionality reduction) with Relevance Vector Machines for the assessment of Risk of Death (ROD) and also sets all the kernels defined in Chapter 4 in action. Given that the resulting (reduced) dataset is consistent with standard clinical practice, it shall be used later on to study other ROD predictors based on Kernel Methods.

- Chapter 9 presents the conclusions of this $\mathrm{PhD}$ thesis, the publications and the main contributions (methodologic and clinical) 


\title{
Chapter 2
}

\section{Medical Background: The Sepsis Pathology}

\author{
The world, unfortunately, rarely \\ matches our hopes and \\ consistently refuses to behave in a \\ reasonable manner.
}

Stephen Jay Gould

As mentioned in the introduction, Sepsis is one of the main causes of death for non-coronary ICU patients. According to [3], it is the tenth most common cause of death. Its mortality rates can reach up to $45.7 \%$ for septic shock, its most acute manifestation. For these reasons, the prediction of the mortality caused by sepsis is an open and relevant medical research challenge.

In western countries, septic patients account for as much as $25 \%$ of ICU bed utilization and occurs in 1\% - $2 \%$ of all hospitalizations. The statistics for Catalonia (the Spanish region where the analysed data was collected) do not differ from those presented above and septic patients account for $25 \%$ of bed occupation at ICUs and PICUS (Pediatric ICUs), while approximately twothirds of septic cases take place in patients hospitalized for other illnesses.

The high rates of Severe Sepsis in western societies may be due to the ageing population, the increasing longevity of patients with chronic diseases and the relative high frequency with which Sepsis develops in patients with AIDS (immunocompromised patients) and those patients who have received an organ transplant or undergone complex surgery. According to [4], the widespread use of antibiotics, glucocorticoids, invasive catheterism and other mechanical devices (such as mechanical ventilation and extra-corporeal circulation) also play a role in the onset of Sepsis, Severe Sepsis and Septic Shock.

Patients clinically suspected of infection, an abnormal temperature and tachycardia may be diagnosed with Septic Shock if they develop at least one of the following manifestations of decreased organ perfusion: altered mental status, oliguria, delayed capillary refill, bounding peripheral pulses or increased lactate level. These clinical signs take place before hypotension. Decreased blood pressure is a late sign of Septic Shock. Early recognition of signs of decreased perfusion before the onset of hypotension, appropriate therapeutic 
response, and removal of the center of the infection are key to the survival of patients with Septic Shock. Given the criticality of this pathology, the availability of an early indication of the condition is of capital importance in order to allow doctors to act rapidly at the onset of Sepsis.

Sepsis is the local or systemic response [4] to microbiotic agents (bacteria, virus or fungus) traversing the epithelial barriers and invading the tissue underlying. The main signs of SIRS (Systemic Inflammatory Response) include fever, tachycardia and peripheral vasodilation (i.e. the inflammatory triad) as well as hypothermia, leukocytosis or leukopenia and tachypnea. The symptoms outlined above are commonly seen in patients with benign viral or bacterial infections that respond to management with antipyretics or antibiotics or both. However, signs of hypoperfusion (i.e. decreased blood flood through an organ) suggest the possibility of early Septic Shock.

According to [4]

"SIRS may have an infectious or a non-infectious aetiology. If infection is suspected or proved, a patient with SIRS is said to have Sepsis."

If Sepsis was associated with the dysfunction of organs distant to the site of infection, then the patient would be diagnosed with Severe Sepsis. Like Septic Shock, Severe Sepsis is associated with both hypotension and hypoperfusion. The impossibility of correcting the hypotension by means of fluid infusion, leads to a diagnosis of Septic Shock. As Sepsis progresses to Septic Shock, the risk of dying increases substantially. Sepsis can be reversed while patients with Septic Shock often pass away despite aggressive therapy.

The complications associated with Sepsis can be summarized as follows:

- Cardiopulmonary complications: hypoxaemia, increased pulmonary water content, decreased capillary refill, hypovolemia, acute respiratory distress syndrome (ARDS) and depression of myocardial function.

- Renal complications: decreased urine output, azotemia, proteinuria and non-specific urinary casts.

- Coagulation complications: thrombocytopenia, endothelial injury or microvascular thrombosis.

- Neurological complications: altered mental status, irritability, decreased interaction, sleepiness or stupor.

- Vascular complications: decreased perfusion, bounding pulses, brisk capillary refill, low diastolic blood pressure and wide pulse pressure.

\subsection{Phylogenetic Overview}

Most septic patients (about 70\%) whose data was analysed in this thesis are respiratory cases. Most pulmonary cells express a large repertoire of genes under transcription control that are modulated by biomechanical forces and bacterial infections. Essential components of the innate immune system are the toll-like receptors (TLRs), which recognize not only microbial products but also degradation products released from damaged tissue providing signals that initiate 
inflammatory responses. Several different components are involved in TLR signalling, such as IL-1 receptor-associated kinases (IRAK), which results in the activation of pro-inflammatory cytokines, such as TNF- $\alpha$ and IL-6. Current evidence indicates that IRAK-3 (also known as IRAK-M) is a negative regulator of the TLR pathways and a master regulator of inflammatory processes during Sepsis $[8,9,10,11,12,13]$. This inflammatory mediated approach is a very active field of research both from a clinical and proteomics point of view. However, these IL approaches are still far from reaching widespread clinical practice.

Given that the genetic sequence of IRAK-3 is known for different species (most primates and rodents), it is possible to reconstruct the phyologenetic trees for these species $[14]^{1}$. Since the phylogenetic reconstruction by means of four different data analysis approaches (Unweighed Pair Group Method with Arithmetic Mean, Jukes-Cantor, Neighbour Joining and Maximum Likelihood -a good overview of these methods can be found in [14]) clearly groups the Homo Sapiens with the Macaque and Orangutan (see figure 2.1), it can be concluded that these three species shared a common ancestor with a similar IRAK-3 structure and, therefore, similar lung inflammation characteristics.

\subsection{Historic Overview}

From section 2.1, it can be concluded that Sepsis is at least as old as mankind. About 4,000 years ago, the Egyptians postulated that the intestine contained 2 a dangerous 'principle', which they defined as $\boldsymbol{W H} \boldsymbol{D H}$ and pronounced 'ukhedhu'. This principle could find its way into the vessels, settle anywhere in the body, or even 'rise to the heart' and kill [15].

The concept of WHDH makes sense, given that the intestines do, in fact, contain dangerous substances. From the Egyptians onward, auto-intoxication from the intestine has become a common explanation for certain pathologies. The fear of WHDH led the Egyptians to search substances that never suffer decay and, thus, may prevent it in wounds by means of sympathetic magic [16]. In fact, they devised some wound salves that were probably the best possible in those days. At the top of the list is honey, which is not only aseptic but also a powerful antiseptic.

Later on, in the $5^{\text {th }}$ century BC, the ancient Greeks adopted or reinvented the concept of auto-intoxication from the gut and elaborated on it. Our major sources of information are the Hippocratic books, where we find two words, which concern us: Sepsis $(\sigma \tilde{\eta} \psi \iota \varsigma)$ and pepsis $(\pi \tilde{\epsilon} \psi \iota \varsigma)$. Although these two words cannot be translated exactly, they represented two different forms of biological breakdown. Sepsis was very close to our concept of putrefaction and implied a bad smell, whereas pepsis was a composite of 'cooking', 'digestion', and 'fermentation'. Both can occur inside the body and, medically, pepsis was seen as helpful, whereas Sepsis was always dangerous. This later usage was also supported by Aristotle [17].

However, one has to wait until $c a .100 \mathrm{AD}$ to find the first documented case of Sepsis. Among the essays included in Plutarch's Morals (Vol. I Chapter XVI and Vol. III, Book VI) [18] is one entitled Precepts on Health, which is often

\footnotetext{
${ }^{1}$ Gene Data Source: http://www.ensembl.org/index.html

${ }^{2}$ Even though they could not see the intestinal flora by any optical means.
} 


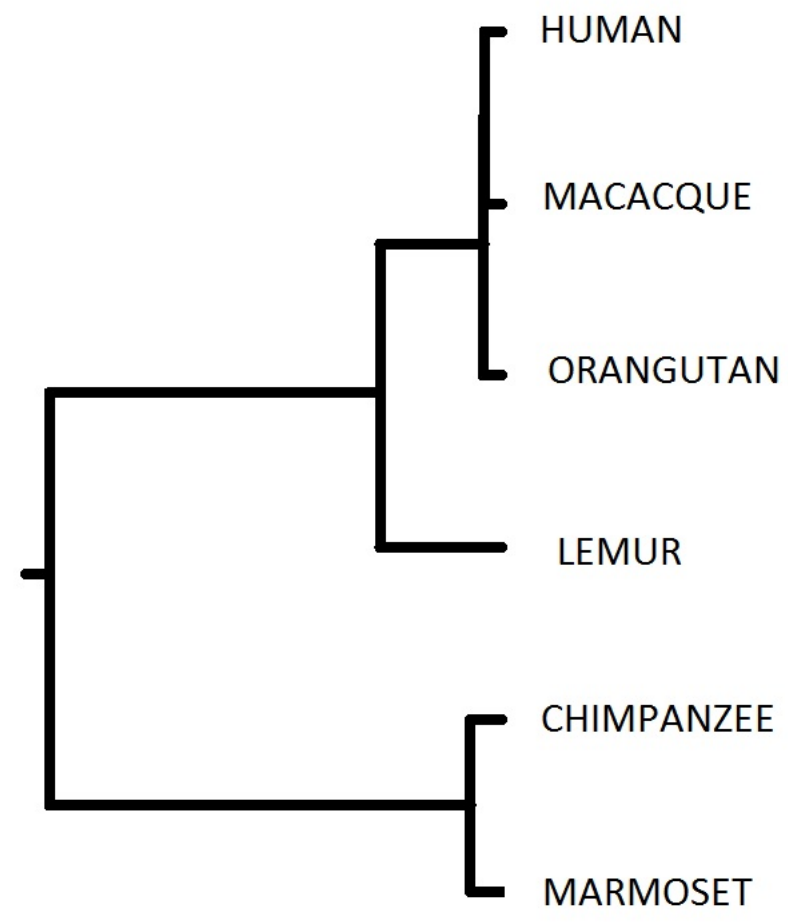

Figure 2.1: phylogenetic tree for the IRAK-3 Inflammation Toll Receptor 
cited by its Latin title De Tuenda Sanitate Praecepta. In Vol. I, Chapter XVI, we find the following story:

" [...] Niger, when he was teaching philosophy in Galatia, by chance swallowed the bone of a fish; but a stranger coming to teach in his place, Niger, fearing he might run away with his repute, continued to read his lectures, though the bone still stuck in his throat; from whence a great and hard inflammation arising, he, being unable to undergo the pain, permitted a deep incision to be made, by which wound the bone was taken out; but the wound growing worse, and rheum falling upon it [it became purulent] ${ }^{3}$, it killed him."

Beyond the remarkable surgical procedure [19], what is of interest to us is the fact that Niger's death was not due to the operation but due to the consequent infection. More particularly, what killed Niger was a post-surgical Sepsis, evidence of which manifested itself at the surgical site on which Plutarch's account is clear.

The concept of Sepsis presented above was used until the $19^{\text {th }}$ century and there are few pathophysiological investigations known during these centuries. In this regard, it is no surprise that the history of Sepsis is very much intertwined with that of surgical procedures, antiseptics (such as iodine) and drug discovery (the most outstanding being the discovery of antibiotics).

However, in the $17^{\text {th }}$ century, a doctor in Leyden named Herrman Boerhave postulated that toxic substances in the air were the cause for Sepsis. This theory was further expanded in the 19th century by Justus von Liebig who stated that it was the contact between wounds and oxygen that initiated the development of Sepsis.

During the second half of the $19^{\text {th }}$ century, an obstetrician at the Vienna General Hospital, Ignaz Semmelweis, took a revolutionary approach to preventing the death caused by puerperal fever. His department had an especially high mortality rate $(18 \%)$ and he discovered that it was common practice for students to examine pregnant women directly after pathology lessons. By that time hygienic measures such as hand washing or surgical gloves were not customary practice.

Semmelweis deducted that child bed fever was caused by "decomposed animal matter that entered the blood system" (recall the Egyptian principle outlined above). As a matter of fact, he succeeded in lowering the mortality rate to $2.5 \%$ by introducing hand washing with a chlorinated lime solution before every gynaecological examination. However, in spite of the clinical success, the hygienic measures were not accepted, and colleagues harassed him, being forced to leave the city. It took him until 1863 , more than 15 years after his findings, to publish his work "Aetiology, terminus and prophylaxis of puerperal fever" (Die Aetiologie, der Begriff und die Prophylaxis des Kindbettfiebers). The failure to achieve a professional reputation and the unrelenting opposition of the medical establishment may have facilitated the development of a psychiatric disease. Semmelweis was eventually committed to a lunatic asylum where he died from a wound infection probably as a result of the beatings he underwent there. It is an irony of fate that he died from a disease that he dedicated his life to fight. It was the surgeon Joseph Lister who managed to introduce the general procedure

\footnotetext{
${ }^{3}$ The words within brackets have been added for interpretation purposes.
} 
of instrument sterilization in medical practice. The methods initiated by Lister are not very different from those applied today.

Arguably, the most important breakthrough regarding Sepsis is due to the works of Louis Pasteur. Pasteur discovered that tiny cell organisms caused putrefaction and termed these organisms as bacteria (see definitions of Sepsis given below) and correctly deduced that these microbes could cause disease. He also made the significant discovery that bacteria in fluids could be killed by heating. This meant that a fluid could be sterilized.

At the beginning of the $20^{t h}$ century, the German physician H. Lennhartz initiated the change in the understanding of Sepsis from the ancient concept of putrefaction to the modern view of a bacterial disease. It was, however, his student Hugo Schottmüller (1867-1936), who in 1914 paved the way for a modern definition of Sepsis: "Sepsis is present if a focus has developed from which pathogenic bacteria, constantly or periodically, invade the blood stream in such a way that this causes subjective and objective symptoms". Thus, for the first time, the source of infection as a cause of Sepsis came into focus.

Although antiseptic procedures meant a huge medical breakthrough, it soon became apparent that a number of patients still developed Sepsis. In this preantibiotic time, the death rate was very high. These patients often showed very low blood pressure. This condition was called Septic Shock. Only with the introduction of antibiotics after WW II could the death rate of Sepsis be reduced further. With technological progress, intensive care medicine started to develop and Sepsis patients soon became the main patient fraction on ICUs [20].

\subsection{Clinical Overview}

\subsubsection{Definitions}

In August 1991, the American College of Chest Physicians/Society of Critical Care Medicine Consensus Conference took place with the goal of agreeing and standardizing a set of definitions to be applied to patients with Sepsis and its sequelae [21, 22], which is the reference mainly followed in this section. In this conference, new terms were proposed and others (like septicaemia) were abandoned from clinical practice. Broad definitions for Sepsis and SIRS were also proposed along with detailed physiologic parameters by which a patient could be categorized. Definitions for Severe Sepsis, Septic Shock, hypotension, and Multiple Organ Dysfunction Syndrome (MODS) were offered. These definitions have since been deployed and provided a good framework for the treatment of Sepsis. The aim of this subsection is to provide an overview of these definitions, which shall be used throughout this thesis. Figure 2.2 presents a summarized graph of the concepts outlined below.

\section{Systemic Inflammatory Response Syndrome, Sepsis and Septic Shock}

As stated above, Sepsis is defined as "the systemic response to infection". It is apparent that a similar, or even identical, response can arise in the absence of infection. Therefore, the term "Systemic Inflammatory Response Syndrome" $(S I R S)$ is proposed to describe this inflammatory process, independent of its cause. 


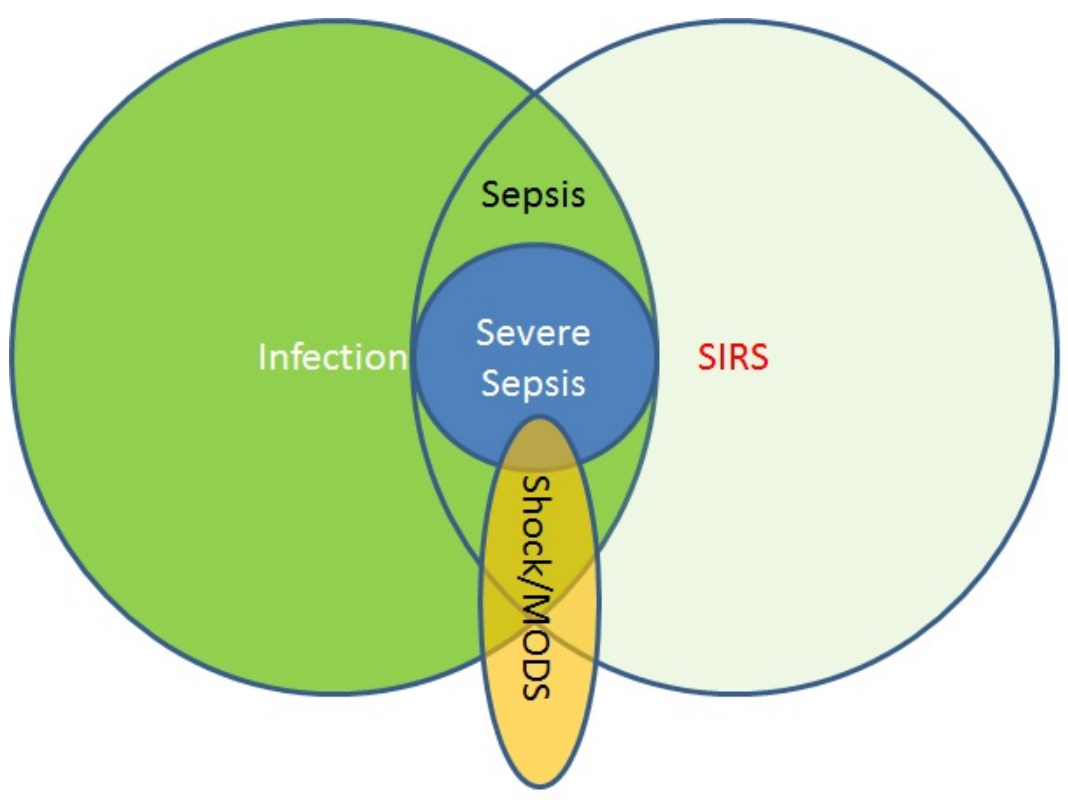

Figure 2.2: Sepsis Overview: The main sources of Sepsis is either an Infection or SIRS, after that it may evolve to Severe Sepsis, which in turn can evolve toward MODS or Septic Shock.

This Systemic Inflammatory Response can be seen following a wide variety of insults and includes, but is not limited to, more than one of the following clinical manifestations:

1. Body temperature higher than $38^{\circ} \mathrm{C}$ or lower than $36^{\circ} \mathrm{C}$.

2. Heart rate higher than 90 beats per minute (bpm).

3. Tachypnea, manifested by a respiratory rate higher than 20 breaths per minute or hyperventilation indicated by a $\mathrm{PaCO}_{2}$ of less than $32 \mathrm{mmHg}$.

4. Alteration in the white blood cell count, such as a count higher than $12,000 / \mathrm{cu} \mathrm{mm}$ or lower than $4,000 / \mathrm{cu} \mathrm{mm}$, or the presence of more than $10 \%$ immature neutrophils.

These physiological changes should represent an acute alteration from baseline in the absence of other known causes for such abnormalities, such as chemotherapy, induced neutropenia, and leukopenia.

The Systemic Inflammatory Response manifests itself in association with a large number of clinical conditions. Besides the infectious insults that may produce SIRS, non-infectious pathological causes may include pancreatitis, ischemia, multiple trauma and tissue injury, hemorrhagic Shock, immune-mediated organ injury, and the exogenous administration of the inflammatory process mediators such as tumour necrosis factor or other cytokines (see section 2.1).

A frequent complication of SIRS is the development of organ system dysfunction, including well-defined clinical conditions such as Acute Lung Injury (ALI), Shock, renal failure, and MODS. The term MODS is defined below. 
When SIRS is the result of a confirmed infectious process, it is termed Sepsis. In this clinical circumstance, the term Sepsis represents the Systemic Inflammatory Response to the presence of an infectious agent. In this regard, infection is defined as the microbial phenomenon characterized by an inflammatory response to the presence of micro-organisms or the invasion of normally sterile host tissue by those organisms. Bacteremia is the presence of viable bacteria in the blood stream. The presence of viruses, fungi, parasites, and other pathogens in the blood are described in a similar manner (i.e. viremia, fungemia, parasitemia).

Sepsis and its sequelae represent a continuum of clinical and pathophysiological severity. Of course, the degree of severity independently affects prognosis (as shall be investigated in this thesis). Some clinically recognizable stages of Sepsis include the following:

- Severe Sepsis: Sepsis associated with organ dysfunction, hypoperfusion abnormality, or Sepsis-induced hypotension. Hypoperfusion abnormalities include lactic acidosis, oliguria, and acute alteration of mental state.

- Sepsis Induced Hypotension: Presence of a systolic blood pressure of less than $90 \mathrm{mmHg}$ or a fall of $40 \mathrm{mmHg}$ or more from the baseline in the absence of other cause for hypotension (i.e. Cardiogenic Shock).

- Septic Shock: A subset of Severe Sepsis (i.e. it includes organ dysfunction and is therefore very closely related to MODS, as it shall be seen below), defined as Sepsis-induced hypotension and persisting despite adequate fluid resuscitation (fluid administration), along with the presence of hypoperfusion abnormalities or organ dysfunction. Patients receiving inotropic or vasopressor agents may no longer be hypotensive by the time they manifest hypoperfusion abnormalities or organ dysfunction. However, they would still be considered to suffer from Septic Shock.

\section{Multiple Organ Dysfunction Syndrome}

Multiple Organ Dysfunction Syndrome (MODS) is defined as the detection of altered organ function in the acutely ill patient. The term dysfunction identifies this process as a phenomenon in which organ function is not capable of maintaining homeostasis (system stability). This process, which may be absolute or relative, can be more readily identified as a continuum of change over time for which it must be considered that:

1. It describes a continuum of organ dysfunction, although specific descriptions of this continuous process are not currently available.

2. The recognition of early organ abnormalities must be improved so that treatment can be initiated at early stages in the evolution of the syndrome.

3. Changes in organ function over time can be viewed as an important element in its prognosis. When applied to MODS, existing measures of illness severity provide only a snapshot in time of this dynamic process, and are generally without reference to the natural course of disease.

4. It is subject to modulation by numerous factors at varying time periods, both interventional- and host-related. 
In the light of what has been said so far, MODS is understood to develop by two relatively distinct, but not mutually exclusive, pathways. Primary MODS is the direct result of a well-defined insult in which organ dysfunction occurs early and is directly attributable to the insult itself (for example, as the result of traumatic injury). In primary MODS, the participation of an abnormal and excessive host inflammatory response in both the onset and progression of the syndrome is not as evident as in secondary MODS.

Secondary MODS develops not as a result of the insult itself but, instead, as the consequence of a host response and is identified within the context of SIRS. SIRS is also a continuous process, and describes an abnormal host response that is characterized by a generalized activation of the inflammatory reaction in organs remote from the initial insult. Given that SIRS/Sepsis is a continuous process, MODS may be understood to represent the more severe end of the spectrum of severity of illness that characterizes SIRS/Sepsis. Therefore, secondary MODS usually evolves after a latent period following the inciting injury or event, and is most commonly seen to complicate severe infection.

\subsection{Systems for Scoring the Severity of Sepsis}

In normal clinical practice, and while treating the syndromes outlined in the previous section, clinicians are always trying to catch up with the pathology. In other words, they are treating severely ill patients at later stages of illness. It is also apparent that many of these patients who have more complex illnesses may be suffering from a combination of chronic and acute disease.

The rationale for using scoring systems in a clinical environment is to ensure that the increased complexity of disease in patients currently being treated is consistently represented for all those involved in the form of evaluations and descriptions. A specific goal of severity scoring systems is to use these important patient attributes to describe the relative risks of patients and identify where along the continuum of severity the patient resides. This should reduce the variability due to patient factors so that the incremental impact of new or existing therapies can be more precisely determined. Also, more precise measurements of patient risk should lead to new insights into disease processes and serve as a tool with which clinicians could more accurately monitor patients and implement the use of new therapies.

It is increasingly being recognized that the ultimate goal of severity scoring can be more than just obtaining a figure representing the degree of physiological disturbance. Severity scoring can be used in conjunction with other risk factors such as disease aetiology to anticipate and estimate outcomes such as ICU mortality. These estimates can be calculated at the time a patient presents for care or for entry into a clinical trial. Therefore, they can serve as a pretreatment protocol. They can also be updated during the course of therapy, thereby describing the course of illness and providing an alternative for the evaluation of response. What follows is a summary description of some of the scoring systems currently in use in medical procedure. 


\subsubsection{Sequential Organ Failure Assessment Score}

In 1994, the ESICM (European Society of Intensive Care Medicine) [2] organized a consensus meeting in Paris to create a so-called Sequential Organ Failure Assessment (SOFA) Score with the aim of objectively and quantitatively describing the degree of organ dysfunction/failure over time in groups of patients or even individuals. The main two major applications of the SOFA score are:

1. Improving the understanding of the natural history of organ dysfunction/failure and the interrelation between the failure of various organs / systems.

2. Assessing the effect of new therapies on the course of organ dysfunction/failure. This could be used to characterize patients at admission in the ICU (and even serve as an ICU entry criterion ${ }^{4}$ ), or to evaluate treatment efficacy.

Originally, the SOFA score was not designed to predict outcome but to describe a series of complications on the critically ill. Although any assessment of morbidity is related to mortality to some extent, the SOFA score was not designed just to describe organ dysfunction/failure according to mortality. However, and as investigated in this thesis, SOFA scores greater than 7 could present important ICU outcome prediction capabilities. Moreover, when combined with additional parameters, it provides a very powerful set of features not only for outcome assessment but also for the study of the evolution of Sepsis into its more severe states. The latter is one of the main design objectives of this particular score.

The SOFA limits the number of organs/systems under study to six, namely: Respiratory (inspiration air pressure), Coagulation (Platelet Count), Liver (Bilirrubine), Cardiovascular (Hypotension), Central Nervous System (Glasgow Coma Score), Renal (Creatinine or Urine Output). The scoring for each organ/system ranges from 0 for normal function to 4 for maximum failure/dysfunction. The final SOFA score is the addition of the dysfunction indexes for all organs/systems. Therefore, the maximum possible SOFA score is 24 , corresponding to maximum failure for all of the six organs/systems considered. Table 2.1 shows the SOFA Score calculation procedure.

In the light of what has been described so far and from a practical perspective, a SOFA score greater than 1 corresponds to Multiple Organ Dysfunction Syndrome (MODS), while Cardiovascular SOFA scores greater than 2 correspond to Septic Shock. Normally, SOFA scores are calculated at ICU admission. However, daily calculations of SOFA scores (Dynamic SOFA) [23, 24] provide valuable information about organ dysfunction evolution and prognosis. In our work, Dynamic SOFA was used to study the evolution of Septic Shock and the derivation of ICU prognostic indicators.

\subsubsection{Acute Physiology and Chronic Health Evaluation II}

"Acute Physiology and Chronic Health Evaluation II" (APACHE II) is a severityof-disease classification system [1]. After admission to an ICU, an integer score

\footnotetext{
${ }^{4}$ In this regard, during the 2010 flu pandemic in Australia, patients were admitted in the ICU with a maximum SOFA score of 7 .
} 


\begin{tabular}{|c|c|c|c|c|}
\hline SOFA Score Points & 1 & 2 & 3 & 4 \\
\hline $\begin{array}{c}\text { Respiration } \\
\mathrm{PaO}_{2} / \mathrm{FiO}_{2} \mathrm{mmHg}\end{array}$ & $<400$ & $<300$ & $<200$ & $<100$ \\
\hline $\begin{array}{c}\text { Coagulation } \\
\text { Platelet Count: Platelets } \times \frac{10^{3}}{\mathrm{~mm}^{3}}\end{array}$ & $<150$ & $<100$ & $<50$ & $<20$ \\
\hline $\begin{array}{c}\text { Liver } \\
\text { Bilirubine }[m g / d L]\end{array}$ & $1.2-1.9$ & $2.0-5.9$ & $6.0-11.9$ & $>12$ \\
\hline $\begin{array}{c}\text { Cardiovascular } \\
\text { Hypotension }\end{array}$ & $\mathrm{MAP}<70$ & $\begin{array}{c}\mathrm{DPM} \\
\text { or } \mathrm{DBT} \leq 5\end{array}$ & $\begin{array}{c}\mathrm{DPM}>5 \\
\mathrm{AD} \leq 0.1 \\
\mathrm{NAD} \leq 0.1\end{array}$ & $\begin{array}{c}\mathrm{DPM}>15 \\
\mathrm{AD}>0.1 \\
\mathrm{NAD}>0.1\end{array}$ \\
\hline $\begin{array}{l}\text { Central Nervous System } \\
\text { Glasgow Comma Score }\end{array}$ & $13-14$ & $10-12$ & $6-9$ & $<6$ \\
\hline $\begin{array}{c}\text { Renal } \\
\text { Creatinine }[m g / d L] \text { or } \\
\text { Urine Output }\end{array}$ & $1.2-1.9$ & $2.0-3.4$ & $\begin{array}{c}\quad 3.5-4.9 \\
\text { or }<500 \mathrm{ml} / \text { day }\end{array}$ & $\begin{array}{c}>5 \\
<200 \mathrm{ml} / \text { day }\end{array}$ \\
\hline
\end{tabular}

Table 2.1: SOFA Score table adapted from [2]. Here, MAP stands for Mean Arterial Pressure, DPM for dopamine, DBT for dobutamine, AD for adrenaline, and NAD for Noradrenaline. Dosages are given in $[\mu \mathrm{g} / \mathrm{Kg} \cdot \mathrm{min}]$.

from 0 to 71 is computed for the patient on the basis of several measurements. Higher scores imply a more severe disease and, therefore, a higher Risk of Death (ROD).

APACHE II was designed to measure the severity of disease for adult patients admitted to ICUs. The minimum age is not specified in the original study [1], but it is commonly recommended using APACHE II only for patients older than 15 years. This scoring system is applied in different ways:

- Some procedures are only carried out in, and some drugs are only prescribed to, patients with a given APACHE II score.

- The APACHE II score can be used to describe the morbidity of a patient when comparing their outcomes with that of other patients.

- Predicted mortalities are averaged for groups of patients in order to specify the group's morbidity.

Even though newer scoring systems have replaced APACHE II in some instances [25, 26], APACHE II continues to be used extensively in clinical practice, due to its simplicity of calculation and the abundance of related medical documentation.

The score is calculated from 12 routine physiological measurements (such as blood pressure, body temperature, heart rate, etc.) during the first 24 hours after admission (see figure 2.3), plus information about previous health status and some information obtained at admission (such as age). The resulting score should always be interpreted in relation to the illness of the patient. Once the initial score is determined within 24 hours of admission, no new score can be calculated during the ICU stay. If a patient is discharged from the ICU and 
readmitted, a new APACHE II score must be calculated. In this thesis, the APACHE II score was used to assess patient severity and also as a baseline measure for comparing ROD in Severe Sepsis. 


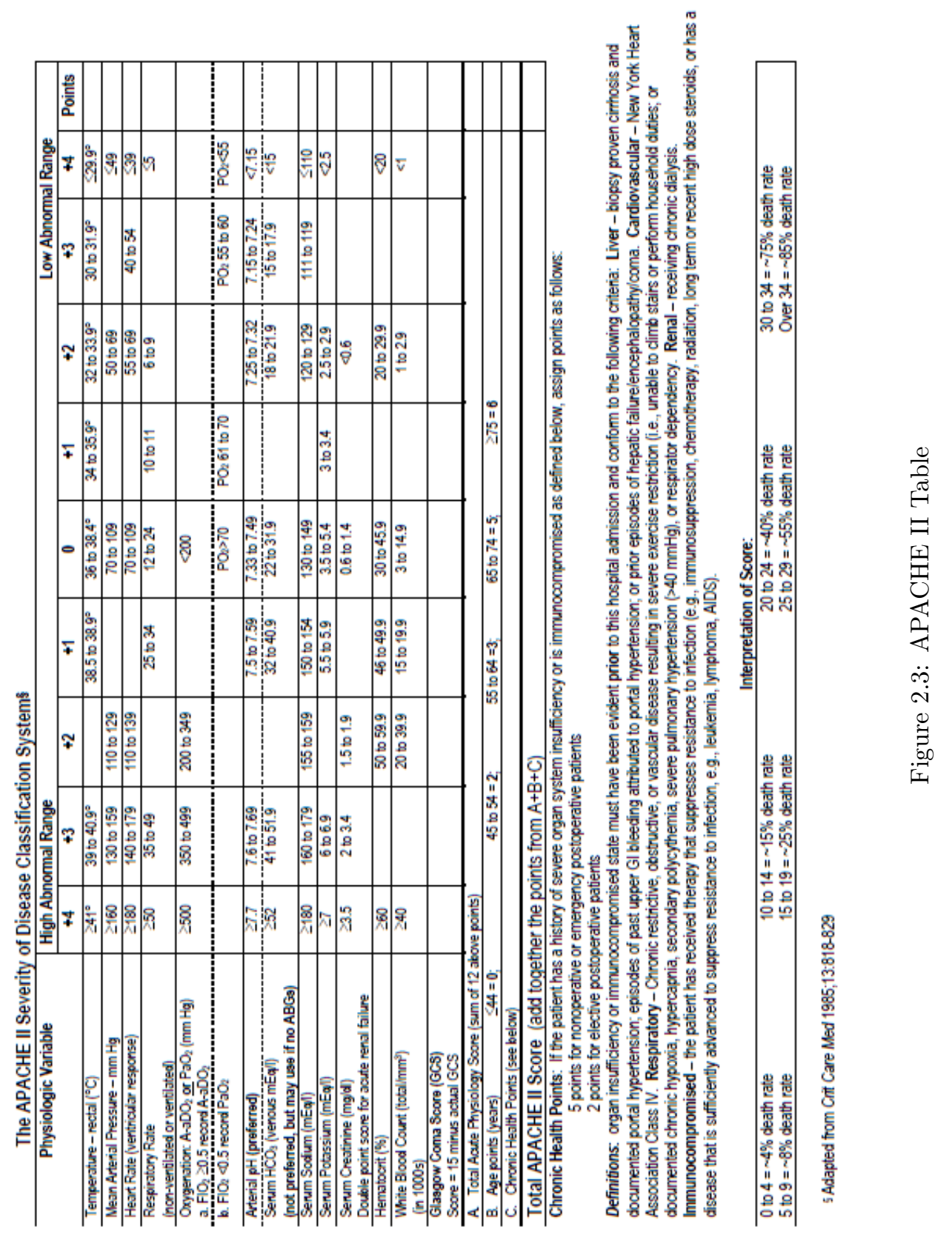




\section{Chapter 3}

\section{State of the Art: Quantitative Analysis of Sepsis}

No hay que empezar siempre por la noción primera de las cosas que se estudian, sino por aquello que puede facilitar el aprendizaje.

Aristotle

Current research in quantitative analysis of Sepsis using physiological measurements or standard scores is still at its very early stages. Different methodological approaches have been followed, with a diverse range of goals. Only a few studies have recently started to make use of quantitative machine learning and computational intelligence-related methods.

\subsection{Quantitative Analysis of the Pathophysiology of Sepsis}

Although the pathophysiology of Sepsis is fairly well understood by the medical community, the correlation between different clinical traits and the onset of Sepsis has not yet been studied in detail. For example, Arterial Resistance, Blood Flow, MAP and Reactive Hyperaemia and their relation to the severity of Sepsis are studied in [27], while, in [28], neuroautonomic modulation of heart rate and blood pressure were assessed in Sepsis or Septic Shock, concluding that:

\footnotetext{
"Uncoupling of the autonomic and cardiovascular systems occurs over both short- and long-range time scales during Sepsis, and the degree of uncoupling may help differentiate between Sepsis, Septic Shock, and recovery states."
}

Regarding the poor blood perfusion in tissue during Sepsis, a study by Ellis and colleagues [29] built a model with partial differential equations of the capillary network structure and oxygen transport from blood to tissue, and described how experimental values relate to model parameters. The reported 
simulations show the effects of Sepsis on oxygen transport heterogeneity and the development of tissue hypoxia.

In a different study, Ross and co-workers [30] derived a system of ordinary differential equations (modelled as a coupled system of three differential equations) together with an Artificial Neural Network (ANN) model of inflammation and Septic Shock. These equations take into consideration three main parameters (namely, pathogen influence, immunological response and cell damage), which are learned by means of an evolutionary approach (this approach is independent of the complexity of the objective functions) and, after that, four models are selected by minimum description length.

A Fuzzy Decision Support System (DSS) for the management of post-surgical cardiac intensive care unit (CICU) patients was described in [31]. The DSS encompasses an input module to evaluate the patient's hemodynamic status; a diagnostic module that implements the expert decision-making strategies; and a therapeutic module that incorporates a multiple-drug fuzzy control system for the execution of the therapeutic recommendations. The DSS is validated on a physiological model of the human cardiovascular hemodynamics whose parameters have been modified to reproduce the key pathological features of Sepsis.

Also in the field of the pathophysiology of Sepsis, it has been demonstrated that mitochondrial nitric oxide synthase (mtNOS) plays an important role in the onset of Septic Shock [32]. In turn, mtNOS is also related to ventricular contractility and, therefore, to the cardiovascular complications of Sepsis. Results suggest that mtNOS may contribute to the ventricular depression during Septic Shock.

There are also other inflammatory mediators during Septic Shock that may result in ischemia or other cardiovascular complications. In particular, Septic Shock has a direct impact in tissue perfusion and, therefore, in the most irrigated organs such as the stomach. In the light of this condition, the gastric mucosa, which can be monitored by means of gastric impedance spectroscopy, will deteriorate during a Septic Shock prior to MODS or ischemia, as investigated in [33] and [34].

In addition to the articles described above, [35] presents an architecture for multi-dimensional temporal abstraction and its application in Pediatric Intensive Care Units (PICU). According to the authors, "temporal abstraction (TA) provides the means to instil domain knowledge into data analysis processes and allows transformation of low level numeric data to high level qualitative narratives. TA mechanisms have been primarily applied to uni-dimensional data sources equating to single patients in the clinical context". This architecture enables the analysis of data arriving from a number of patients, as well as the detection of several conditions within the PICU, including Sepsis.

Different papers in this field address the problem of rule generation [36] [37]. It is argued in [36] that, due to the irregularities in patient data recording at ICUs, it is worth exploring a generalization paradigm (i.e., individual cases generalized to more general rules) rather than an association paradigm, which combines single data attributes from an individual patient. The algorithm for rule generation and classification presented in this work is based on heuristically generated set-based data intersections in the development of Sepsis. On the other hand, the approach in [37] entails embedding a rule generation algorithm into a medical data mining cycle. The architecture of the system is improved 
by means of a growing trapezoidal basis function network.

Beyond [37], there are other studies that deploy ANNs for the study of Sepsis. Amongst them, [38] presented a clinical study examining SIRS and MODS in the ICU after cardiac and thoracic surgery. The ANN-based prediction system introduced in this work takes into consideration the time interval between the onset of Sepsis and until the receding of the symptoms. Then, from this set of observed data, an ANN that predicts the evolution of Sepsis into Severe Sepsis is built. One of the main findings of this study is that there is a significant correlation between the number of SIRS episodes and the outcome of Severe Sepsis for each individual patient.

The initiatives related to the application of ANNs to the study of Sepsis have also resulted in expert systems such as the one called SES, described in [39], which was designed for the diagnosis of pathogens and prescription of antibiotics. The performance of SES has been evaluated in [40] and improvements based on the available knowledge-base clinical database have been proposed.

Support Vector Machines (SVM) have also been used for the prediction of Sepsis. Kim et al. [41] applied them to study Sepsis in post-operative patients. More specifically, they applied SVMs for regression and One-Class SVM for studying the temporal evolution of Sepsis using data from 1,239 patients, reporting an AUC of $94 \%$ for the detection/prediction of Sepsis. This method has also been used for the diagnosis of Sepsis. Wang et al. [42] built a DSS for the diagnosis of Sepsis based on the following attributes: Age, Heart Rate, Body Temperature, Respiration Rate, White Cell count and the APACHE II score. This study reported an AUC of $88 \%$, a sensitivity of $87 \%$, and a specificity of $88 \%$.

\subsection{Quantitative Analysis of the Prognosis of Sep- sis}

The SIRS pathology is known to be a quite sensitive indicator of Sepsis [43], but also one of poor specificity. Different studies have shown that the incidence of SIRS is quite high in critical patients in general. For example, Pittet et al. [44] presented a SIRS incidence of up to $93 \%$ in critical care patients, while Rangel et al. showed an incidence of $68 \%$ [43]. The latter study also shows that $25 \%$ of patients with SIRS developed a Sepsis, $18 \%$ presented Severe Sepsis, and $4 \%$ of them, Septic Shock. Regardless of these incidence ratios, the early detection of patients with a higher ROD remains a challenge.

The MEDS (Mortality in Emergency Department Sepsis) score is a collection of variables routinely recorded in the emergency departments (terminal illness, tachypnea/hypoxaemia, Septic Shock, platelet count, age, lower respiration infection, bands, nursing home resident and mental status). It was shown in [45] to yield an AUC of 0.88 for the population under study: patients at the emergency department with SIRS (not taking into account those septic patients admitted in the emergency department who were not critical enough to be admitted in the ICU).

Since the publication in 1985 of the Organ System Failure (OSF) score by Knaus [46], which is a prognosis scale to evaluate and quantify MODS, alternative prognostic scores have been developed. They include the already reviewed 
APACHE II score [1], as well as the SOFA score [2], and the LODS (Logistic Organ Dysfunction System) [47]. Two prognostic scores based on the PIRO model (predisposition, insult/infection, response and organ dysfunction) have also been recently proposed: the SAPS3 PIRO score ([48]: AUC 0.77) and the PIRO score ([49]: AUC 0.70).

Machine learning methods have been used with varying success for the prediction of mortality caused by Sepsis. A diagnostic system for Septic Shock based on ANNs (Radial Basis Functions -RBF- and supervised Growing Neural Gas) was presented in [50], reporting an overall correct classification rate of $67.84 \%$, with a high specificity of $91.61 \%$, but an extremely poor sensitivity of $24.94 \%$. Also in this area, Brause et al. [51] applied an evolutionary algorithm to an RBF network (the MEDAN Project) to obtain, over a retrospective dataset, a set of predictive attributes for assessing mortality for Abdominal Sepsis, namely Systolic and Diastolic blood pressure and thrombocytes. This study reported an AUC of 0.90-0.92.

SVM methods have also been used in this context. Tang et al. [52] presented a SVM-based system for Sepsis and SIRS prediction from non-invasive cardiovascular spectrum analysis, reporting an overall accuracy of $84.62 \%$, with a rather low specificity of $62.50 \%$ and a high sensitivity of $94.44 \%$.

As described in previous sections, Sepsis can evolve into more critical conditions (namely, Severe Sepsis and Septic Shock) and it can also result in the death of the patient (60\% for Septic Shock). Medical symptoms were modelled in [53] as observations caused by the transitions in time in a Hidden Markov Model (HMM), where each patient class (surviving or not) defines its own transition probabilities between the states, especially to the death and dismissal state. Therefore, at least two HMM models are derived: one for the surviving patients and one for deceased. The diagnostic approach presented in this paper consists of presenting the patient data to a system which computes the probability for them to be either part of the surviving or the non-surviving HMM. According to authors, the understanding of the underlying state transition probabilities results in a "prediction probability success of about 91\%". This study goes beyond the clinical septic evolution described above and considers the different evolution states during an episode of Septic Shock.

A predictor based on the physiological data available from the IMPACT project $^{1}$ was defined in [54]. It studies the correlations between HR, MAP, Body Temperature and Respiration Rate, in order to distinguish between critically ill adult patients with and without Sepsis in the first 24 hours of admission to an ICU. This study concludes that MAP and Body Temperature are independently related to the onset of Sepsis. However, this clinical viewpoint is more related to the cardiovascular function and it is therefore more predictive of Severe Sepsis and Septic Shock.

Also regarding HR monitoring, HR variability was studied in [55], and a predictive model based on this parameter was developed in search of abnormal HR characteristics (HRC) prior to neonatal Sepsis. The predictive model developed in this article is based on multivariate logistic regression models adjusted for repeated measures, with the HRC values as predictor variables prior to the deterioration on the condition of the newborn (i.e., CRASH: Cultures, Resuscitation and Antibiotics Started Here). This article concludes that real-time

\footnotetext{
${ }^{1}$ www.piccm.com
} 
monitoring of HRC may result in early diagnosis and treatment of neonatal Sepsis.

\subsection{Limitations of Existing Quantitative Analysis}

Sepsis is a clinical syndrome that can only be diagnosed a posteriori by the concurrence of several clinical signs, as described in Chapter 2. This of course imposes a great limitation to the different systems and approaches currently used for ascertaining the presence of Sepsis. Despite this limitation, there is still room for testing different clinical traits or even co-ocurrent factors that may have an impact in the presence or prognosis of sepsis, which are not routinely measured. It also believed that the application of Machine Learning techniques may help in shedding some light on some open debates in the clinical practice. For instance, one question that still lingers in the clinical literature is should we stop or continue statins treatment during sepsis?. This is just but one open problem/limitation to treatment that needs to be addressed.

Regarding the prognosis of Sepsis, and to the best of our knowledge, the best one could do is to perform haemocultures and administer antibiotics during the very first hours of evolution. Time of treatment is of paramount importance. In this regard, one of the main limitations encountered is that the most widely used indicators in clinical practice like the APACHE II ${ }^{2}$ lack specificity despite having an acceptable sensitivitity ( 0.82 sensitivity and 0.55 specificity). This same specificity problem is found for the indicator tailored for Sepsis, namely SAPS, with a sensitivity and specificity of 0.69. Finally, the indicator SOFA is only related to organ failure and, therefore, does not provide ROD. However, it is widely accepted that SOFA scores greater than 7 are associated with higher mortality rates. This fact is also studied in this thesis.

Over the last years, the Lilly pharamaceutical company has been studying a new treatment for named Xigris ${ }^{\mathrm{TM}}$ (see, for example [56] and [6]), which is a recombinant of the human activated $\mathrm{C}$ protein. This protein clearly plays a role in the inflammatory cascade and has become the first drug approved by the U.S. Food and Drug Administration (FDA) and the European Agency for the Evaluation of Medicinal Products for treatment of patients with Severe Sepsis. Given the risks of this treatment, it has been approved for use in patients with a high ROD ascertained, for example, by means of the APACHE II score [56]. Not only does this impose a further risk for patients detected as a false positive (leading to low specificity) but also to the National Health Systems as a whole due to the elevated costs of treatment (about $30.000 \mathrm{USD} /$ day $^{3}$ ). There is a clear need for timely detection of Sepsis (according to the PROWESS studies [6], Xigris only works during the first hours of evolution) and also improving specificity and sensitivity of the indicators available.

Some improvement has already been detected for given patient populations (see [51] above), which presents an AUC of 0.90 for abdominal sepsis. Unfortunately, this is one of the most easily detected forms of Sepsis, since it takes place right after surgery in most of the cases, with clear symptoms (fever after surgery). Therefore, most of the approaches analysed are either limited in terms of patient base or base pathology (i.e. they only look at a certain stage of Sepsis

\footnotetext{
${ }^{2}$ this indicator was been designed for assessing the ROD in the ICU and not just Sepsis

${ }^{3}$ private conversation with Prof. Dr. Roger Mark, from MIT
} 
like Shock or MODS). There are also limitations in terms of the study design: prospective vs. retrospective. The latter being the most easily implemented, but also the most disputable when it comes to the results. Regarding the variables or clinical factors involved, often little attention is paid to the clinical eye (for example, decrease of SOFA score and extubation or decrease of lactate levels are clear signs of good prognosis), while other variables are overlooked. We do not advocate to follow this instinct blindfolded, but just put it to a test for confirmation. It is also believed that the complexity of the syndrome at hand calls for a more "generative" approach to ascertain the prognosis of Sepsis by means of a set of attributes that give a clear context of the patient at a given time. 


\section{Chapter 4}

\section{Background: Algebraic Statistical Models, Algebraic Exponential Families and Generative Kernels}

And now to something completely different.

Monty Python

The aim of this chapter is to present the necessary mathematical framework for the latter study of Sepsis by means of Algebraic Statistical Models in general and the marginal dependence between variables in particular. It is this marginal dependence study that shall be used later to derive the underlying relations and Graphical Models by means of the Hammersley Clifford theorem. The mathematical background presented in this chapter is used in chapters 6 and 8 . These chapters include the study the incidence of Sepsis in the geographic area covered by the Vall d'Hebron University Hospital in Barcelona, Spain. This incidence is modeled as a hidden variable in a graphical model. We also use the mathematical approach presented in this chapter to derive a new generative kernel to study the prognosis of Severe Sepsis. The main contributions of this section are the Quotient Basis Kernel obtained from Gröbner bases, the simplified Fisher kernel and the representation the kernels based on the Jensen-Shannon metric in an algebraic context.

\subsection{Polynomial Representation: Outline in Three Examples}

The aim of the following three examples is to intuitively introduce the algebraic background that shall be formally described throughout this chapter and to provide the main ideas that shall be used throughout this $\mathrm{PhD}$ thesis. The first example provides the first (and most obvious) layer of algebraization, where 
linear regression models in polynomial form are presented. This can be further generalized to polynomial regression. In this regard, spline regression may also be presented algebraically.

The second example is the most technical in the sense that not only does it introduce the basics of interpolative polynomials, but also one of the main issues that must be addressed in this thesis: that polynomial residuals on high dimensions are not unique. The only way we have to guarantee uniqueness for the expressions of our interpolation polynomials is through Algebraic Geometry.

Finally, it is this Algebraic Geometry machinery that will allow us to step into the most abstract level of algebraization. The third example provides a simple presentation of this level of abstraction where exponential family distributions are treated as polynomials in parameter space (sometimes this is also done in sample space) so that the algebraic description presented in this chapter can used for this particular set of probability density distributions.

\subsubsection{Linear and Polynomial Regression}

In a general classification/regression problem, we are interested in obtaining a response $y$ from an input $x$. Let $\Psi=\left(X_{1}, \cdots, X_{p}\right)$ be the matrix of inputs. therefore

$$
y=\omega^{t} \Psi
$$

where $\Psi$ takes different forms depending on the problem/model at hand. For example, if we have $N$ points $x_{i}: i \in\{1, \ldots, N\}$ of dimension $p$, an ordinary least square regression problem, $\omega$ takes the form:

$$
\omega=\left(\Psi^{t} \Psi\right)^{-1} \Psi^{t} y
$$

where $\Psi$ is the $N \times p$ observations matrix. Our ability to estimate the parameter vector $\omega$ under standard theory is equated with: $\Psi$ is $N \times p$ full rank or $\operatorname{Rank}(\Psi)=p<N$ where $\omega$ is a p-dimensional vector and $N$ is the number of design points. In another example, the one-dimensional polynomial regression

$$
y(x)=\sum_{j=0}^{p-1} \omega_{j} x^{j}
$$

needs $p$ independent design points ${ }^{1}$ so that the matrix $\Psi=\left(X_{1}, \cdots, X_{p}\right)$ has full rank. Also for submodels with fewer than $p$ terms, the $\Psi$ matrix has full rank.

\subsubsection{Interpolation}

Imagine that we observe three distinct points $\left(a_{i}, y_{i}\right): i \in 1, \ldots, 3$ in a supervised learning experiment. It is easy to show that there is a unique quadratic curve through these points [57]. Let us define the polynomial

$$
d(x)=\left(x-a_{1}\right)\left(x-a_{2}\right)\left(x-a_{3}\right)
$$

\footnotetext{
${ }^{1}$ Intuitively these points are equivalent to design points in Experimental Design. These p-dimensional points also live in the support of the underlying probability distribution.
} 
whose zeros are the observed/support points. Any other polynomial $p(x)$ running through the support points also fulfils $p\left(x_{i}\right)=y_{i}$ (for $\left.i=1,2,3\right)$. Without loss of generality we can write

$$
p(x)=s(x) d(x)+r(x),
$$

where $r(x)$ is the remainder when $p(x)$ is divided by $d(x)$. Since, by construction, $d(x)$ has $a_{i}$ as roots, it is obvious from the equation above that

$$
y_{i}=p\left(a_{i}\right)=r\left(a_{i}\right),(i=1,2,3) .
$$

By construction, our polynomial $p$ can be interpreted as an interpolation function with value $y_{i}$ at the point $a_{i}$ or, also, as the function defined only on the support points and again with value $y_{i}$ at $a_{i}$ for $(i=1,2,3)$. However, a word of caution must be given should we use this argument in high dimensions $(>2)$ since the division operation and the remainder themselves are not unique [57]. For this reason, we need to move into the field of Algebraic Geometry in order to guarantee unique representations. This shall be done through the definitions and theorems: term ordering, varieties, polynomial ideals, the Hilbert Basis theorem and, finally, Gröbner bases.

\subsubsection{Polynomial Representation of a Univariate Gaussian Variable}

In this third example, we show a more profound level of algebraization that will be used throughout this thesis. Let $X$ be a Bernoulli variable taking values in the support $\{0,1\}$ with probability $q$. By the central limit theorem, after $n$ repetitions with $n$ sufficiently large the sum of Bernoulli variables converge to $N(\mu=n q, \sigma=n q(1-q))$, the raw interpolator of the logarithm for this variable takes the form:

$$
p(x)=-\left(\log \left(\frac{2 \pi}{\sigma}\right)+\frac{\mu^{2}}{2 \sigma^{2}}\right)+\frac{\mu}{\sigma^{2}} x-\frac{1}{2 \sigma^{2}} x^{2} .
$$

The interpolator after exponentiation is

$$
\hat{p}(x)=\exp \left\{-\left(\log \left(\frac{2 \pi}{\sigma}\right)+\frac{\mu^{2}}{2 \sigma^{2}}\right)+\frac{\mu}{\sigma^{2}} x-\frac{1}{2 \sigma^{2}} x^{2}\right\} .
$$

Defining $\phi(\eta)=-\left(\log \left(\frac{2 \pi}{\sigma}\right)+\frac{\mu^{2}}{2 \sigma^{2}}\right), \eta_{1}=\frac{\mu}{\sigma^{2}}$ and $\eta_{2}=\frac{-1}{2 \sigma^{2}}$. Setting $\zeta_{0}=$ $e^{\phi(\eta)}, \zeta_{1}=e^{\eta_{1}}$ and $\zeta_{2}=e^{\eta_{2}}$ and noticing that the support of our Bernoulli distribution takes values on an integer grid, we have the representation

$$
\hat{p}(x)=\zeta_{0} \zeta_{1}^{x} \zeta_{2}^{x^{2}} .
$$

This coincides with the form of the regular exponential family for a univariate Gaussian

$$
p(x)=\exp \left\{\eta^{t} T(x)-\phi(\eta)\right\}
$$

where $T(x)$ is the vector with components $x$ and $x^{2}$. Later on we will see that $T(x)$ correspond to the sufficient statistics of a Regular Exponential Family. 
These sufficient statistics shall be used as building blocks for our Generative Kernels. The example shown here is very powerful in the sense that sets the intuitive basis for the implicit representation of Regular Exponential Families in the ring of polynomials. This result will be used to algebraically derive the generative kernels using the sufficient statistics of the Regular Exponential Family as the principal building block.

By now we should have noticed the deep interplay between different parametrizations. In the next sections it will also become apparent that another parametrization is needed in terms of moments. These parametrizations become even harder because statistical models or submodels are obtained by imposing restrictions on the parameters. In this thesis we will define an Algebraic Statistical Model $(A S M)$ as one which adopts one of these parametrizations and for which the restrictions on the parameters themselves are also polynomial [57]. A more formal definition of these ASM shall be given below. An important example of these models are independence models, which force factorization of the raw polynomial interpolators in parameter space and map additivity inside the exponential representation and factorization in the $\zeta$. Conditional independence models as used in this $\mathrm{PhD}$. are also examples of ASM.

\subsection{Algebraic Models}

In this section we present the definition of Algebraic Models as given in [57] where factors or inputs are denoted by $x$, responses or outputs are denoted by $y$, parametric functions denoted by $\eta$ or functions of $\eta$. These are related by polynomial algebraic relations, possibly implicit. Another feature of this definition is that constraints of polynomial type can be included in the specification of the model. Implicit models and the introduction of constraints can lead to the use of dummy variables.

The parameters of the model as interpreted in statistics are functions of any form with the restriction that they belong to a specified field. For example $\mathbb{Q}\left(\eta_{1}, \ldots, \eta_{p}\right)$ is the set of all rational functions in $\eta_{1}, \ldots, \eta_{p}$ with rational coefficients. Another example is $\mathbb{Q}\left(e_{1}^{\eta}, \ldots, e_{p}^{\eta}\right)$ the set of all exponential rational functions. Parameters are treated as unknown quantities and in most cases appear in linear form. The algebraic space used is the commutative ring of all polynomials $\mathbb{K}\left[x_{1}, \ldots, x_{s}\right]$ in the indeterminates $x_{1}, \ldots, x_{s}$ and with coefficients in the field $\mathbb{K}$.

Definition 1. [57] An initial ordering is a total order on the indeterminates $x_{1}, \ldots, x_{s}$.

When the indeterminates are indexed from 1 to $s$ such as $x_{1}, \ldots, x_{s}$ it is convention to consider an initial ordering $x_{i} \succ x_{i+1} \forall i=1 \ldots s-1$.

Definition 2. [57] The quantities of the form $x_{1}^{\alpha_{1}}, \ldots, x_{s}^{\alpha_{s}}$ with $\alpha_{i} \in \mathbb{Z}_{+} \forall i=$ $1, \ldots, s$ are called terms.

Definition 3. [57] The set of all terms in $s$ indeterminates is denoted by $\operatorname{Term}\{s\}$.

For a given initial ordering a term is specified by the vector of length $s$ of its exponents. Therefore $\operatorname{Term}\{s\}$ is coded by $\mathbb{Z}_{+}^{s}$ 
Definition 4. [57] Term Ordering

A term-ordering on $\mathbb{K}[x]$ is an ordering relation $\succ_{\tau}$ (or $\tau$ or $\succ$ ) on $\operatorname{Term}\{s\}$, that is the terms of $\mathbb{K}[x]$ satisfying

1. $x^{\alpha} \succ 1 \forall x^{\alpha}$ with $\alpha \neq 0$ and

2. $\forall \alpha, \beta, \gamma \in \mathbb{Z}_{+}^{s}$ such that $x^{\alpha} \succ x^{\beta}$, then $x^{\alpha} x^{\gamma} \succ x^{\beta} x^{\gamma}$

Definition 5. [57] Let $x_{1}, \ldots, x_{s}$ be indeterminates and let the initial ordering be $x_{i} \succ x_{i+1} \forall i=1 \ldots s-1$. The log operator is the function

$$
\begin{gathered}
\log : \operatorname{Term}\{s\} \rightarrow \mathbb{Z}_{+}^{s} \\
x^{\alpha}=\left(x_{1}^{\alpha_{1}}, \ldots, x_{s}^{\alpha_{s}}\right) \mapsto\left(\alpha_{1}, \ldots, \alpha_{s}\right)
\end{gathered}
$$

For example, a valid term ordering for the polynomial $f=-1 / 50 x y z+$ $3 / 100 x y+9 / 100 x z-3 / 25 y z-21 / 100 x+27 / 100 y+8 / 25 z+7 / 25$ is $x y z \succ x y \succ$ $x z \succ x \succ y z \succ y \succ z \succ 1$. This polynomial is the interpolation polynomial of the support points for our study on statins presented in this PhD Thesis. Another example of term ordering for another polynomial would be $x^{4} y^{7} \succ x^{4} y$.

Definition 6. [57] Let $\tau$ be a term-ordering on $\mathbb{K}[x]$ and $f$ a polynomial in $\mathbb{K}[x]$. The leading term of $f, L T_{\tau}(f)$ is the largest term with respect to $\tau$ among the terms in $f$. The leading coefficient $L C_{\tau}(f)$ is the coefficient of $L T_{\tau}(f)$. The leading monomial $L M_{\tau}(f)$ is the product $L C_{\tau}(f) L T_{\tau}(f)$.

For example, in our interpolation polynomial, the leading term $\operatorname{LT}_{\tau}(f)$ is $x y z$, the leading coefficient is $\mathrm{LC}_{\tau}(f)-1 / 50$ and the leading monomial $\mathrm{LM}_{\tau}(f)$ is $-1 / 50 x y z$.

Definition 7. [58] Monomials

A monomial in indeterminates $t_{1}, \ldots, t_{n}$ is a formal expression of the form $t^{\beta}=t_{1}^{\beta_{1}} t_{2}^{\beta_{2}} \cdots t_{n}^{\beta_{n}}$, where $\beta=\left(\beta_{1}, \ldots, \beta_{n}\right)$ is the non-negative integer vector of exponents.

Definition 8. [58] Polynomials

A polynomial $f=\sum_{\beta \in B} c_{\beta} t^{\beta}$ is a linear combination of monomials where the coefficients $c_{\beta}$ are in a fixed field $\mathbb{K}$ and $B \subset \mathbb{Z}_{+}^{n}$ is a finite set of exponent vectors. The collection of all polynomials in the indeterminates $t_{1}, \ldots, t_{n}$ with coefficients in a fixed field $\mathbb{K}$ is the set $\mathbb{K}[\mathbf{t}]=\mathbb{K}\left[t_{1}, \ldots, t_{n}\right]$. The collection of polynomials $\mathbb{K}[\mathbf{t}]$ has the algebraic structure of a ring. Each polynomial in $\mathbb{K}[\mathbf{t}]$ is a formal linear combination of monomials, that can also be considered as a function $f: \mathbb{K}^{\ltimes} \rightarrow \mathbb{K}$, defined by evaluation. Throughout this thesis we will focus on the ring $\mathbb{R}[\mathbf{x}]$ of polynomials with real coefficients.

The notion of ordering and term-ordering is of capital importance to guarantee the uniqueness of our basis representations, interpolations and studies in conditional independence.

Definition 9. [57] Variety

The algebraic variety of the finite set of polynomials $f_{1}, \ldots, f_{r}$ in $\mathbb{K}\left[t_{1}, \ldots, t_{n}\right]$ is the set

$$
\operatorname{Variety}\left(f_{1}, \ldots, f_{r}\right)=\left\{\left(a_{1}, \ldots, a_{n}\right) \in \mathbb{K}^{n}: f_{j}\left(a_{1}, \ldots, a_{n}\right)=0, j=1, \ldots, r\right\}
$$


Definition 10. [57, 58] Algebraic Model

Let $\mathbb{K}$ be a field, called the field of constants. Let $\mathcal{K}$ be a field of functions $\phi: \eta \rightarrow \mathbb{K}$, with $\eta$ the set of parameters; $\mathcal{K}$ is called the field of parametric functions. Let $x=\left(x_{1}, \ldots, x_{d}\right)$ be the control factors, $y=\left(y_{1}, \ldots, y_{p}\right)$ be the responses and $t=\left(t_{1}, \ldots, t_{h}\right)$ be the dummy variables. An algebraic model is a finite list of polynomials $f_{1}, \ldots, f_{q}, h_{1}, \ldots, h_{l}$ such that $f_{i} \in \mathcal{K}[x, y, t]$ and $h_{j} \in \mathbb{K}[x, t]$. The variety $\operatorname{Variety}\left(f_{i}, h_{j}: i=1, \ldots, q ; j=1, \ldots, l\right) \in \mathcal{K}^{d+p+h}$ is called the model variety and the variety $\operatorname{Variety}\left(h_{j}\right) \in \mathbb{K}^{d+h}$ is called the input variety.

Definition 11. Algebraic Statistical Model

A statistical model that can be specified by means of a variety

$$
\operatorname{Variety}\left(f_{1} \cdots f_{q}, h_{1} \cdots h_{l}\right) \in \mathcal{K}^{d+p+h}
$$

with respect to a set of parameters (with the ideal denoted by IdealVariety) is an Algebraic Statistical Model.

Definition 12. Polynomial Ideal:

1. A polynomial ideal $I$ is a subset of a polynomial ring $\mathbb{K}[x]$ closed under sum and product by elements of $\mathbb{K}[x]$. Specifically the set $I \subset \mathbb{K}$ is an ideal if $\forall f, g \in I$ and $s \in \mathbb{K}$ the polynomials $f+g$ and sf are in $I$.

2. Let $F$ be a set of polynomials. The ideal generated by $F$ is the smallest ideal containing $F$. It is denoted $\langle F\rangle$.

3. An ideal $I$ is radical if $f \in I$ whenever a positive integer $m$ exists such that $f^{m} \in I$.

4. The radical of an ideal $I$ is the radical ideal defined as $\sqrt{I}=\left\{f \in \mathbb{K}: \exists m \mid f^{m} \in I\right\}$

Definition 13. An ideal $I$ is finitely generated if there exist $f_{1}, \ldots, f_{r}$ polynomials in $\mathbb{K}[x]$ such that for any $f \in I$ there exist $s_{1}, \ldots, s_{r}$ polynomials of $\mathbb{K}[x]$ such that

$$
f=\sum_{i=1}^{r} s_{i} f_{i} .
$$

We write $I=\left\langle f_{1}, \ldots, f_{r}\right\rangle$ and the set $\left\{f_{1}, \ldots, f_{r}\right\}$ is called a basis of $I$.

Theorem 1. [57] Hilbert Basis Theorem

Every ideal in $\mathbb{K}[x]$ has a finite basis.

\subsubsection{Division}

The operations over $\mathbb{K}[x]$ are sum, products (with scalars and other polynomials) and polynomial division. It is also of particular importance the simplification of monomial fractions. Polynomial division may not be unique and requires the notion of term-ordering as presented above. The following theorem summarizes the division algorithm for univariate polynomials.

Theorem 2. [57] For every pair of polynomials, $f$ and $g$ in one indeterminate, there exist unique polynomials $s_{g}, r$ such that $L T(g) \succ L T(r)$ and $f=s_{g} g+$ $r$, where the leading terms are with respect to the only term ordering in one dimension. The division algorithm returns $s_{g}$ and $r$. 
In more dimensions the situation is less straightforward.

Theorem 3. [57] Let $f, g_{1}, \ldots, g_{t}$ be in $\mathbb{K}[x]$ and $\tau$ a term-ordering. There exist $s_{1}, \ldots, s_{t} \in \mathbb{K}[x]$ and $r \in \mathbb{K}[x]$ such that

$$
f=\sum_{i=1}^{t} s_{i} g_{i}+r
$$

and $L T_{\tau}(r)$ is not divisible by any of the $L T_{\tau}\left(g_{i}\right)$

\subsubsection{Gröbner Bases}

The Hilbert basis theorem 1 provides a very powerful result since it states that any ideal is finitely generated (even if the generating set is not necessarily unique). Another powerful result [57] is that this generation basis is of a special type called Gröbner Basis, which we define below. These bases will become essential in the derivation of regression/interpolation polynomials and also for the algebraic derivation of the Fisher and Quotient Basis Kernels.

Definition 14. [57] Gröbner Basis

Let $\tau$ be a term ordering on $\mathbb{K}[x]$. A subset $G=g_{1}, \ldots, g_{t}$ of an ideal $I$ is a Gröbner basis of I with respect to $\tau$ iff

$$
\left\langle L T_{\tau}\left(g_{1}\right), \ldots, L T_{\tau}\left(g_{t}\right)\right\rangle=\left\langle L T_{\tau}(I)\right\rangle
$$

where $\operatorname{LT}_{\tau}(I)=\left\{L T_{\tau}(f): f \in I\right\}$.

Theorem 4. Given a term ordering, every ideal I except $\{0\}$ has a Gröbner basis and any Gröbner basis is a basis of $I$.

Let us formally define the Quotient Basis $\mathrm{EST}_{\tau}$ that shall be used in the algorithm presented in section 4.2 .3 below.

Definition 15. [57] Quotient Basis

Let $A$ be a set of unique support points and $\tau$ a term ordering. A monomial basis of the set of polynomial functions over $A$ is

$$
E S T_{\tau}=\left\{x^{\alpha}: x^{\alpha} \notin\langle L T(g): g \in \operatorname{Ideal}(A)\rangle\right\}
$$

This definition is stating that $\operatorname{EST}_{\tau}$ comprises the elements $x^{\alpha}$ that are not divisible by any of the leading terms of the elements of the Gröbner basis of $\operatorname{Ideal}(A)$ (c.f. Definition 25 ii) in [57]).

Theorem 5. [57] The set EST $T_{\tau}$ has as many elements as there are support points.

For example, imagine that we have the $3 \times 8$ contingency table 4.1 and that we observe each support point with probability $q^{2}$.

Let us recall, the example from section 4.1.2, where we interpolated three points. Now the problem has increased a bit in complexity (from 3 to 8 points) and we want to compute the vanishing ideal (in this case and the example), the

\footnotetext{
${ }^{2}$ This is the table 6.8 obtained when we studied the dependence between preadmission use of statins and outcome shall be further studied in chapter 6 .
} 
Table 4.1: Contingency Table for Gröbner Basis

\begin{tabular}{|c|c|c|}
\hline$x$ & $y$ & $z$ \\
\hline 1 & 1 & 1 \\
\hline 2 & 1 & 1 \\
\hline 1 & 2 & 1 \\
\hline 2 & 2 & 1 \\
\hline 1 & 1 & 2 \\
\hline 2 & 1 & 2 \\
\hline 1 & 2 & 2 \\
\hline 2 & 2 & 2 \\
\hline
\end{tabular}

Algebraic Model is defined by zero-dimensional Variety (i.e. the set of uniquely observed points vanish in the Ideal). One way to calculate this vanishing ideal is by means of the Buchberger Algorithm [14]. However, for a given set of points, there is a more efficient algorithm based on specialized linear algebra techniques for zero-dimensional ideals using Indicator Polynomials (i.e. a polynomial that is 0 if $x \neq a$ and 1 if $x=1)$. This algorithm is called $M^{3}$ after its inventors (Marinari, Möller and Mora) [57],[59]. This method is implemented in the CoCoA package [60, 61].

We have calculated the Ideal of table 4.1 with the function IdealOfPoints [62] in $\boldsymbol{A} \boldsymbol{p} \boldsymbol{C o} \boldsymbol{C o} \boldsymbol{A}[61]$ and the lexicographic order. In our case the ideal is: $\left\langle z^{2}-3 z+2, y^{2}-3 y+2, x^{2}-3 x+2\right\rangle$, and its corresponding Gröbner basis is: $G=\left\{z^{2}-3 z+2, y^{2}-3 y+2, x^{2}-3 x+2\right\}$. It is interesting to see that this package constructs the Gröbner Basis equal to the Ideal (recall that every Gröbner Basis $G$ is also a basis of I) and also that the polynomials have as roots 1 and 2 (i.e. the coding values of our design matrix).

\subsubsection{Algorithm for Polynomial Regression/Interpolation of Observation Matrices}

Now we are ready to integrate all the definitions and theorems given so far in order to provide an algorithm for interpolation of designs or contingency tables and regression (recall the second example in section 4.1.2). First of all, let us summarize the following [63]:

- Let $A=\left(X_{1}, \cdots, X_{p}\right)$ be an $N \times p$ observation matrix of $N$ distinct support points in $\mathbb{Z}^{p 3}$. The $N$ distinct points can be represented as the set of solutions of the Gröbner Basis and a given term ordering $\tau$ (i.e. the evaluation of the observation matrix through the polynomials of the

\footnotetext{
${ }^{3}$ In section 4.3 we will further generalize the requirements for the distributions of these input sets.
} 
Grobner Basis):

$$
\left\{\begin{array}{c}
g_{1}(A)=0 \\
g_{2}(A)=0 \\
\vdots \\
g_{m}(A)=0
\end{array}\right.
$$

where $G=\left\{g_{1}, \cdots, g_{m}\right\}$ is the Gröbner Basis of $A$.

- By the Hilbert Basis Theorem 1, for a given term ordering $\tau$ and ideal $I$ any polynomial $p(x)$ can be written as

$$
p(x)=\sum_{j=1}^{m} I_{j}(A) g_{j}(A)+r(A)
$$

where $r(A)$ is unique.

- The monomials of $r(A)$ form a subset $\mathrm{EST}_{\tau}$, which comprises all monomials not divisible by the leading terms of $G$ for the given ordering $\tau$. Moreover, since $r(A)$ is unique, $\mathrm{EST}_{\tau}$ is also unique.

Now we are ready to give our algorithm for interpolation/regression ${ }^{4}$ :

1. Input: matrix with unique points $A$ and relative frequencies $q$. Without loss of generality this matrix could also be a transformed version of A by means of a Kernel.

2. Define a term ordering $\tau$ (for example lexicographic).

3. Calculate the ideal of matrix A (in our case, this is done with ApCoCoA) [61].

4. Calculate the reduced Gröbner Basis $G$ (this can be also calculated with the function IdealOfPoints [62] in ApCoCoA).

5. Identify the subset $\mathrm{EST}_{\tau}$ (i.e. identify the sub-set of monomials not divided by $G$ ).

6. Let $L$ be the logarithm of the monomials of $\operatorname{EST}_{\tau}$ (i.e. exponents). Write $\mathrm{EST}_{\tau}=\left\{a^{\alpha}\right\}_{\alpha \in L}$.

7. Write the polynomial interpolator as: $p(a)=\sum_{\alpha \in L} \eta_{\alpha} a^{\alpha}$.

8. Substitute the values of $a$ in $p\left(a_{k}\right)=q_{k} k \in\{1, \ldots, N\}$ and solve the polynomial system for the parameters $\eta_{\alpha}$. The solution is guaranteed and unique by the construction of $G$.

For example, a valid interpolation polynomial for table 4.1 is $f=-1 / 50 x y z+$ $3 / 100 x y+9 / 100 x z-3 / 25 y z-21 / 100 x+27 / 100 y+8 / 25 z+7 / 25$ and term ordering $x y z \succ x y \succ x z \succ x \succ y z \succ y \succ z \succ 1$. In this case, this interpolation is quite straightforward provided that the contingency table is fully observed. These polynomials become very useful for large contingency tables where we want to interpolate unobserved states (for example, in genomics or proteomics).

\footnotetext{
${ }^{4}$ The algorithm presented here goes beyond that presented in [63] in the sense that it is not only limited to Experimental Designs and also provides the interpolated values for the observed relative frequencies.
} 


\subsection{Regular Exponential Families}

Consider the sample space $\mathcal{X}$ with $\sigma$-algebra $\mathcal{A}$ on which a $\sigma$-finite measure $v$ is defined. Let $T: \mathcal{X} \rightarrow \mathbb{R}^{k}$ be a measurable map [64, 65]. Define the natural parameter space:

$$
N=\left\{\eta \in \mathbb{R}^{k}: \int_{\mathcal{X}} e^{\eta^{t} T(x)} d v(x)<\infty\right\}
$$

For $\eta \in N$, we can define a probability density $p_{\eta}$ on $\mathcal{X}$ as

$$
p_{\eta}(x)=e^{\eta^{t} T(x)-\phi(\eta)},
$$

where

$$
\phi(\eta)=\log \int_{\mathcal{X}} e^{\eta^{t} T(x)} d v(x)
$$

is the logarithm of the Laplace transform on $v^{t}$. Here $t$ denotes matrix/vector transpose. Let $P_{\theta}$ be the probability measure on $(\mathcal{X}, \mathcal{A})$ that has $v$-density $p_{\eta}$. Define $v^{t}=v \circ T^{-1}$ to be the measure that the statistic $T$ induces on the Borel $\sigma$-algebra of $\mathbb{R}^{k}$. The support of $v^{t}$ is the intersection of all closed sets $A \subseteq \mathbb{R}^{k}$ that satisfy $v^{t}\left(\mathbb{R}^{k} \backslash A\right)=0[58]$.

Definition 16. Let $k$ be a positive integer. The probability distributions $\left(P_{\eta} \mid \eta \in N\right)$ form a regular exponential family of order $k$ if $N$ is an open set in $\mathbb{R}^{k}$ and the affine dimension of the support $v^{t}$ is equal to $k$. The statistic $T(x)$ that induces the regular exponential family is called a canonical sufficient statistic.

Regular exponential families comprise the families of discrete distributions and Gaussian distributions that are subject to the work of this $\mathrm{PhD}$ thesis.

\subsubsection{Important Properties of Regular Exponential Fam- ilies}

Suppose $X$ is a random vector that is distributed according to some unknown distribution from a regular exponential family $\left(P_{\eta} \mid \eta \in N\right)$ of order $k$ with canonical sufficient statistic $T(x)$. Given an observation $X=x$, the log likelihood function takes the form:

$$
l(\eta \mid T(x))=\eta^{t} T(x)-\phi(\eta)
$$

where the log-Laplace function $\phi$ is a strictly convex and smooth function over the convex set $N$.

Theorem 6. [66]Convexity Property:

1. $N$ is a convex set and $\phi$ is convex on $N$.

2. $\phi$ is lower semi-continuous on $\mathbb{R}^{k}$ and is continuous on $N^{0}$.

3. $P_{\eta_{1}}=P_{\eta_{2}}$ iff the following convex combination is fulfilled:

$$
\phi\left(\alpha \eta_{1}+(1-\alpha) \eta_{2}\right)=\alpha \phi\left(\eta_{1}\right)+(1-\alpha) \phi\left(\eta_{2}\right)
$$

for some $\alpha \in(0,1)$. In this case 3 is valid for all $\alpha \in[0,1]$. 
4. It the order of the exponential family is $k$ (in particular, if $P_{\eta}$ is minimal), then $\psi$ is strictly convex on $N$, and $P_{\eta_{1}} \neq P_{\eta_{2}}$ for any $\eta_{1} \neq \eta_{2} \in N$.

Theorem 7. [66] Momentum Generation:

The derivatives of $\phi$ yield the moments of the canonical sufficient statistic such as the expectation and covariance matrix:

$$
\begin{gathered}
\zeta(\eta)=\frac{d}{d \eta} \phi(\eta)=E_{\eta}\{T(x)\} \\
\Sigma(\eta)=\frac{d^{2}}{d^{2} \eta} \phi(\eta)=E_{\eta}\left\{(T(x)-\zeta(\eta))(T(x)-\zeta(\eta))^{t}\right\}
\end{gathered}
$$

\subsubsection{Discrete Distributions as Regular Exponential Fam- ilies}

Let the sample space $\mathcal{X}$ be the set of integers $\{1, \ldots, m\}$. Let $v$ be the counting measure on $\mathcal{X}$ (the measure $v(A)$ of $A \subseteq X$ is equal to the cardinality of $A$ ). Consider the statistic $T \rightarrow \mathbb{R}^{m-1}$,

$$
T(x)=\left(I_{\{1\}(x)}, \ldots, I_{\{m-1\}(x)}\right)^{t},
$$

whose zero-one components indicate which value in $\mathcal{X}$ the argument $x$ is equal to. Also, when $x=m, T(x)=0$. The induced measure $v^{t}$ is a measure of the Borel $\sigma$-algebra of $\mathbb{R}^{m-1}$ with support equal to the $m$ vectors in $\{0,1\}^{m-1}$ that have at most one non-zero component. The differences of these vectors include all canonical basis vectors of $\mathbb{R}^{m-1}$. Thus the affine dimension of the support $v^{t}$ is equal to $m-1$.

It holds for all $\eta \in \mathbb{R}^{m-1}$ that

$$
\phi(\eta)=\log \left(1+\sum_{x=1}^{m-1} e^{\eta_{x}}\right)<\infty
$$

The natural parameter space $N$ is equal to all of $\mathbb{R}^{m-1}$ and in particular is open. The $v$-density $p_{\eta}$ is a probability vector in $\mathbb{R}^{m}$. The components $p_{\eta}(x)$ for $1 \leq x \leq m-1$ are positive and given by

$$
p_{\eta}(x)=\frac{e^{\eta_{x}}}{1+\sum_{x=1}^{m-1} e^{\eta_{x}}} .
$$

The last component of $p_{\eta}$ is also positive and equals

$$
p_{\eta}(m)=1-\sum_{x=1}^{m-1} p_{\eta}(x)=\frac{1}{1+\sum_{x=1}(m-1) e^{\eta_{x}}} .
$$

The family of the induced probability distribution $\left(P_{\eta} \mid \eta \in \mathbb{R}^{m-1}\right)$ is a regular exponential family of order $m-1$. The interpretation of the natural parameters $\eta_{x}$ is one of $\log$ odds because $p_{\eta}$ is equal to a given positive probability vector $\left(p_{1}, \ldots, p_{m}\right)$ if and only if $\eta_{x}=\log p_{x}-\log p_{m}$ for $x=1, \ldots, m-1$. This establishes a correspondence between the natural parameter space $N=\mathbb{R}^{m-1}$ and the interior of the $m-1$ dimensional probability simplex [58]. 


\subsubsection{Gaussian Distributions as Regular Exponential Fam- ilies}

Regarding Gaussian distributions, let the sample space $\mathcal{X}$ be the Euclidean space $\mathbb{R}^{p}$ equipped with its Borel $\sigma$-algebra and Lebesgue measure $v$. Let $T$ : $\mathcal{X} \rightarrow \mathbb{R}^{p} \times \mathbb{R}^{p(p+1) / 2}$ be given by:

$$
T(x)=\left(x_{1}, \ldots, x_{p},-x_{1}^{2} / 2, \ldots,-x_{p}^{2} / 2,-x_{1} x_{2}, \ldots,-x_{p-1} x_{p}\right)^{t} .
$$

The polynomial functions that form the components of $T(x)$ are linearly independent and, therefore, the support of $v^{t}$ has the full dimension $p+p(p+$ 1) $/ 2$.

If $\eta \in \mathbb{R}^{p} \times \mathbb{R}^{p(p+1) / 2}$, write $\eta_{[p]} \in \mathbb{R}^{p}$ for the vector of the first $p$ components $\eta_{i}, 1 \leq i \leq j \leq p$ and $\eta_{[p \times p]} \in \mathbb{R}^{p} \times p$ for the symetric matrix defined by the last $p(p+1) / 2$ components $\eta_{i j}, 1 \leq i \leq j \leq p$. The function $x \rightarrow e^{\eta^{t} T(x)}$ is $v$-integrable if and only if $\left.\eta_{[} p \times p\right]$ is positive definite. Therefore, the natural parameter space $N$ is equal to the Cartesian product $\mathbb{R}^{p}$ on the cone of positive definite $p \times p$ matrices. If $\eta$ is the open set $N$, then

$$
\phi(\eta)=-\frac{1}{2}\left(\log \operatorname{det}\left(\eta_{[p \times p]}\right)-\eta_{[p]}^{t} \eta_{[p \times p]} \eta_{[p]}-p \log (2 \pi)\right)
$$

Now the Lebesgue densities $p_{\eta}$ can be written as

$$
p_{\eta}(x)=\frac{1}{\sqrt{(2 \pi)^{p} \operatorname{det}\left(\eta_{[p \times p]}^{-1}\right)}} \exp \left(\eta_{[p]}^{t} x-\operatorname{Tr}\left(\eta_{[p \times p]} x x^{t}\right) / 2-\eta_{[p]}^{t} \eta_{[p \times p]} \eta_{[p]} / 2\right)
$$

Setting $\Sigma=\eta_{[p \times p]}^{-1}$ and $\mu=\eta_{[p \times p]}^{-1} \eta_{[p]}$, we find that

$$
p_{\eta}(x)=\frac{1}{\sqrt{(2 \pi)^{p} \operatorname{det}(\Sigma)}} \exp \left(-\frac{1}{2}(x-\mu)^{t} \Sigma^{-1}(x-\mu)\right)
$$

is the density of the multivariate normal distribution $\mathcal{N}_{p}(\mu, \Sigma)$. Therefore, the family of all multivariate normal distributions on $\mathbb{R}^{p}$ with positive definite covariance matrix is a regular exponential family of order $p+p(p+1) / 2$ [58].

\subsection{Algebraic Exponential Families}

We know from definition 10 that a model that can be expressed by means of a variety is a algebraic model. Taking this definition one step further (definition 11), a Statistical Model that can be expressed as a Variety is defined as an Algebraic Statistical Model.

More formally, a statistical model [67] is a set of probability distributions on the sample space $\mathcal{S}$. A parameterized statistical model is a parameter set $\Theta$ together with a function $P: \Theta \rightarrow P(\mathcal{S})$, which assigns to each parameter point $\theta \in \Theta$ a probability distribution $P_{\theta}$ on $\mathcal{S}$. Of course, by the definition above, Exponential Families are naturally defined as Statistical Models. Moreover, they can be expressed by means of reparameterization as Algebraic Statistical Models (definition 11). Therefore, the statistical properties of exponential families 
are determined by the geometry of their parameter spaces [58, 66]. This suggests that if the parameter spaces have an algebraic structure then the tools of computational algebraic geometry can be employed to address questions arising in inference theory and Machine Learning. Semi-algebraic sets, as employed in the following definition [58], provide the necessary flexibility to capture the algebraic structure found in the models developed in this $\mathrm{PhD}$ thesis.

\subsubsection{Semi-Algebraic sets}

Loosely speaking a semi-algebraic set is simply a set that can be described with a finite number of polynomial equalities and inequalities. A variety is clearly a semi-algebraic set and also the interpolation polynomials and Gröbner bases described above.

Definition 17. [58] A basic semi-algebraic set is a is subset of points in $\mathbb{R}^{n}$ of the form

$$
A_{F, H}=\left\{\theta \in \mathbb{R}^{n} \mid \quad f(\theta)>0 \quad \forall f \in F, \quad h(\theta)=0 \quad \forall h \in H\right\}
$$

where $F \subset \mathbb{R}[\mathbf{t}]$ is a finite (possibly empty) collection of polynomials and $H \subseteq$ $\mathbb{R}[\mathbf{t}]$ in an arbitrary (possibly empty) collection of polynomials. A semi-algebraic set is a finite union of basic semi-algebraic sets. If $F=\emptyset$ then $A$ is called a real algebraic variety (see definition 9).

A general semi-algebraic set occurs when we consider sets of the form

$$
A_{F, G, H}=\left\{\theta \in \mathbb{R}^{n} \mid \quad f(\theta)>0 \quad \forall f \in F, \quad g(\theta) \geq 0, \quad \forall g \in G, \quad h(\theta)=0, \quad \forall h \in H\right\}
$$

where both $F$ and $G$ are finite collections of real polynomials.

An example of a semi-algebraic set is the set of $m \times m$ positive definite matrices $\Sigma$, where $F$ consists of all principal sub-determinants of a symmetric matrix $\Psi$ and $G, H$ are the empty set.

Definition 18. [58/ Algebraic Exponential Family

Let $\left(P_{\eta} \mid \eta \in N\right)$ be a regular exponential family of order $k$. The subfamily induced by the set $M \subseteq N$ is an algebraic exponential family if there exists an open set $\bar{N} \subseteq \mathbb{R}^{k}$, a diffeomorfism $g: N \rightarrow \bar{N}$, and a semi-algebraic set $A \subseteq \mathbb{R}^{k}$ such that $M=g^{-1}(A \cap \bar{N})$.

The definition states that an algebraic exponential family is given by a semialgebraic subset of the parameter space of a regular exponential family. This parameter space may be obtained by a re-parametrization $g$ of the natural parameter space $N$.

Definition 19. [58, 68] Rational Mappings

Let $\psi_{1}=\frac{f_{1}}{g_{1}}, \ldots, \psi_{n}=\frac{f_{n}}{g_{n}}$ be rational functions where $f_{i}, g_{i} \in \mathbb{R}[\mathbf{x}]=$ $\mathbb{R}\left[x_{1}, \ldots, x_{d}\right]$ are real polynomial functions. Then a rational map is defined by:

$$
\psi: \mathbb{R}^{d} \rightarrow, \quad \mathbf{a} \mapsto\left(\psi _ { 1 } \left((a), \ldots, \psi_{n}((a))\right.\right.
$$

The rational map is a well-defined function on the open set $D_{\psi}=\left\{\mathbf{a} \subset \mathbb{R}^{d}\right.$ : $\left.\prod g_{i}(\mathbf{a}) \neq 0\right\}$. 
Theorem 8. [58] Tarski-Seidenberg

Let $A_{F, H} \subseteq \mathbb{R}^{d}$ be a semi-algebraic set and $\psi$ a rational map that is well defined on $A_{F, H}$, that is, $A_{F, H} \subseteq D_{\psi}$. Then the image $\psi\left(A_{F, H}\right)$ is also semialgebraic set.

Definition 20. The open probability simplex is defined as

$$
\Delta_{k-1}=\left\{\left(p_{1}, \ldots, p_{k}\right) \in \mathbb{R}^{k}: p_{1}, \ldots, p_{k}>0 \text { and } \sum_{i=1}^{k} p_{i}=1\right\}
$$

Remark 1. [68] The open probability simplex for discrete random variables is a basic semi-algebraic set, where $F=\left\{x_{i} \mid i=1, \ldots, n-1\right\} \bigcup\left\{1-\sum_{i=1}^{n-1} x_{i}\right\}$ and $H=\emptyset$. From a topological point of view, the relative interior of any convex polyhedron in any dimension is a basic semi-algebraic set, while the whole polyhedron is a basic semi-algebraic set.

Remark 2. [68] The set $\Sigma \subset \mathbb{R}^{m \times m}$ of positive definite matrices is a basic semialgebraic set, where $F$ consists of all principal sub-determinants of a symmetric matrix $\Psi$, and $G$ is the empty set.

\subsubsection{Independence Models and Algebraic Exponential Fam- ilies}

The statistical models defined in this $\mathrm{PhD}$ thesis are based on conditional independence considerations. In our case, we will study models for testing independence hypothesis in contingency tables, which can be related to graphical models by means of the Hammersley-Clifford Theorem such as Markov Chains or Lattices. In this section, we show that conditional independence yields algebraic exponential families for both the Gaussian and Discrete cases.

Ideals (see definition 12) can be used to determine real algebraic varieties by computing the zero set of the ideal:

$$
V(I)=\left\{a \in \mathbb{R}^{n} \mid f(a)=0, \forall f \in I\right\} .
$$

Reversing this procedure, if we are given a set $V \subset \mathbb{R}^{n}$ we can compute its defining ideal, which is the set of polynomials that vanish on $V$ :

$$
I(V)=\{f \in \mathbb{R}[x] \mid f(a)=0, \forall a \in V\} .
$$

Definition 21. [58] Invariant

Let $A$ be a semi-algebraic set defining an algebraic exponential family $\mathcal{P}_{M}=$ $\left\{P_{\eta} \mid \eta \in M\right\}$ via $M=g^{-1}(A \cap g(n))$. A polynomial in the ideal $I(A)$ is a model invariant for $\mathcal{P}_{M}$. below.

Conditional independence can be studied by means of the definitions given

Definition 22. $|58| A$ set of indeterminates $x_{i_{1}}, \ldots, x_{i_{k}}$ is algebraically independent for the ideal $I$ if there is no polynomial in $p_{i_{1}}, \ldots, p_{i_{k}}$ that belongs to I

Proposition 1. [58] The dimension of $A$ is the cardinality of the largest set of algebraically independent indeterminates for $I(A)$. 


\section{Conditional Independence for Gaussian Distributions}

Let $X=\left(X_{1}, \ldots, X_{p}\right)$ be a random vector with joint normal distribution $\mathcal{N}_{p}(\mu, \Sigma)$ with mean vector $\mu \in \mathbb{R}^{p}$ and positive definite covariance matrix $\Sigma$. For three pairwise disjoint index sets $A, B, C \subseteq\{1, \ldots, p\}$, the sub-vectors $X_{A}$ and $X_{B}$ are conditionally independent given $X_{C}$, in symbols $X_{A} \Perp X_{B} \mid X_{C}$, iff

$$
\operatorname{det}\left(\Sigma_{A \cup C \times B \cup C}\right)=0 .
$$

Here $A \cup C \times B \cup C$ is the minor related to the variables over which we are calculating the marginal dependence/independence (i.e. the resulting minor after removing the rows and columns corresponding to the conditional independence statement).

If $C=\emptyset$, then conditional independence given $X_{\emptyset}$ is understood to mean marginal independence $X_{A}$ and $X_{B}$. Here, equation 4.38 gives the semi-algebraic set that allows to see the conditional independence for a Gaussian distribution as an algebraic exponential family [58, 68].

For example, let $X=\left(X_{1}, X_{2}, X_{3}\right)$ have a trivariate normal distribution $\mathcal{N}_{3}(\mu, \Sigma)$ and define a model requiring $X_{1} \Perp X_{2} \mid X_{3}$. This model is an algebraic exponential family given by the subset $M=\zeta^{-1}(V \cap \zeta(N))$, where $\zeta(N)$ is the Gaussian mean parameter space and the algebraic variety is:

$$
A=\left\{(\mu, \Sigma) \in \mathbb{R}^{3} \times \mathbb{R}_{\mathrm{sym}}^{3 \times 3} \mid \operatorname{det}\left(\sigma_{\{1,3\} \times\{2,3\}}\right)=\sigma_{12} \sigma_{3,3}-\sigma_{13} \sigma_{23}=0\right\}
$$

\section{Conditional Independence for Discrete Data}

Let a set of discrete random variables $X_{1}, \ldots, X_{n}$ where $X_{i}$ takes values over the probability space $\Xi_{i}$. Then a distribution over the sample space $\Xi_{1} \times \cdots \times \Xi_{n}$ is equivalent to a matrix $\left(p_{i_{1}, \ldots, i_{n}}\right) \in \Xi_{1} \times \cdots \times \Xi_{n}$ where $p_{i_{1}, \ldots, i_{n}}=\operatorname{Prob}\left(X_{1}=\right.$ $\left.i_{1}, \ldots, X_{n}=i_{n}\right)$.

Definition 23. Given three disjoint subsets $A, B, C \neq \varnothing$ of $\left\{X_{1}, \ldots, X_{n}\right\}, A$ is independent of $B$ given $C, A \Perp B \mid C$ if $\operatorname{Prob}(A=a, B=b \mid C)=\operatorname{Prob}(A=$ $a \mid C=c) \operatorname{Prob}(B=b \mid C=c) \forall a, b, c$ such that $\operatorname{Prob}(C=c)>0$.

Proposition 2. A probability distribution $P=\left(p_{i_{1}, \ldots, i_{n}}\right)$ satisfies $A \Perp B \mid C$ iff

$$
\begin{gathered}
P_{a, b, c} P_{a^{\prime}, b^{\prime}, c}=P_{a, b^{\prime}, c} P_{a^{\prime}, b, c} . \\
\forall a, a^{\prime} \in \prod_{x_{i} \in A} \Xi_{i}, \\
\forall b, b^{\prime} \in \prod_{x_{j} \in B} \Xi_{j}, \\
\forall c \in \prod_{x_{k} \in C} \Xi_{k},
\end{gathered}
$$

where

$$
\begin{gathered}
P_{a, b, c}=\operatorname{Prob}(A=a, B=b, C=c) \\
P_{a^{\prime}, b^{\prime}, c}=\operatorname{Prob}\left(A=a^{\prime}, B=b^{\prime}, C=c\right) \\
P_{a, b^{\prime}, c}=\operatorname{Prob}\left(A=a, B=b^{\prime}, C=c\right) \\
P_{a^{\prime}, b, c}=\operatorname{Prob}\left(A=a^{\prime}, B=b, C=c\right) .
\end{gathered}
$$

Proof. We want to show Eq.(4.40), so we rewrite:

$$
\begin{array}{r}
P(a, b \mid c) P(c) P\left(a^{\prime}, b^{\prime} \mid c\right) P(c)= \\
P\left(a, b^{\prime} \mid c\right) P(c) P\left(a^{\prime}, b \mid c\right) P(c) .
\end{array}
$$


Taking into account definition 23, the left hand side of this equation becomes

$$
P(a, b \mid c) P\left(a^{\prime}, b^{\prime} \mid c\right)=P(a \mid c) P(b \mid c) P\left(a^{\prime} \mid c\right) P\left(b^{\prime} \mid c\right)
$$

whereas, also by definition 23, its right hand side becomes

$$
P\left(a, b^{\prime} \mid c\right) P\left(a^{\prime}, b \mid c\right)=P(a \mid c) P\left(b^{\prime} \mid c\right) P\left(a^{\prime} \mid c\right) P(b \mid c),
$$

and, thus, the proposition holds. We are only left now to show that proposition 2 implies definition 23. For this, note that

$$
\begin{array}{r}
P(A=a \mid C=c) P(B=b \mid C=c)= \\
\sum_{b^{\prime}, a^{\prime}} P\left(a, b^{\prime} \mid c\right) P\left(a^{\prime}, b \mid c\right)= \\
\sum_{b^{\prime}} P\left(a, b^{\prime} \mid c\right) \sum_{a^{\prime}} P\left(a^{\prime}, b \mid c\right) \\
\sum_{b^{\prime}, a^{\prime}} P(a, b \mid c) P\left(a^{\prime}, b^{\prime} \mid c\right)= \\
P(a, b \mid c) \sum_{a^{\prime}, b^{\prime}} P\left(a^{\prime}, b^{\prime} \mid c\right)=P(a, b \mid c) .
\end{array}
$$

Definition 24. The conditional independence ideal $I_{A \Perp B \mid C}$ is generated by all quadratic polynomials in proposition 2

Equivalently, this definition implies that the rank of $M_{c}$ is $\leq 1$ where:

$$
M_{c}=\left(\begin{array}{cc}
P_{a, b, c} & P_{a, b^{\prime}, c} \\
P_{a^{\prime}, b, c} & P_{a^{\prime}, b^{\prime}, c}
\end{array}\right)_{\forall c \in \prod_{x_{k} \in C} \Xi_{k}},
$$

which, as in the Gaussian case, gives the semi-algebraic set that allows us to check marginal independence for the algebraic exponential family.

\subsubsection{Factorization of Discrete Distributions and Graphi- cal Models}

A very important consequence of proposition 2 for multinomial distributions is that conditional independence models can be compactly modelled by graphical models via the Hammersley Clifford theorem, which also lend significant savings to the computational tasks via factorization of the joint distribution. In this section we introduce the definition of Undirected Graphical Models or Markov Random Fields [69].

\subsubsection{Markov Random Fields and Graphical Models}

Definition 25. [69] Graph Separation

Given an undirected graph $\mathcal{G}=(V, E)$ where $V$ and $E$ are the set of nodes and edges respectively, let $A, B, C$ be disjoint subsets of nodes. If every path from $A$ to $B$ includes at least one node from $C$, then $C$ is said to separate $A$ from $B$ in $G$. 
Definition 26. [69] Markov Random Field

Given and undirected graph $\mathcal{G}$, a Markov Random Field (MRF) is defined as a set of probability distributions $M R F_{\mathcal{G}}:=\{p(\mathbf{x}): p(\mathbf{x})>0, \forall p, \mathbf{x}\}$ such that $\forall p \in M R F_{\mathcal{G}}$ and for any three disjoint subsets $A, B, C$ of $\mathcal{G}$, if $C$ separates $A$ from $B$ then $p$ satisfies $X_{A} \Perp X_{B} \mid X_{C}$. If $p \in M R F_{\mathcal{G}}$, we often say $p$ respects $\mathcal{G}$.

With these two definitions [70] it is relevant to ask the following questions:

- Given a graph $\mathcal{G}$ and $p \in M R F_{\mathcal{G}}$, how can we efficiently check all the conditional independence relationships encoded in it? This is normally done by means of the independence definitions above that translate into the Markov condition for Graphical Models (i.e. each node is independent of its non-descendants).

- Given a set of conditional independence relationships, how can we obtain a valid $\mathcal{G}$ ? This is also done by means of studying the marginal independences presented above.

- For all distributions in $M R F_{\mathcal{G}}$, how should their $p d f$ look like? The Hammersley Clifford theorem shows that these $p d f$ should factorize as we will show below.

Definition 27. [71] Cliques and Maximal Cliques

A clique of a graph is a sub-graph of it where each pair of nodes is connected by an edge. The maximal clique of a graph is a clique which is not a proper subset of another clique.

The set of maximal cliques is normally denoted by $\mathcal{C}$.

Definition 28. [69, 71] Factorization

A pdf $p(\mathbf{x})$ is said to factorize wrt a given undirected graph $\mathcal{G}$ it can be written as:

$$
p(\mathbf{x})=\frac{1}{Z} \prod_{c \in \mathcal{C}} \psi_{c}\left(x_{c}\right),
$$

where $\psi_{c}$ is a general non-negative real valued function called the potential function. The constant $Z$ ensures $\int p(\mathbf{x}) d \mathbf{x}=1$.

This definition together with proposition 2 provides a rigorous form for a $p d f$ based on the maximal cliques. The following two theorems close the loop between conditional independence statements and the graph $\mathcal{G}$.

Theorem 9. If a pdf $p$ factorizes according to an undirected graph $\mathcal{G}$, then $p \in$ $M R F_{\mathcal{G}}$, i.e., if $A, B$ and $C$ are disjoint subsets of nodes such that $C$ separates $A$ from $B$ in $G$, then $p$ satisfies $X_{A} \Perp X_{B} \mid X_{C}$.

Proof. The proof is completed by applying definition 26 to proposition 2 .

\section{Theorem 10. Hammersley Clifford}

If a pdf $p \in M R F_{\mathcal{G}}$, then $p(\mathbf{x})$ must also factorize according to $\mathcal{G}$, i.e. there exist functions $\psi_{c}(\mathbf{x})$ on $c \in \mathcal{C}$, such that

$$
p(\mathbf{x})=\frac{1}{Z} \exp \left(\sum_{c \in \mathcal{C}} \psi_{c}\left(x_{c}\right)\right) .
$$


Remark 3. For the particular case of regular exponential families, theorem 9 shows that if the sufficient statistics $T$ and natural parameters $\eta$ of a regular exponential family factorize onto the cliques of a Graph $\mathcal{G}$ by $\left\{T_{c}\right\}_{c \in C}$ and $\{\eta\}_{c \in \mathcal{C}}$ respectively:

$$
p(x, \eta)=\exp \left(\sum_{c \in \mathcal{C}} \eta_{c}^{t} T\left(x_{c}\right)-\phi(\eta)\right),
$$

then all the distributions in $P_{T}(\mathcal{S})$ (i.e. all the distributions in the statistical model) must respect $\mathcal{G}$.

Theorem 11. [69]

If all distributions in $P_{T}(\mathcal{S})$ respect $\mathcal{G}$, then $T$ and $\eta$ must factorize onto the cliques by $\left\{T_{c}\right\}_{c \in \mathcal{C}}$ and $\{\eta\}_{c \in \mathcal{C}}$ respectively.

It is interesting to use the data distribution to find a valid Graph $\mathcal{G}$ since it allows us to study the different relations between the input variables of our model. If we restrict to a regular exponential family with specificied sufficient statistics $T$ that factorize according to $\mathcal{G}$, then the distribution is guaranteed to respect $\mathcal{G}$ and we only need to estimate the clique-wise natural parameters. This gives a parametric model since $T_{c}(x)$ are fixed.

Definition 29. [71] (Bayes Networks from MRF)

$\mathcal{K}$ is a Bayesian network with respect to graph $\mathcal{G}$ if its joint probability density function factorizes as a product of the individual density functions, conditional on their parent variables:

$$
p(x)=\prod_{v \in V} p\left(X_{v} \mid X_{p a(v)}\right) .
$$

where $p a(v)$ is the set of neighbours of $v$ and $V$ is the set of marginally dependent variables.

Remark 4. From this definition and theorem 10 we know that if $\mathcal{G}$ is a $D A G$, then the pairwise Markov Condition (i.e. each node is independent of its non descendants) will hold. In other words, our support $\left\{X_{1}, \ldots, X_{n}\right\}$ will define a Markov Field.

Theorem 12. [69, 72] Recursive Factorization

A probability density $p$ satisfies the factorization property with respect to the directed acyclic graph $\mathcal{G}$ iff it satisfies the local Markov property.

Remark 5. The local Markov property associated with the directed acyclic graph $\mathcal{G}$ is the set of conditional independence statements (CI):

$$
\operatorname{local}(\mathcal{G})=\{u \Perp(n d(u) \backslash p a(u)) \mid p a(u): u=1, \ldots, n\} .
$$

Here $n d$ stands for non-descendant node. 


\subsection{Kernels: Definitions and Properties}

For the sake of generality, we consider in this section all functions to be complex valued, unless otherwise stated. So, in what follows, if $z$ is a complex number, we denote its conjugate by $\bar{z}$. Also, $z \geq 0$ means $\operatorname{Re}(z) \geq 0$ and $\operatorname{Im}(z)=0$. If $X$ is a matrix, $X^{*}$ denotes its conjugate transpose. A positive semi-definite matrix $K$ is a hermitian matrix whose eigenvalues are real and non-negative. A squared matrix may be seen as a function defined on $I \times I$, where $I$ is the finite set of indices. The following is a generalization of this concept to functions whose domain $X \times X$ is not necessarily finite. Here, $X$ is a non-empty set. The reader will find further details about the background on Topology and Measure theory used throughout this section.

Definition 30. [73/ Kernel Function

$A$ kernel is a function $k$ that for all $\mathbf{x}, \mathbf{z} \in X$ satisfies

$$
k(\mathbf{x}, \mathbf{z})=\phi(\mathbf{x}) \cdot \phi(\mathbf{z}),
$$

where $\phi$ is a mapping from $X$ to a measurable feature space $F$ and $\cdot$ is the inner product (see definition 59) in $F$

$$
\Phi: x \mapsto \phi(\mathbf{x}) \in F .
$$

Definition 31. [73] Gram Matrix/Kernel Matrix

Given the set of vectors $\left\{x_{1}, \ldots, x_{n}\right\}$, the Gram Matrix is defined as the $n \times n$ matrix $K$ whose entries are $K_{i j}=x_{i} \cdot x_{j}$. If we are using a kernel function $k$ to evaluate the inner products in a feature space with feature map $\phi$, the associated kernel matrix has entries

$$
K_{i j}=\phi\left(x_{i}\right) \cdot \phi\left(x_{j}\right)=k\left(x_{i}, x_{j}\right) .
$$

Definition 32. [74, 75] Positive Definite Kernel

A kernel $\varphi: X \times X \rightarrow \mathbb{C}$ is called a positive semi-definite iff it is hermitian $(\varphi(y, x)=\overline{\varphi(x, y)} \forall x, y \in X)$ and

$$
\sum_{i=1}^{n} \sum_{j=1}^{n} c_{i} \overline{c_{j}} \varphi\left(x_{i}, x_{j}\right) \geq 0
$$

$\forall n \in \mathbb{N},\left\{x_{1}, \ldots, x_{n}\right\} \subseteq X$ and $\left\{c_{1}, \ldots, c_{n}\right\} \subseteq \mathbb{C}$. If for any distinct $x_{1}, \ldots, x_{n}$, the equality in (4.51) implies $c_{1}=\cdots=c_{n}=0$, then the kernel $\varphi$ is called strictly positive kernel.

Definition 33. [74, 75] Negative Definite Kernel

A kernel $\psi: X \times X \rightarrow \mathbb{C}$ is called a conditionally negative definite iff:

- $\psi$ is hermitian (i.e. $\psi(y, x)=\overline{\psi(x, y)} \forall x, y \in X)$.

- $\forall n \in \mathbb{N},\left\{x_{1}, \ldots, x_{n}\right\} \subseteq X$ and $\left\{c_{1}, \ldots, c_{n}\right\} \subseteq \mathbb{C}$ with $\sum_{i=1}^{n} c_{i}=0$ it holds

$$
\sum_{i=1}^{n} \sum_{j=1}^{n} c_{i} \overline{c_{j}} \psi\left(x_{i}, x_{j}\right) \leq 0 .
$$

If for any distinct $x_{1}, \ldots, x_{n}$, the equality in equation 4.52 implies $c_{1}=\cdots=$ $c_{n}=0$, then the kernel $\psi$ is called strictly negative definite kernel. If $\psi$ is strictly negative definite, we call $-\psi$ strictly conditionally positive definite [74][75]. 


\subsubsection{Important Properties of Positive and Negative Def- inite Kernels}

Property 1. [75] If $\varphi$ is positive definite, then $\forall x, y \in X$ :

$$
|\varphi(x, y)|^{2} \leq \varphi(x, x) \varphi(y, y)
$$

Property 2. [75] If $\psi$ is negative definite, then $\forall x, y \in X$ :

$$
\psi(x, x)+\psi(y, y) \leq 2 \operatorname{Re}(\psi(x, y)) .
$$

Property 3. [75] Separability Any $\varphi$ of the form $\varphi(x, y)=f(x) \overline{f(y)}$, where $f: X \rightarrow \mathbb{C}$ is an arbitrary function, is positive definite. In particular, a constant kernel $(x, y) \mapsto c$ is positive definite iff $c \geq 0$.

Let $\mathcal{K}_{+}$and $\mathcal{K}_{-}$respectively denote the sets of positive and negative definite kernels. Their strict counterparts are accordingly denoted as $\mathcal{K}_{++}$and $\mathcal{K}_{--}$.

Definition 34. A convex cone is a subset of a vector space over an ordered field that is closed under linear combinations with positive coefficients.

Property 4. $\mathcal{K}_{+}$and $\mathcal{K}_{-}$are both convex cones, closed in the topology of point wise convergence.

This property means that if $\varphi_{1}$ and $\varphi_{2}$ are positive (resp. negative) definite, so is $\lambda_{1} \varphi_{1}+\lambda_{2} \varphi_{2}$ for any non-negative scalars $\lambda_{1}, \lambda_{2}$, and that if $\left(\varphi_{n}\right)_{n \in \mathbb{N}}$ is a sequence of positive (resp. negative) definite kernels converging point wise to $\varphi$, then $\varphi$ is positive (resp. negative) definite. Regarding integrals as limits of weighted sums, it also implies that $\mathcal{K}_{+}$and $\mathcal{K}_{-}$are closed under point wise integration.

Property 5. If $\left(\varphi_{\theta}\right)_{\theta \in \Theta}$ is a family of positive (resp. negative) definite kernels and $\mu$ is a positive measure on $\Theta$ such that $\varphi_{\theta}(x, y)$ is $\mu$ integrable $\forall x, y \in X$, then $\varphi: X \times X \rightarrow \mathbb{C}$ defined by

$$
\varphi(x, y)=\int_{\Theta} \varphi_{\theta}(x, y) d \mu(\theta)
$$

is positive (resp. negative) definite.

This property (5) along with the following will enable us to define kernels from the Algebraic Statistical Models for the re-parametrized Regular Exponential Families.

Property 6. Closure under products

If $\varphi_{1}$ and $\varphi_{2}$ are positive definite, so is $\varphi_{1} \varphi_{2}$.

Property 7. [74, 75] polynomial combination

Let $\varphi$ be a positive definite kernel. Any polynomial combination with nonnegative coefficients, $\sum_{i=0}^{n} \lambda_{i} \varphi^{i}$ with each $\lambda_{i} \geq 0$, is positive definite. Furthermore, if $|\varphi(x, y)|<\rho \leq \infty$ and $f: \mathbb{C} \rightarrow \mathbb{C}$ is a holomorphic function in $\{z \in \mathbb{C}:|z|<\rho\}, f(z)=\sum_{n=0}^{\infty} a_{n} z^{n}$, where each $a_{n} \geq 0$, then $f \circ \varphi$ is positive definite. In particular, $e^{\varphi}$ is positive definite. 


\subsubsection{Relation between Positive and Negative Definite Ker- nels}

Property 8. [76, 77, 78] Centering

Let $\psi: X \times X \rightarrow \mathbb{C}$ be an hermitian function and $x_{0} \in X$. Define $\varphi_{0}, \varphi$ : $X \times X \rightarrow \mathbb{C}$ by:

$$
\varphi_{0}(x, y)=\psi\left(x, x_{0}\right)+\psi\left(y, x_{0}\right)-\psi(x, y)
$$

and

$$
\varphi(x, y)=\varphi_{0}(x, y)-\psi\left(x_{0}, x_{0}\right)
$$

Then:

- $\varphi_{0}$ is positive definite iff $\psi$ is negative definite,

- If $\psi\left(x_{0}, x_{0}\right) \geq 0$, then $\varphi$ is positive definite iff $\psi$ is negative definite.

Property 9. [76, 77, 78] Exponentiation

The kernel $\psi: X \times X \rightarrow \mathbb{C}$ is negative definite iff $e^{-t \psi}$ is positive definite $\forall t>0$.

Property 10. [76, 77, 78] Inversion $\forall t>0$.

The kernel $\psi: X \times X \rightarrow \mathbb{C}^{+}$is negative definite iff $\frac{1}{t+\psi}$ is positive definite

\section{Hilbert Representation of Kernels}

The following properties show that positive or negative definite kernels can be represented as an inner product or squared distance induced from the inner product in a Hilbert space $H$ by means of a feature mapping $\Psi: X \rightarrow H$ that maps each data point $x \in X$ to its feature representation $\Psi(x)$. The idea here (kernel trick) is never to perform direct computations in $H$, which has often very high dimension (even infinite), but instead use the kernel function in $X$ to compute inner products or distances in $H$. The following property is the obvious particular case of definition 30 to Hilbert spaces.

Property 11. [77] A function $\varphi: X \times X$ is a positive definite (PSD) kernel iff there is a Hilbert space $H$ and a mapping $\Phi$

$$
\Phi: X \mapsto H
$$

such that

$$
\varphi(x, y)=\Phi(x) \cdot \Phi(y)
$$

for all $x, y \in X$.

Property 12. [77] A function $\Psi: X \times X$ is a negative definite kernel iff there is a Hilbert space $H$, a mapping $\Phi: X \rightarrow H$ and a function $f: X \rightarrow \mathbb{C}$ such that

$$
\psi(x, y)=\|\Phi(x)\|^{2}+\|\Phi(y)\|^{2}-2 \Phi(x) \cdot \Phi(y)+f(x)+\overline{f(x)}
$$

for all $x, y \in X$. Moreover, 
- If there is some $x_{0} \in X$ such that $\psi\left(x, x_{0}\right) \in \mathbb{R}$ for all $x \in X$, and if $\psi$ vanishes on the diagonal $\psi(x, x)=0$, then one can choose $f=0$.

- If $\psi$ is real-valued, $H$ may be chosen as a real Hilbert space and equation (12) becomes

$$
\psi(x, y)=\|\Phi(x)-\Phi(y)\|^{2}+f(x)+\overline{f(y)} .
$$

- If $\psi$ is real-valued and vanishes on the diagonal then in addition $f=0$, so $\psi$ admits the representation:

$$
\psi(x, y)=\|\Phi(x)-\Phi(y)\|^{2} .
$$

This means that $\sqrt{\psi}$ is a semi metric on $X$ such that $\Psi$ is an isometry. Furthermore, if $\phi(x, y)=0$ iff $x=y$, then $\sqrt{\psi}$ is a metric.

\subsubsection{Reproducing Kernel Hilbert Spaces}

Associated with a PSD kernel $k$ is a reproducing kernel Hilbert space $H$. It is a set of functions which is constructed in the following steps. First include the span of $k(x, \cdot)$ for all $x \in X$ :

$$
H_{\frac{1}{2}}=\left\{\sum_{i=1}^{n} a_{i} k\left(x_{i}, \cdot\right): n<\infty, a_{i} \in \mathbb{C}, x_{i} \in X\right\} .
$$

Second, define an inner product between $f=\sum_{i=1}^{n} \alpha_{i} k\left(x_{i}, \cdot\right)$ and $g=\sum_{j=1}^{m} \beta_{j} k\left(x_{j}^{\prime}, \cdot\right)$ :

$$
f \cdot g=\sum_{i=1}^{n} \sum_{j=1}^{m} \alpha_{i} \beta_{j} k\left(x_{i}, x_{j}^{\prime}\right)=\sum_{j=1}^{m} f\left(x_{j}^{\prime}\right)=\sum_{i=1}^{n} \alpha_{i} g\left(x_{i}\right) .
$$

Although the definition depends on the specific expansion of $f$ and $g$ which may not be unique, it is still well defined because the last two equalities show that the value is independent of the coefficients $\alpha_{i}, x_{i}, \beta_{j}, x_{j}^{\prime}$ given $f$ and $g$. The other properties required by the inner product are clearly satisfied (bilinear, hermitian and positive-definite $(f \cdot f \geq 0)$ ). Since $f \cdot k(x, \cdot)=f(x)$ for all $f, k$ is called reproducing kernel.

This inner product and its induced metric further allow us to complete the space $H_{\frac{1}{2}}$. We define the completed space as the RKHS induced by $k$ :

$$
H=\overline{H_{\frac{1}{2}}}=\overline{\operatorname{span}\left\{k\left(x_{i}, \cdot\right): x_{i} \in X\right\}} .
$$

The inner product defined on $H_{\frac{1}{2}}$ is extended to $H$ so $H$ is a Hilbert space.

\subsubsection{Kernels as Covariance Functions}

Theorem 13. Mercer

Let $X$ be a compact subset of $\mathbb{R}^{n}$ (cf. appendix $A$ ). Suppose $k$ is a continuous symmetric function such that the integral operator $T_{k}: L_{2}(X) \rightarrow L_{2}(X)$

$$
\left(T_{k}(f)\right)(\cdot)=\int_{X} k(\cdot, x) f(x) d x,
$$


is positive, that is

$$
\int_{X \times X} k(x, z) f(x) f(z) d x d z \geq 0,
$$

for all $f \in L_{2}(X)$. Then we can expand $k(x, z)$ in a uniformly convergent series on $X \times X$ in terms of functions $\phi_{j}$, satisfying $\phi_{j} \cdot \phi_{i}=\delta_{i j}$

$$
k(x, z)=\sum_{j=1}^{\infty} \phi_{j}(x) \phi_{j}(z) .
$$

Furthermore, the series $\sum_{i=1}^{\infty}\left\|\phi_{i}\right\|^{2}$ is convergent.

Theorem 13 enables us to express a kernel as a sum over a set of functions of the product of their values on the two inputs [79]

$$
k(x, z)=\sum_{i=1}^{\infty} \phi_{i}(x) \phi_{j}(z) .
$$

This suggests a different view of kernels as a covariance function determined by a probability distribution over a function class. In general, given a distribution $q(f)$ over a function class $\mathcal{F}$, the covariance function is given by

$$
k_{q}(x, z)=\int_{\mathcal{F}} f(x) f(z) q(f) d f .
$$

Also following [79], we will show that every kernel can be obtained as a covariance kernel in which the distribution has a particular form. Given a valid kernel $k$, consider the Gaussian prior $q$ that generates functions $f$ according to

$$
f(x)=\sum_{i=1}^{\infty} u_{i} \phi_{i}(x),
$$

where $\phi_{i}$ are the orthonormal functions of theorem 13 for the kernel $k$, and $u_{i}$ are iid according to the Gaussian distribution $N(0,1)$ with mean 0 and $\sigma=1$. This function is in $L_{2}(X)$ with probability 1 , since using the orthonormality of the $\phi_{i}$ we can bound its expected norm by

$$
\begin{aligned}
E\left\{\|f\|_{L_{2}(X)}^{2}\right\} & =E\left\{\sum_{i=1}^{\infty} \sum_{j=1}^{\infty} u_{i} u_{j}\left\{\phi_{i} \cdot \phi_{j}\right\}_{L_{2}(X)}\right\} \\
& =\sum_{i=1}^{\infty} \sum_{j=1}^{\infty} E\left\{u_{i} u_{j}\right\}\left\{\phi_{i} \cdot \phi_{j}\right\}_{L_{2}(X)} \\
& =\sum_{i=1}^{\infty} E\left\{u_{i}^{2}\right\}\left\|\phi_{i}\right\|_{L_{2}(X)}^{2}=\sum_{i=1}^{\infty}\left\|\phi_{i}\right\|_{L_{2}(X)}^{2}<\infty
\end{aligned}
$$

where the final inequality follows from theorem 13 . Provided that the norm is a positive function, it follows that the measure of functions not in $L_{2}(X)$ is 0 , as otherwise the expectation would not be finite. However, the function will certainly not be in $\mathcal{F}$ for infinite-dimensional feature spaces. We therefore take the distribution $q$ to be defined over the space $L_{2}(X)$. The covariance function $k_{q}$ is now equal to 


$$
\begin{aligned}
k_{q}(x, z) & =\int_{L_{2}(X)} f(x) f(z) q(f) d f \\
& =\lim _{n \rightarrow \infty} \sum_{i, j=1}^{n} \phi_{i}(x) \phi_{j}(z) \int_{\mathbb{R}^{n}} u_{i} u_{j} \prod_{k=1}^{n}\left(\frac{1}{\sqrt{2 \pi}} \exp \left(-u_{k}^{2} / 2\right) d u_{k}\right) \\
& =\lim _{n \rightarrow \infty} \sum_{i, j=1}^{n} \phi_{i}(x) \phi_{j}(z) \delta_{i j}=\sum_{i=1}^{\infty} \phi_{i}(x) \phi_{i}(z) \\
& =k(x, z) .
\end{aligned}
$$

\subsection{Generative Kernels from Algebraic Statisti- cal Models}

From remark 2 in section 4.4.1, we know that the set of symmetric positive definite matrices is a semi-algebraic set. This remark coupled with the general result that we have shown in section 4.5.4 (i.e. a kernel can be written as a covariance function) sets the basis for the definition of kernels from Algebraic Models (c.f definition 10 in section 4.2). This section presents the three major contributions of this $\mathrm{PhD}$ thesis: the definition of the Quotient Basis Kernel (QBK), the Simplified Fisher kernel and the representation of the Kernels based on the Jensen-Shannon metric in an algebraic context.

\subsubsection{Quotient Basis Kernel}

Definition 35. Design Matrix

Let $\tau$ be a term ordering and let us consider an ordering over the support points $A=\left\{a_{i} \in k^{d}: i=1, \ldots, N\right\}$. Let $L$ be the set of exponents of EST $T_{\tau}$. We call design matrix the following matrix (i.e. the support points evaluated over the elements of $E S T_{\tau}$ ):

$$
Z=\left[a_{i}^{\alpha}\right]_{i=1, \ldots, N, \alpha \in L}
$$

Let us recall the example of the $3 \times 8$ contingency table 4.1 from section 4.2.2. In this example we have calculated the Ideal of this table with the function IdealOfPoints [62] in $\boldsymbol{A p} \boldsymbol{C o} \boldsymbol{C o A}[60,61]$ and the lexicographic order. In our case the ideal is: $\left\langle z^{2}-3 z+2, y^{2}-3 y+2, x^{2}-3 x+2\right\rangle$, and its corresponding Gröbner basis is: $G=\left\{z^{2}-3 z+2, y^{2}-3 y+2, x^{2}-3 x+2\right\}$. Direct application of definition 15 yields the following Quotient Basis: $\operatorname{EST}_{\tau}=\{1, z, y, y z, x, x z, x y, x y z\}$. Now, substitution of the support points from table 4.1 into $\mathrm{EST}_{\tau}$ yields the $8 \times 8$ design matrix:

$$
Z=\left(\begin{array}{llllllll}
1 & 1 & 1 & 1 & 1 & 1 & 1 & 1 \\
1 & 1 & 1 & 1 & 2 & 2 & 2 & 2 \\
1 & 1 & 2 & 2 & 1 & 1 & 2 & 2 \\
1 & 1 & 2 & 2 & 2 & 2 & 4 & 4 \\
1 & 2 & 1 & 2 & 1 & 2 & 1 & 2 \\
1 & 2 & 1 & 2 & 2 & 4 & 2 & 4 \\
1 & 2 & 2 & 4 & 1 & 2 & 2 & 4 \\
1 & 2 & 2 & 4 & 2 & 4 & 4 & 8
\end{array}\right)
$$


Theorem 14. [57]

1. $Z$ is non-singular.

2. Let $e_{i}$ be the d dimensional canonical vector (i.e. with components 0 except in position $i$ where it has value 1$)$. For all $i=1, \ldots, d$ there exists a vector $c_{i} \in k^{d}$ such that $Z c(i)=e_{i}$ and the polynomial $\sum_{\alpha \in L} c_{i_{\alpha}} x^{\alpha}$ interpolates the indicator function of the support point $a_{i}$. That is

$$
\sum_{\alpha \in L} c_{i_{\alpha}} x^{\alpha}=\left\{\begin{array}{cc}
1 & x=a_{i} \\
0 & x \neq a_{i} \text { and } x \in A
\end{array}\right.
$$

Proposition 3. The covariance of $\left.Z \operatorname{cov}(Z)=E(Z-E(Z))(Z-E(Z))^{t}\right)$ is a kernel.

Corollary 1. Quotient Basis Kernel

The covariance of $E S T_{\tau}$ is a kernel.

Proof. The proof is immediate from definitions 30,13 and 4.5.4.

\subsubsection{Fisher Kernel for Exponential Families}

Intuitively, the Fisher Kernel is a function that measures the similarity of two objects on the basis of sets of measurements for each object and a statistical model. In a classification procedure, the class for a new object (whose real class is unknown) can be estimated by minimising, across classes, an average of the Fisher kernel distance from the new object to each known member of the given class.

Let $\mathcal{P}=(P \mid \eta \in N)$ be a regular exponential family with canonical sufficient statistic $T$. If we draw a sample $X_{1}, \ldots, X_{n}$ of independent random vectors from $P_{\eta}$, then, as detailed in section 4.3, the canonical statistic becomes $\sum_{i=1}^{n} T\left(X_{i}\right)=n \bar{T}$ and the log likelihood function takes the form

$$
l(\eta \mid \bar{T})=n\left(\eta^{t} \bar{T}-\phi(\eta)\right)
$$

Definition 36. [57] Score Function

The Score Function is the gradient

$$
U(\bar{T}, \eta)=\frac{\partial l(\eta \mid \bar{T})}{\partial \eta}=n \bar{T}-\frac{\partial}{\partial \eta} \phi(\eta)
$$

By construction of the cumulant generative function $\phi(\eta)$ (c.f. definition 7 from section 4.3), we have $\zeta(\eta)=\frac{\partial}{\partial \eta} \phi(\eta)$, which is the expectation of our regular exponential family.

The information matrix is (minus) the Hessian of the log-likelihood, in this case it is also the Fisher, or expected information, since it does not depend on $X$ :

$$
\operatorname{cov}(U(\bar{T}, \eta))=n \frac{\partial^{2}}{\partial \eta^{2}} \phi(\eta)=E_{\eta}\left\{(n \bar{T}-\zeta(\eta))(n \bar{T}-\zeta(\eta))^{t}\right\}
$$


Definition 37. Fisher Kernel

The Fisher Kernel for a Regular Exponential family is defined as:

$$
k(x, z)=U\left(\bar{T}_{x}, \eta\right) \operatorname{cov}(U(\bar{T}, \eta))^{-1} U\left(\bar{T}_{z}, \eta\right)
$$

Where $T_{x}$ and $T_{z}$ are the sufficient statistics estimated on $x$ and $z$.

In most cases, computation of the Fisher Kernel is computationally expensive so that, normally, the following simplified (practical) Fisher Kernel is implemented

Definition 38. Practical Fisher Kernel

$$
k(x, z)=U\left(\bar{T}_{x}, \eta\right) U\left(\bar{T}_{z}, \eta\right)^{t}
$$

Where $T_{x}$ and $T_{z}$ are the sufficient statistics estimated on $x$ and $z$.

\subsubsection{Kernels based on the Jensen-Shannon metric}

Let $\mathcal{P}=\left(P_{\eta} \mid \eta \in N\right)$ be a regular exponential family with canonical statistic $T$. If we draw a sample of $X_{1}, \ldots, X_{n}$ independent random vectors from $P_{\eta}$, then the canonical statistic becomes $n \hat{T}=\sum_{i=1}^{n} X_{i}$ and the log-likelihood takes the form

$$
l(\eta \mid \hat{T})=n\left[\eta^{t} T-G(\eta)\right]
$$

For maximum likelihood estimation on a Regular Exponential Family $P_{M}=$ $\left(P_{\eta}, \eta \in M\right), M \subseteq N$ we need to maximize $l(\eta \mid \hat{T})$ over the set $M$. Let $A$ and $g$ be the semi-algebraic set and the diffeomorfism that define the parameter space $M$. Let $I(A)=\left(f_{1}, \ldots, f_{m}\right)$ be the ideal of model invariants and let $\gamma=g(\eta)$ the parameters after re-parametrization by $g$ [58]. Then, the maximization problem can be relaxed to

$$
\begin{aligned}
& \max l(\gamma \mid \hat{T}) \\
& \text { s.t. } f_{i}=0 \quad i=1, \ldots, m,
\end{aligned}
$$

where $l(\gamma \mid \hat{T})=g^{-1}(\gamma)^{t} \hat{T}-G\left(g(\gamma)^{-1}\right)$. In our case, we work with the probability simplex as a semi-algebraic set [58] for discrete random variables, which is a convex polyhedron in any dimension. Therefore, the optimization problem (4.82) is convex. It is important to note that this algebraic representation agrees with the standard theory and it can be represented as a Bregman Divergence as we will show below.

Let $F$ be the convex-dual in the Legendre sense of the partition function $G$. A Bregman Divergence is defined as:

Definition 39. Bregman Divergence

$$
\begin{aligned}
B_{F}\left(\hat{T}|| \nabla G\left(g^{-1}\left(\gamma_{i}\right)\right)\right)= & F(\hat{T})-F\left(\nabla G\left(g^{-1}\left(\gamma_{i}\right)\right)\right. \\
& -\nabla F\left(\nabla G\left(g^{-1}\left(\gamma_{i}\right)\right)\right) \cdot\left(\hat{T}-\nabla G\left(g^{-1}\left(\gamma_{i}\right)\right)\right) .
\end{aligned}
$$

By the Legendre dual we have

$$
F\left(\nabla G\left(g^{-1}(\gamma)\right)=\nabla G\left(g^{-1}(\gamma)\right) g^{-1}(\gamma)-G\left(g^{-1}(\gamma)\right)\right.
$$


Also, $F$ and $G$ are Legendre functions if their derivatives are inverse functions of each other (i.e. $\nabla F\left(\nabla G\left(g^{-1}(\gamma)\right)=g^{-1}(\gamma)\right.$ ). Since $F(\hat{T})$ does not depend on the parametrization, our optimization problem becomes:

$$
\begin{aligned}
& \max l(\gamma \mid \hat{T})=\max \left\{F(\hat{T})-\sum_{i=1}^{m} B_{F}\left(\hat{T}|| \nabla G\left(g^{-1}\left(\gamma_{i}\right)\right)\right\}\right. \\
& =\min \left\{\sum_{i=1}^{m} B_{F}\left(\hat{T}|| \nabla G\left(g^{-1}\left(\gamma_{i}\right)\right)\right\}\right. \\
& \text { s.t. } f_{i}=0 \quad i=1, \ldots, m
\end{aligned}
$$

In this respect, we can apply the idea that given new facts $x_{k}$, a new distribution parametrized by $\eta_{i}$ should be chosen which is as hard to discriminate from the original parametrization $\eta$ as possible so that the new data produces as small an information gain in $K L\left(\eta_{i} \| \eta\right)$ or $B_{F}\left(\hat{T} \| \nabla G\left(g^{-1}\left(\gamma_{i}\right)\right)\right.$ as small as possible ${ }^{5}$. In other words, what we want to achieve is the minimum of the cross-entropy (i.e. second term in equation A.8). This approach was already exploited by Kullback and Leibler in [80] and termed it Principle of Minimum Discrimination Information (MDI).

Therefore, it is now natural to use the Jensen-Shannon Divergence ${ }^{6}$ (c.f. equation A.11) as a metric in order to build kernels that exploit the generative properties of the data. As opposed to [76], the main contribution here is that we are bridging together the use of semi-algebraic sets (which are needed for the parametrization) and the dual structure induced by the diffeomorfism $g$ that re-parametrises the optimization problem.

Now we only have to apply the Jensen-Shannon metric over the dual space of functions and the propositions of section 4.5.2. More specifically,

Definition 40. Let $\gamma_{1}, \gamma_{2} \in M$, by equation A.10:

$$
J S\left(\gamma_{1}, \gamma_{2}\right)=\frac{F\left(\gamma_{1}\right)+F\left(\gamma_{2}\right)}{2}-F\left(\frac{\gamma_{1}+\gamma_{2}}{2}\right) .
$$

Proposition 4. [76, 77, 78] Centred Kernel

By property 8 and definition 40 , let $x_{0} \in X$ define the centred kernel as $\phi: X \times X \rightarrow \mathbb{R}$

$$
\phi(x, y)=J S\left(x, x_{0}\right)+J S\left(y, x_{0}\right)-J S(x, y)-J S\left(x_{0}, x_{0}\right) .
$$

Proposition 5. [76, 77, 78] Exponentiated Kernel

By property 9 and definition 40, we define the exponentiated kernel as $\phi$ : $X \times X \rightarrow \mathbb{R}$

$$
\phi(x, y)=\exp (-t J S(x, y))
$$

$\forall t>0$.

Proposition 6. [76, 77, 78] Inverse Kernel

By proposition 10 and definition 40, we define the inverse kernel as $\phi$ : $X \times X \rightarrow \mathbb{R}$

$$
\phi(x, y)=\frac{1}{t+J S(x, y)}
$$

$\forall t>0$.

\footnotetext{
${ }^{5} \mathrm{KL}$ is a Bregman Divergence

${ }^{6}$ remember that the KL is not a metric
} 


\section{Chapter 5}

\section{Background: Methods for Regression, Classification and Dimensionality Reduction}

If it's subject to rules, it can be learned!

Maty Tchey

In this section we present the required background about Regression, Classification and Dimensionality Reduction techniques that are used in this PhD. thesis (chapters 6, 7 and 8). In particular we focus on Classification and Regression Trees (used in Chapter 6), Logistic Regression (widely used by medical community), Dimensionality Reduction Techniques like Factor Analysis (used in chapter 7), Ridge Regression and the RVM (chapter 8). Here we also present the SVM (chapter 8) where we will deploy the Kernels proposed in Chapter 4.

\subsection{Regression Trees}

In regression trees $[81,82]$, our learning sample $L$ consists of $N$ inputs $x_{i}$ where $x \in X$ and a response $y_{i}$ where $y \in \mathbb{R}$. Therefore, we want to predict the response $r(x)$ from the learning sample $L$ such that

$$
\begin{aligned}
\mathbb{R}^{N} & \rightarrow \mathbb{R} \\
x \rightarrow y & =r(x) \quad x_{i} \in X .
\end{aligned}
$$

We have $N$ observations $\left(x_{i}, y_{i}\right)$ for $i=1, \ldots, N$ with $x_{i}=\left(x_{i_{1}}, \ldots, x_{i_{m}}\right)$. Assume that we have partitioned our data into $M$ regions $R_{1}, R_{2}, \ldots, R_{M}$, which yield the same result $c_{m}$ and that the response is given by the sum of the responses over the whole region:

$$
r(x)=\sum_{m=1}^{M} c_{m} I\left(x \in R_{m}\right) .
$$


Here, $I\left(x \in R_{m}\right)$ is the index function, which returns one if $x \in R_{m}$ and 0 otherwise. Defining the cost function as the sum of squares

$$
J=\sum\left(y_{i}-x_{i}\right)^{2},
$$

it can be shown that the best $\hat{c}_{m}$ is just the expectation of $y_{i}$ in region $R_{m}$

$$
\hat{c}_{m}=E\left\{y_{i} \mid x_{i} \in R_{m}\right\} .
$$

The best split at each level of tree branching is normally found by means of a greedy algorithm, which starts with the complete data sample and splits the $j^{t h}$ variable at split point $s$, so that the following two half-regions are defined:

$$
\begin{aligned}
& R_{1}(j, s)=\left\{X \mid X_{j} \leq s\right\} \\
& R_{2}(j, s)=\left\{X \mid X_{j}>s\right\},
\end{aligned}
$$

We now have to find the splitting variable $j$ and split point $s$ that solve the expression:

$$
\min _{j, s}\left(\min _{c_{1}} \sum_{x_{i} \in R_{1}(j, s)}\left(y_{i}-c_{1}\right)^{2}+\min _{c_{2}} \sum_{x_{i} \in R_{2}(j, s)}\left(y_{i}-c_{2}\right)^{2}\right) .
$$

Thus, for any $j, s$ pair, the inner minimization is solved by:

$$
\begin{aligned}
& \hat{c}_{1}=E\left\{y_{i} \mid x_{i} \in R_{1}(j, s)\right\} \\
& \hat{c}_{2}=E\left\{y_{i} \mid x_{i} \in R_{2}(j, s)\right\} .
\end{aligned}
$$

Commonly, when defining regression trees over a large number of variables, a large tree $T_{0}$ is grown, stopping the splitting process outlined above when a minimum node size is reached. In order to avoid data over fitting, this large tree is reduced through a cost-complexity pruning process [81]. Let us define a sub tree $T \subset T_{0}$ as any tree that can be obtained by pruning $T_{0}$. Let us also index the terminal nodes by $k$, with node $k$ representing the splitting region $R_{k}$ and $|T|$ as the number of terminal nodes in $T$. This way we can provide an expression for the estimation $\hat{c}_{m}$. Defining $N_{k}$ as the number of cases in region $R_{k}$ and the tree cost function $Q_{k}(T)$, we have:

$$
\begin{gathered}
\hat{c}_{k}=\frac{1}{N_{k}} \sum_{x_{i} \in R_{k}} y_{i}, \\
Q_{k}(T)=\frac{1}{N_{k}} \sum_{x_{i} \in R_{k}}\left(y_{i}-\hat{c}_{k}\right)^{2},
\end{gathered}
$$

By adding an adjustment coefficient alpha, the cost complexity criterion becomes

$$
J_{\alpha}(T)=\sum_{k=1}^{|T|} N_{k} Q_{k}(T)+\alpha|T| .
$$

What we want to obtain is the sub tree $T \subset T_{0}$ that minimizes $J_{\alpha}(T)$ for each $\alpha$. With this approach, for each $\alpha$ there is a unique smallest sub tree $T_{\alpha}$ that minimizes $J_{\alpha}(T)$. This $T_{\alpha}$ is found by means successively collapsing the internal nodes that produce the smallest per-node increase in $\sum_{k=1}^{|T|} N_{k} Q_{k}(T)$, and continue until we produce a root tree (i.e. a tree with no parent nodes). 


\subsection{Classification Techniques}

\subsubsection{Logistic Regression: Classification as Binomial Re- gression}

Logistic regression studies binomially distributed variables of the form $C_{i} \sim$ $B\left(n_{i}, p_{i}\right)$ where $n_{i}$ and $p_{i}$ correspond to the number of patients and the probability of exitus. In our study, $C_{i}$ is a class label that takes the value 1 for survival and 0 for exitus. The logistic model proposes that, for each patient $i$, there is a set of explanatory variables that might inform the final probability. Thus, the model takes the form: $p_{i}=E\left(\frac{C_{i}}{n_{i}} \mid X_{i}\right)$, for each variable $i$ (be it from the original set of variables listed in Table 7.2 , or one of the extracted factors).

Here, the natural logs of the odds ratio for the unknown binomial probabilities are modelled as a linear function of $X_{i}$ :

$$
\log \left(\frac{p_{i}}{1-p_{i}}\right)=\beta_{0}+B^{T} \cdot X_{i},
$$

where $\beta_{0}$ is the intercept and $B$ is the vector of logistic regression coefficients. In this thesis, the intercept and regression coefficients were estimated by ML with a generalized linear model.

\subsubsection{Support Vector Machines}

We have $L$ training points, where each input $x_{i}$ has $D$ attributes (i.e. dimensionality $D$ ) and is one of the two classes $y_{i}=+1$ or $y_{i}=-1$. In other words, our training data is of the form $\left\{x_{i}, y_{i}\right\}$ where $i=1, \ldots, L, y_{i} \in\{-1,1\}$ and $\mathbf{x} \in \mathbb{R}^{D}$. For now, let us assume that we can draw a hyperplane separating the $\mathbf{x}_{1}, \ldots, \mathbf{x}_{L}$ in two disjoint sets corresponding to the training labels $y_{i}=1$ and $y_{i}=-1$.

The general equation of this hyperplane is $\mathbf{w} \mathbf{x}+b=0$. Of course:

- $\mathbf{w}$ is normal to the hyperplane.

- $\frac{b}{\mathbf{w}}$ is the perpendicular distance from the hyperplane to the origin.

Support vectors are the examples closest to the separating hyperplane and the aim of Support Vector Machines (SVM) is to orientate this hyperplane in a way that is as far as possible from the closest members of both classes $[73,71,83,84]$. Therefore, SVM is equivalent to selecting the variables $\mathbf{w}$ and $b$ so that our training data can be described as:

$$
y_{i}\left(\mathbf{x}_{\mathbf{i}} \mathbf{w}+b-1\right) \geq 0 \forall i .
$$

Considering just the points closest to the separating hyperplane (i.e. the Support Vectors), then the two planes $H_{1}$ and $H_{2}$ where these points lie on are:

$$
\begin{array}{cc}
\mathbf{x}_{\mathbf{i}} \mathbf{w}+b=1 & \text { for } H_{1} \\
\mathbf{x}_{\mathbf{i}} \mathbf{w}+b=-1 & \text { for } H_{2}
\end{array}
$$

Defining as $d_{1}$ the distance from $H_{1}$ to the hyperplane and $d_{2}$ from $H_{2}$ to it. The hyperplane's equidistance to $H_{1}$ and $H_{2}$ means that $d_{1}=d_{2}-c$ a quantity 


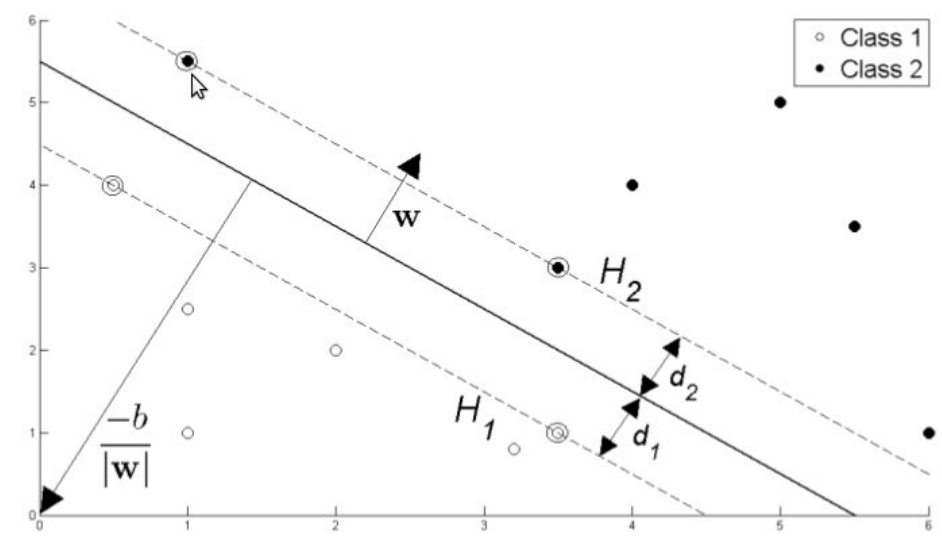

Figure 5.1: Hyperplane through two linearly separable classes.

known as the SVM margin. Since we want to orientate the hyperplane as far as possible to the Support Vectors, we need to maximize this margin.

It is easy to show that this margin is $1 /\|\mathbf{w}\|$ so that our optimization problem becomes:

$$
\begin{aligned}
& \max \left(\frac{1}{\|\mathbf{w}\|}\right)=\min (\|\mathbf{w}\|) \\
& \text { s.t } y_{i}\left(\mathbf{x}_{\mathbf{i}} \mathbf{w}+b\right)-1 \geq 0
\end{aligned}
$$

Minimizing $\|\mathbf{w}\|$ is equivalent to minimizing $\frac{1}{2}\|\mathbf{w}\|^{2}$ ) and the use of this term makes it possible to use Quadratic Programming (QP) optimization. Therefore,

$$
\begin{gathered}
\min \left(\frac{1}{2}\|\mathbf{w}\|^{2}\right) \\
\text { s.t } y_{i}\left(\mathbf{x}_{\mathbf{i}} \mathbf{w}+b\right)-1 \geq 0
\end{gathered}
$$

The optimization problem in equation 5.14 is minimized by means of Lagrange multipliers $\alpha$.

$$
\begin{aligned}
& \mathcal{L}_{p}=\frac{1}{2}\|\mathbf{w}\|^{2}-\alpha\left(y_{i}\left(\mathbf{x}_{\mathbf{i}} \mathbf{w}+b\right)\right.-1 \forall i) \\
&=\frac{1}{2}\|\mathbf{w}\|^{2}-\sum_{i=1}^{L} \alpha_{i}\left(y_{i}\left(\mathbf{x}_{\mathbf{i}} \mathbf{w}+b\right)-1\right) \\
&=\frac{1}{2}\|\mathbf{w}\|^{2}-\sum_{i=1}^{L} \alpha_{i}\left(y_{i}\left(\mathbf{x}_{\mathbf{i}} \mathbf{w}+b\right)\right)+\sum_{i=1}^{L} \alpha_{i}
\end{aligned}
$$

Differentiating $\mathcal{L}_{p}$ with respect to $\mathbf{w}$ and $b$ and setting the derivatives to zeros

$$
\begin{array}{ll}
\frac{\partial}{\partial \mathbf{w}} \mathcal{L}_{p}=0 & \mathbf{w}=\sum_{i=1}^{L} \alpha_{i} y_{i} \mathbf{x}_{\mathbf{i}} \\
\frac{\partial}{\partial \mathbf{b}} \mathcal{L}_{p}=0 & \sum_{i=1}^{L} \alpha_{i} y_{i}=0 .
\end{array}
$$


Substitution into equation 5.15 gives a new formulation which being dependent on $\alpha$; now we need to maximize

$$
\begin{gathered}
\mathcal{L}_{d}=\sum_{i=1}^{L} \alpha_{i}-\frac{1}{2} \sum_{i=1}^{L} \sum_{j=1}^{L} \alpha_{i} \alpha_{j} y_{i} y_{j} \mathbf{x}_{\mathbf{i}} \mathbf{x}_{\mathbf{j}} \text { s.t } \alpha_{i} \geq 0 \forall i, \sum_{i=1}^{L} \alpha_{i} y_{i}=0 \\
=\sum_{i=1}^{L} \alpha_{i}-\frac{1}{2} \sum_{i=1}^{L} \sum_{j=1}^{L} \alpha_{i} H_{i j} \alpha_{j} \text { where } H_{i j}=y_{i} y_{j} \mathbf{x}_{\mathbf{i}} \mathbf{x}_{\mathbf{j}} \\
=\sum_{i=1}^{L} \alpha_{i}-\frac{1}{2} \alpha^{t} \mathbf{H} \alpha \text { s.t } \alpha_{i} \geq 0 \forall i, \sum_{i=1}^{N} \alpha_{i} y_{i}=0
\end{gathered}
$$

This formulation of the optimization problem is referred as the Dual form of the Primary $\mathcal{L}_{p}$. It is important to note that this dual form only requires the calculation of the scalar product of each input vector $\mathbf{x}_{\mathbf{i}}$. This is very important for the Kernel Trick.

Now we have moved from minimizing $\mathcal{L}_{p}$ to maximizing $\mathcal{L}_{d}$, so we need to find:

$$
\begin{gathered}
\arg \max _{\alpha} \sum_{i=1}^{L} \alpha_{i}-\frac{1}{2} \alpha^{t} \mathbf{H} \alpha \\
\text { s.t } \alpha_{i} \geq 0 \forall i \text { and } \sum_{i=1}^{L} \alpha_{i} y_{i}=0 .
\end{gathered}
$$

This is a convex quadratic optimization problem, running a QP solver (in our case the Matlab QP solver) will return w. Now we have to calculate $b$.

Any data point satisfying the equation 5.16, which is a Support Vector $x_{s}$ will have the formal

$$
y_{s}\left(\mathbf{x}_{\mathbf{s}} \mathbf{w}+b\right)=1
$$

and

$$
y_{s}\left(m \in S \alpha_{m} y_{m} \mathbf{x}_{\mathbf{m}} x_{s}+b\right)=1
$$

where $S$ denotes the set of indices of Support Vectors. $S$ is determined by finding the indices $i$ where $\alpha_{i}>0$. Multiplying through by $y_{s}$ and then using $y_{s}^{2}=1$ from 5.11

$$
\begin{gathered}
y_{s}^{2}\left(\sum_{m \in S} \alpha_{m} y_{m} \mathbf{x}_{m} \mathbf{x}_{s}+b\right)=y_{s} \\
b=y_{s}-\sum_{m \in S} \alpha_{m} y_{m} \mathbf{x}_{m} x_{s} .
\end{gathered}
$$

Also, instead of using an arbitrary Support Vector $\mathbf{x}_{s}$, it is better to take an average over all the support vectors in $S$

$$
b=\frac{1}{N} \sum_{s \in S}\left(y_{s}-\sum_{m \in S} \alpha_{m} y_{m} \mathbf{x}_{\mathbf{m}} \mathbf{x}_{\mathbf{s}}\right)
$$


Now we have the variables $\mathbf{w}$ and $b$ that define our separating hyperplane's optimal orientation and hence our first simple Support Vector Machine.

The Support Vector Machine for solving a linearly separable binary classification problem is done as follows:

1. Calculate $\mathbf{H}$ where $H_{i j}=y_{i} y_{j} \mathbf{x}_{\mathbf{i}} \mathbf{x}_{\mathbf{j}}$.

2. Solve the optimization problem 5.17 using a QP solver.

3. Calculate $\mathbf{w}=\sum_{i=1}^{L} \alpha_{i} y_{i} \mathbf{x}_{\mathbf{i}}$.

4. Determine the set of Support Vectors $S$ by finding the indices such that $\alpha_{i}>0$.

5. Calculate $b$ through equation 5.19 .

6. Each new point $\mathbf{x}^{\prime}$ is classified by evaluating $y^{\prime}=\operatorname{sgn}\left\{\mathbf{w} \mathbf{x}^{\prime}+b\right\}$.

Unfortunately, the application of the theory outlined above is not sufficient to tackle real life problems where data is not fully linearly separable. This issue can be both overcome by means of augmenting the dimensionality of our input space by means of a Kernel transformation and also by relaxing the constraints in 5.11 by allowing the presence of misclassified points. This is done by introducing a positive slack variable $\xi_{i} i=1, \ldots, L$

$$
y_{i}\left(\mathbf{x}_{\mathbf{i}} \mathbf{w}+b\right)-1+\xi_{i} \geq 0 \text { where } \xi_{i} \geq 0 \forall i .
$$

This is the Soft Margin SVM where the points falling on the incorrect side of the margin boundary have a penalty that increases with the distance from it. Since our goal now is also to reduce the number of misclassified points, it is sensible to adapt our function 5.14 to find:

$$
\begin{gathered}
\min \frac{1}{2}\|\mathbf{w}\|^{2}+C \sum_{i=1}^{L} \xi_{i} \\
\text { s.t } y_{i}\left(\mathbf{x}_{\mathbf{i}} \mathbf{w}\right)-1+\xi_{i} \geq 0 \forall i
\end{gathered}
$$

The parameter $C$ controls the trade-off between the slack variable penalty and the size of the margin. Again, reformulating as a Lagrangian, which as before we need to minimize w.r.t $\mathbf{w}, b$ and $\xi_{i}$ and maximize w.r.t $\alpha$ (where $\alpha_{i} \geq 0, \mu_{i} \geq 0 \forall i$ :

$$
\mathcal{L}_{p}=\frac{1}{2}\|\mathbf{w}\|^{2}+C \sum_{i=1}^{L} \xi_{i}-\sum_{i=1}^{L} \alpha_{i}\left(y_{i}\left(\mathbf{x}_{\mathbf{i}} \mathbf{w}+b\right)-1+\xi_{i}\right)-\sum_{i=1}^{L} \mu_{i} \xi_{i}
$$

Differentiating w.r.t $\mathbf{w}, b$ and $\xi_{i}$ and setting the derivatives to zero:

$$
\begin{array}{cc}
\frac{\partial}{\partial \mathbf{w}} \mathcal{L}_{p}=0 & \mathbf{w}=\sum_{i=1} L \alpha_{i} y_{i} \mathbf{x}_{\mathbf{i}} \\
\frac{\partial}{\partial b} \mathcal{L}_{p}=0 & \sum_{i=1}^{L} \alpha_{i} y_{i}=0 \\
\frac{\partial}{\partial \xi_{i}} \mathcal{L}_{p}=0 & C=\alpha_{i}+\mu_{i} .
\end{array}
$$


Substitution of these in $\mathcal{L}_{d}$ has the same form as equation 5.17. However, the last equation in 5.23 together with $\mu_{i} \geq 0 \forall i$ implies that $\alpha \leq C$. We therefore need to finding

$$
\begin{gathered}
\arg \max _{\alpha}\left(\sum_{i=1}^{L} \alpha_{i}-\frac{1}{2} \alpha^{t} \mathbf{H} \alpha\right) \\
\text { s.t. } 0<\alpha_{i}<C \text { and } \sum_{i=1}^{L} \alpha_{i} y_{i}=0 .
\end{gathered}
$$

Now $b$ is calculated in the same way as before, though in this instance the set of Support Vectors used to calculate $b$ is determined by finding the indices $i$ where $0<\alpha_{i}<C$.

The Soft Margin Support Vector Machine is applied as follows:

1. Calculate $\mathbf{H}$ where $H_{i j}=y_{i} y_{j} \mathbf{x}_{\mathbf{i}} \mathbf{x}_{\mathbf{j}}$.

2. Choose an appropriate value for $\mathrm{C}$ (for small problems this can be done by means of a grid search).

3. Solve the optimization problem 5.24 using a QP solver.

4. Calculate $\mathbf{w}=\sum_{i=1}^{L} \alpha_{i} y_{i} \mathbf{x}_{\mathbf{i}}$

5. Determine the set of Support Vectors $S$ by finding the indices such that $0<\alpha_{i}<C$.

6. Calculate $b$ through equation 5.19 .

7. Each new point $\mathbf{x}^{\prime}$ is classified by evaluating $y^{\prime}=\operatorname{sgn}\left\{\mathbf{w} \mathbf{x}^{\prime}+b\right\}$.

So far we have only tackled linearly separable data and we started our algorithms by creating a matrix $\mathbf{H}$ from the dot product of our input variables

$$
H_{i j}=y_{i} y_{j} \mathbf{x}_{\mathbf{i}} \mathbf{x}_{\mathbf{j}}
$$

The SVM is easily extended to the Non-linear case just by replacing the linear dot product $\mathbf{x}_{\mathbf{i}} \mathbf{x}_{\mathbf{j}}$ by any suitable kernel like the Quotient Basis Kernel or the Simplified Fisher kernel proposed in this $\mathrm{PhD}$ thesis.

\subsubsection{Classification with Feature Selection: Relevance Vec- tor Machines}

The general regression problem posed by RVM can be written as $[73,85,86]$ :

$$
y=w^{t} \psi(x),
$$

where $\psi(x)$ is a basis function. In order to estimate the weights $w$ from our training examples, it is assumed that each target $t_{i}$ in the training sample (valued 1 for survival and -1 for exitus in the current study) represents the true model $y_{i}$ contaminated by i.i.d Gaussian noise $\epsilon_{i} \sim N\left(0, \sigma^{2}\right)$, so that, $\forall i$ :

$$
t_{i}=w^{t} \psi\left(x_{i}\right)+\epsilon_{i}
$$

Therefore, 


$$
\begin{gathered}
p\left(t_{i} \mid x_{i}, w, \sigma^{2}\right) \sim N\left(y_{i}, \sigma^{2}\right)= \\
\frac{1}{\sqrt{2 \pi \sigma^{2}}} \exp \left(\frac{1}{2 \sigma^{2}}\left(t_{i}-w^{t} \psi\left(x_{i}\right)\right)^{2}\right)
\end{gathered}
$$

For the $N$ training points,

$$
\begin{gathered}
p\left(t \mid x_{i}, w, \sigma^{2}\right)=\prod_{i=1}^{N} N\left(w^{t} \psi\left(x_{i}\right), \sigma^{2}\right)= \\
\frac{1}{\left(2 \pi \sigma^{2}\right)^{N / 2}} \exp \left(\frac{1}{2 \sigma^{2}}\|t-\Psi w\|\right),
\end{gathered}
$$

where $t$ is the vector of training targets $t_{i}$, and the $N \times M$ matrix $\Psi$ is built so that the $i^{\text {th }}$ row represents vector $\psi\left(x_{i}\right)$.

The growth of the weights $w$ can be constrained by defining an explicit prior probability distribution on $w$. Assuming a Gaussian distribution on $w$, and defining $S=s I$ as the hyper-parameter matrix where $I$ is $N \times N$ identity matrix and $S=\left[s_{1}, \ldots, s_{N}\right]$ is a vector where each $s_{i}$ describes the inverse variance for each $w_{i}$.

The posterior probability over the unknown parameters is defined as:

$$
\begin{gathered}
p\left(w, s, \sigma^{2} \mid t\right)=p\left(w \mid t, s, \sigma^{2}\right) p\left(s, \sigma^{2} \mid t\right) \\
p\left(w \mid t, s, \sigma^{2}\right)=\frac{|\Sigma|^{1 / 2}}{(2 \pi)^{N / 2}} \exp \left(\frac{-1}{2}(w-\mu)^{t} \Sigma^{-1}(w-\mu)\right),
\end{gathered}
$$

where $\Sigma=\left(\frac{1}{\sigma^{2}} \Psi^{t} \Psi+S\right)^{-1}$ and $\mu=\frac{1}{\sigma^{2}} \Sigma \Psi t$. To estimate $\mu$ and $\Sigma$, we need to maximize the evidence:

$$
p\left(t \mid s, \sigma^{2}\right)=\int p\left(t \mid w, \sigma^{-2}\right) p(w \mid s) d w
$$

Assuming uniform hyperpriors and expanding eq.5.31, it is possible to calculate the following marginal likelihood function:

$$
\begin{gathered}
\ln p\left(t \mid s, \sigma^{-2}\right)=\frac{1}{2} \sum_{i=1}^{M} \ln s_{i}-\frac{N}{2}\left(\ln \sigma^{-2}+\ln (2 \pi)\right) \\
-\frac{1}{2}\left(\sigma^{-2} t^{t} t-\mu^{t} \Sigma^{-1} \mu+\ln |\Sigma|\right),
\end{gathered}
$$

which has to be maximized w.r.t. $\sigma^{-2}$ and $s$.

It is important to note that during the iterative process associated to the maximization of the expression in eq. 5.32, some $s_{i}$ may tend towards infinity, which entails $\lim _{s_{i} \rightarrow \infty} \Sigma=0$ and $\lim _{s_{i} \rightarrow \infty} \mu=0$. In this situation, some $w_{i}$ will take values close to zero, which means that the adaptive effect of the hyperparameters will effectively switch off those input features that are deemed to be irrelevant for the prediction. This is, in fact, a form of soft feature selection, or, more precisely, a form of automatic relevance determination.with inputs corresponding to weights different from zero shall be called relevance vectors. 


\subsection{Dimensionality Reduction}

\subsubsection{Feature Selection Methods}

\section{Ridge Regression}

Ridge regression shrinks the regression coefficients by imposing a penalty on their size. More particularly, the ridge coefficients are obtained by minimizing a penalized sum of squares $[81,79]$ of the form:

$$
\min _{\mathbf{w}} \mathcal{L}_{\lambda}(\mathbf{w}, T)=\min _{\mathbf{w}} \lambda\|\mathbf{w}\|^{2}+\sum_{i=1}^{M}\left(y_{i}-g\left(\mathbf{x}_{\mathbf{i}}\right)\right)^{2},
$$

for a prediction function of the form $g(\mathbf{x})=\langle\mathbf{w}, \mathbf{x}\rangle$ and training set $T$. Here $\lambda$ is a positive number that defines the relative trade-off between norm and loss and hence controls the degree of shrinkage. Taking the derivative of the loss function with respect to the parameters we obtain:

$$
\mathbf{X}^{\mathbf{t}} \mathbf{X} \mathbf{w}+\lambda \mathbf{w}=\left(\mathbf{X}^{\mathbf{t}} \mathbf{X}+\lambda \mathbf{I}\right) \mathbf{w}=\mathbf{X}^{\mathbf{t}} \mathbf{y}
$$

Again, $\mathbf{I}$ is the $N \times N$ identity matrix. In this case, the matrix $\left(\mathbf{X}^{\mathbf{t}} \mathbf{X}+\lambda \mathbf{I}\right)$ is always invertible if $\lambda>0$ so that the solution is given by:

$$
\mathbf{w}=\left(\mathbf{X}^{\mathbf{t}} \mathbf{X}+\lambda \mathbf{I}\right)^{-1} \mathbf{X}^{\mathbf{t}} \mathbf{y}
$$

\section{The Lasso}

If instead of using the $L_{2}(X)$ penalty term of equation 5.33, we use an $L_{1}(X)$, what we obtain is a quadratic programming convex problem with linear constraints more generally known as the Lasso. More particularly, the cost function to minimize has the form:

$$
\min _{\mathbf{w}} \mathcal{L}_{\lambda}(\mathbf{w}, T)=\min _{\mathbf{w}} \lambda|\mathbf{w}|_{1}+\sum_{i=1}^{M}\left(y_{i}-g\left(\mathbf{x}_{\mathbf{i}}\right)\right)^{2},
$$

for a prediction function of the form $g(\mathbf{x})=\langle\mathbf{w}, \mathbf{x}\rangle$ and training set $T$. Again, $\lambda$ is a positive number that defines the relative trade-off between norm and loss and hence controls the degree of shrinkage. Of course, the use of a L1 penalty turns the solutions non-linear in $\mathbf{y}$ and a quadratic programming algorithm is needed to compute them (for example, throughout this work we have used Matlab QP solver).

\subsection{Feature Extraction Methods}

Out of the broad palette of existing feature extraction methods, some of the most widely-used ones are Principal Component Analysis (PCA) [87], Non-Negative Matrix Factorization (NMF) [88], and Factor Analysis (FA) [89]. PCA obtains new factors using the eigenvectors of the sample covariance matrix. This matrix presents the property that a sub-base made of the eigenvectors associated with the highest eigenvalues yields a reconstruction that minimizes the square error. 
NMF is also a natural way of obtaining a meaningful base because the observations are all positive, and most follow a multinomial distribution. Provided that this factorization does not give a ranking of the elements of the base as in the case of PCA, an arbitrary dimension of the sub-base that spans the observation can be selected. The bases (factors) that are obtained with both methods span a subspace which reconstructs the original observation with an error.

The covariance matrix can be decomposed into the sum of two terms: the product of the base that we use in order to represent the observed data, plus an error term, in the form $\Sigma=\Lambda \Lambda^{T}+\Psi$. In PCA and NMF, the covariance of the error term is a full matrix, which means that the factor base does not account for all the interactions between the observed variables. In other words, the error term still contains information about interactions or relations between these variables in addition to the specific information of each variable (diagonal term of $\Psi)$.

To overcome this limitation, we propose the use of FA, which finds a decomposition of the covariance matrix $\Sigma=\Lambda \Lambda^{T}+\Psi$ such that $\Psi$ is a diagonal matrix. This method selects the factors following a criterion based on the correlation between features of the observation vector. In our implementation, the model is estimated using maximum likelihood (ML), which explicitly assumes a Gaussian distribution for $x$. Nevertheless, and independently of assumptions concerning data distribution, ML searches for a decomposition of $\Sigma$ so that the error matrix $\Psi$ has a diagonal structure. Therefore, the model generates the observation from a set of latent variables that are independent of the error term, and takes into account all the correlations between variables.

The following two sections show that, although the observed variables in the analysed data fail to pass a multivariate normality test, the covariance matrix of the residual error can be assumed to be diagonal.

\section{Factor Analysis Through Statistical Algebra}

$[68,72]$ Factor Analysis (FA) concerns a Gaussian hidden variable model with $d$ observed variables $X_{i}$, where $i \in[d]=\{1, \ldots, d\}$, and $k$ hidden variables $Y_{j}$, where $j \in[k]=\{1, \ldots, k\}$. FA assumes that $(X, Y)$ follows a joint multivariate normal distribution with positive definite covariance matrix. The FA model $\mathbf{F}_{d, k}$ is defined by the requirement that the observed variables $X_{i}, i \in[d]$, are conditionally independent given the hidden variables $Y_{j}, j \in[k]$. This FA model can be visualized using the graphical model formalism outlined in section 4.4.3, in which the dependence structure between observed data and hidden variables is encoded by a DAG. This directed graph has the vertex set $\left\{X_{1}, \ldots, X_{d}, Y_{1}, \ldots, Y_{k}\right\}$, and the edges are $Y_{i} \rightarrow X_{i}$ for all $j \in[k]$ and $i \in[d]$, as shown in figure 5.2

Proposition 7. [72] The FA model $\mathbf{F}_{\mathbf{d}, \mathbf{k}}$ is the family of multivariate normal distributions $N_{d}(\mu, \Sigma)$ on $\mathbb{R}^{d}$ whose mean vector $\mu$ is an arbitrary vector in $\mathbb{R}^{d}$ and whose covariance matrix $\Sigma$ lies in the (non-convex) cone

$$
\begin{gathered}
\mathbf{F}_{\mathbf{d}, \mathbf{k}}=\left\{\Omega+\Lambda \Lambda^{t} \in \mathbb{R}^{d \times d}: \Omega \succ 0 \text { diagonal, } \Lambda \in \mathbb{R}^{d \times k}\right\} \\
=\left\{\Omega+\Psi \in \mathbb{R}^{d \times d}: \Omega \succ 0 \text { diagonal, } \Psi \succeq 0 \text { symmetric, rank }(\Psi) \leq k\right\} .
\end{gathered}
$$




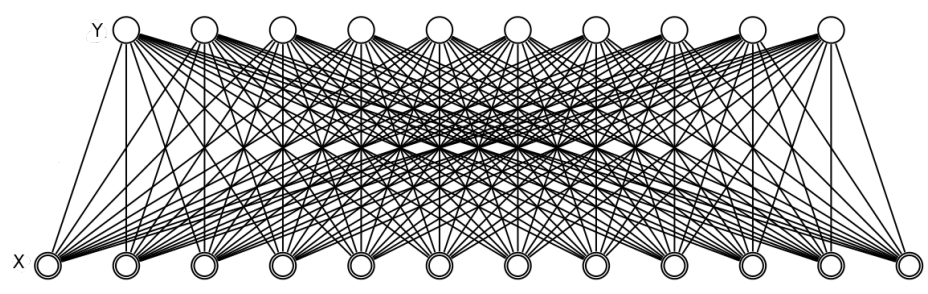

Figure 5.2: Graphical Representation of the Factor Analysis Model $\mathbf{F}_{\mathbf{1 2 , 1 0}}$

Here $A \succ 0$ means that $A$ is a positive definite matrix and $A \succeq 0$ means that matrix $A$ is positive semi-definite. By proposition 7 , the semi-algebraic set $\mathbf{F}_{\mathbf{d}, \mathbf{k}}$ can be parametrized by the polynomial map with coordinates:

$$
\sigma_{i j}=\left\{\begin{array}{c}
\omega_{i i}+\sum_{r=1}^{k} \lambda_{i r}^{2} \text { if } i=j \\
\sum_{r=1}^{k} \lambda_{i r} \lambda_{j r} \text { if } i<j,
\end{array}\right.
$$

where $\omega_{i i}>0$ and $\lambda_{i j} \in \mathbb{R}$. Here we repeat the proof of proposition 7 given in [68] since it also sets the basis for an efficient FA algorithm.

Proof. Consider the joint covariance matrix of hidden and observed variables,

$$
\left(\begin{array}{l}
X \\
Y
\end{array}\right)=\left(\begin{array}{cc}
\Sigma & \Lambda \\
\Lambda^{t} & \Psi
\end{array}\right)
$$

The entries of this matrix are constrained by the CI statements:

$$
X_{i} \Perp X_{j} \mid\left\{Y_{1}, \ldots, Y_{k}\right\} \quad(1 \leq i<j \leq d),
$$

which translate into the vanishing of the following $(k+1) \times(k+1)$ determinants:

$$
\operatorname{det}\left(\begin{array}{cc}
\sigma_{i j} & \Lambda_{i *} \\
\Lambda_{j *} & \Phi
\end{array}\right)=\operatorname{det}(\Phi)\left(\sigma_{i j}-\Lambda_{i *} \Phi^{-1} \Lambda_{j *}^{t}\right)=0 .
$$

Assuming $i \neq j$ and $\operatorname{det}(\Phi)>0$, equation 5.41 implies that the positive definite Schur complement $\Omega=\Sigma-\Lambda \Phi^{-1} \Lambda^{t}$ is diagonal. By Cholesky decomposition of $\Phi^{-1}$, the covariance matrix $\Sigma=\Omega+\Lambda \Phi^{-1} \Lambda^{t}$ for the observed variables is seen to be in $\mathbf{F}_{\mathbf{d}, \mathbf{k}}$, and all matrices in $\mathbf{F}_{\mathbf{d}, \mathbf{k}}$ can be obtained in this fashion. 


\title{
Chapter 6
}

\section{Graphical Models of Sepsis Incidence and Outcome Prediction in Patients Treated with Statins}

\author{
L'indépendance a toujours été \\ mon désir, la dépendance a \\ toujours été mon destin.
}

Paul Verlaine

\subsection{Introduction}

Statins are a class of drug that lowers cholesterol levels by inhibiting a particular enzyme (3-hidroxi-methylglutaril reductase), which plays a central role in the production of cholesterol in the liver. Increased cholesterol levels have been associated with cardiovascular diseases (CVD), and statins are therefore used in the prevention of these diseases [4]. Apart from its hypolipemic properties, they also exercise anti-inflammatory, immunomodulator and antioxidant actions and are capable of modulating vase reactivity in the coagulation system by means of its actions at endothelial cell level [90, 91]. Recent studies suggest that chronic treatment with statins would present beneficial effects for infection prevention and treatment. There is suggestion as well of a possible beneficial effect in ICU outcome [92, 93, 94, 95, 96, 97, 98, 99, 100, 101]. Despite this evidence, several studies have only found a neutral effect [102], or even a greater mortality in patients treated with statins [103] in this environment. None of the studies reviewed by the author address the effect of statins in patients with severe sepsis or Multiple Organ Dysfunction Syndrome (MODS).

Beginning to fill this gap of knowledge, the current chapter examines the association between the administration of statins in preadmission and the mortality rates in the ICU over a population of 750 patients affected with severe sepsis and MODS by means of algebraic statistical techniques for conditional 
independence analysis and MRFs. It must be noted that the patients' database used for the current study, as described in the following section, is larger than any other used for the same research purposes and comes from one of the biggest hospital ICUs in the Spanish public health care system.

The use of Markov Random Fields and Regression Trees for decision making is a key feature in this context. Clinicians in general might benefit from at least partially automated computer-based decision support, but those clinicians making real-time executive decisions at ICUs in particular will require methods that are not only reliable, but also -and this is a key issue- readily interpretable. Decision trees in general comply nicely with this last requirement, as their predictions can be easily transformed into decision rules amenable to swift implementation at the point-of-care [104].

\subsection{Materials}

The experiments reported in the coming sections are based on a prospective study approved by the Clinical Investigation Ethical Committee of the Vall d'Hebron University Hospital in Barcelona, Spain, which yielded a database collected by the Research Group on Shock, Organic Dysfunction and Resuscitation (SODIR) of Vall d' Hebron's Intensive Care Unit (VH-ICU). The database consisted of data collected of all patients who where admitted in the ICU with severe sepsis and MODS at this hospital between July 2004 and December 2009.

During this period, 750 patients with severe sepsis and MODS were admitted to the ICU (including medical and surgical patients). The mean age of the patients in the analysed database was 57.07 (with standard deviation \pm 16.65 ) years; $47.91 \%$ of patients were female; and the diagnosis on admission was $67.83 \%$ medical and $32.17 \%$ surgical. The origin of primary infection for the cases on the database was $40.28 \%$ pulmonary, $23.20 \%$ abdominal, $10.76 \%$ urinary, $7.21 \%$ skin/muscle, $4.88 \%$ central nervous system (CNS), $1.55 \%$ catheter related, $1.00 \%$ endovascular, $4.99 \%$ biliary, $1.55 \%$ mediastinum, and $4.58 \%$ unknown. Also, $14.13 \%$ of patients $(n=106)$ received preadmission statins.

Organ dysfunction was evaluated by means of the SOFA score [2], which quantifies the dysfunction and failure of six organs/systems (Cardiovascular, Respiratory, CNS, Hepatic, Renal and Haematologic), as shown in Table 6.1, and is scored from 0 (normal function) to 4 points (maximum failure). Severity was evaluated by means of the APACHE II score [1], resulting in a value of $23.03 \pm 9.62$

\subsection{Methods}

\subsubsection{Algebraic Statistical Models}

Algebraic statistics have been successfully applied to problems in the areas of genomics and proteomics, to obtain Maximum Likelihood amino acid sequences in phylogenetics [14]. More generally, algebraic statistics are used in phylogenetics to show the necessary marginal independence conditions in the analysis of biological sequences. The idea behind this approach is that marginal independence conditions induce a Markov Random Field that can be used for inference. 
Table 6.1: List of SOFA scores, with their corresponding mean and standard deviation values.

\begin{tabular}{|c|c|}
\hline Cardiovascular (CV) & $2.86(1.61)$ \\
\hline Respiratory (RESP) & $2.31(1.15)$ \\
\hline Central Nerv. Sys. (CNS) & $0.48(0.99)$ \\
\hline Hepatic (HEPA) & $0.49(0.92)$ \\
\hline Renal (REN) & $1.06(1.20)$ \\
\hline Haematologic (HAEMATO) & $0.78(1.14)$ \\
\hline Global SOFA score & $7.94(3.83)$ \\
\hline
\end{tabular}

A statistical model is defined as a family of distributions over a sample space $\Omega$. In our case, $\Omega$ is finite with cardinal $Q$. If the distributions are given by polynomials over the parameters, this model is defined as an Algebraic Statistical Model. More specifically, let us recall definition 11

Definition 41. Algebraic Statistical Model:

A statistical model that can be specified by means of a variety

$$
\operatorname{Variety}\left(f_{1} \cdots f_{q}, h_{1} \cdots h_{l}\right) \in \mathcal{K}^{d+p+h}
$$

with respect to a set of parameters (with the ideal denoted by IdealVariety) is an Algebraic Statistical Model.

In this case, $X$ is a random variable $X=\left(x_{1}, \ldots, x_{d}\right)$ where each $x_{k}$ takes values in $\{1,2\}$, the model parameters are given by $\Theta=\left(\theta_{1}, \ldots, \theta_{d}\right) ; \psi_{i}(\Theta)$ is defined as $\psi_{i}(\Theta)=P\left(x_{k}=i \mid \Theta\right)$ for some $k \in\{1, \ldots, d\}$ by definition 11 and $\psi$ is restricted to the probability simplex $\left(\Delta^{N-1}\right)$ to guarantee the fulfilment of the Markov condition. More particularly, $\psi$ is defined over a set $U \subseteq \mathbb{R}^{d}$ and $\psi(u) \cap \Delta^{N-1} \subseteq \mathbb{R}^{N}$ (c.f. section 4.4.1).

\subsubsection{Models of Conditional Independence}

In section 4.4.2 we have seen that given three disjoint subsets $A, B, C \neq \varnothing$ of $\left\{X_{1}, \ldots, X_{n}\right\}, A$ is independent of $B$ given $C, A \Perp B \mid C$ if $\operatorname{Prob}(A=a, B=$ $b \mid C)=\operatorname{Prob}(A=a \mid C=c) \operatorname{Prob}(B=b \mid C=c) \forall a, b, c$ such that $\operatorname{Prob}(C=$ c) $>0$. Also the Hammersley-Clifford theorem 10 [105] shows the connection between the parametrization $\psi$ and the collection of conditional independence statements presented below. It is important to note that Definition 23 translates into a set of quadratic equations in the unknowns $\left(p_{i_{1}, \ldots, i_{n}}\right)$.

\subsubsection{Markov Random Fields}

By definition 26 in chapter $4, \mathcal{K}$ is a Markov Random Field (MRF) with respect to graph $\mathcal{G}$ if its joint probability density function factorizes as a product of the individual density functions, conditional on their parent variables. From this definition and theorem 10 we know that the pairwise Markov condition (i.e. each node is independent of its non descendants) will hold. In other words, our support $\left\{X_{1}, \ldots, X_{n}\right\}$ defines a Markov Field. 


\subsubsection{Algebraic Interpolation from Gröbner Bases}

An alternative to the graphical methods presented so far is the application of the algorithm presented in section 4.2.3, which requires the set of unique points (i.e. the design table calculated) or observations matrix. For the sake of clarity let us repeat the most important concepts behind algorithm for calculating the Quotient Basis and its related interpolation polynomial:

- Let $A$ be an $N \times k$ observation matrix with $N$ different support points in $\mathcal{Z}^{k}$. These $N$ distinct points can be represented as the set of solutions of a Gröbner Basis $G(A)$ for a given term ordering $\tau$ (c.f. definition 4.1).

- For the term ordering $\tau$ and ideal $I$ any polynomial can be written as

$$
p(x)=\sum_{j=1}^{N} I_{j}(A) g_{j}(A)+r(A) .
$$

where $\mathrm{r}(\mathrm{A})$ is unique.

- The monomials of $r(A)$ from a subset $\operatorname{EST}_{\tau}$ (Quotient Basis), which comprises all monomials that are not divisible by the leading terms of $G$ for the given term ordering $\tau$.

The algorithm that we propose to calculate the interpolation polynomial is (c.f. section 4.2.3):

1. Input: matrix with unique points $A$ and relative frequencies $q$.

2. Define a term ordering $\tau$ (for example lexicographic).

3. Calculate the ideal of matrix A (in our case, this is done with ApCoCoA [61]).

4. Calculate the reduced Gröbner Basis $G$ (this can be also calculated with the function IdealOfPoints [62] in ApCoCoA).

5. Identify the subset $\operatorname{EST}_{\tau}$ (i.e. identify the sub-set of monomials not divided by $G$ ).

6. Let $L$ be the logarithm of the monomials of $\operatorname{EST}_{\tau}$ (i.e. exponents). Write $\mathrm{EST}_{\tau}=\left\{a^{\alpha}\right\}_{\alpha \in L}$.

7. Write the polynomial interpolator as: $p(a)=\sum_{\alpha \in L} \eta_{\alpha} a^{\alpha}$.

8. Substitute the values of $a$ in $p\left(a_{k}\right)=q_{k} k \in\{1, \ldots, N\}$ and solve the polynomial system for the parameters $\eta_{\alpha}$. The solution is guaranteed and unique by the construction of $G$. 


\subsection{Results}

\subsubsection{Study of the Incidence of Sepsis with Bayes Net- works over the basal SOFA Score}

As it has been stated in 2.4.1, the basal SOFA Score is the result of adding the dysfunction score for 6 different organ systems. More particularly, a SOFA score greater than 1 is demonstrative of MODS while a Cardiovascular SOFA greater than 2 is related to Septic Shock. By the very definition of the score, it becomes apparent that Severe Sepsis, Shock, MODS and the ICU result are dependent on each other and that the SOFA score is related to Severe Sepsis $(\mathrm{SOFA}=1)$, to Shock (SOFA CV $>2)$ and MODS $(\mathrm{SOFA}>1)$. In the light of what has been described in this section, this will correspond to a Bayes Network with a corresponding grid depicted as follows:

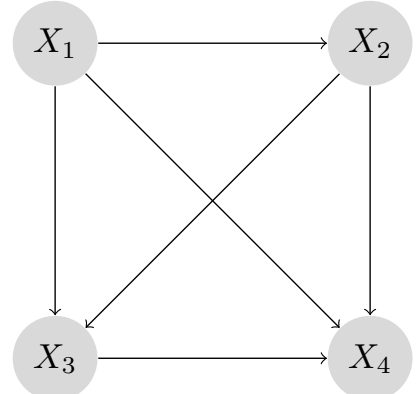

More particularly, node 1 is the unobserved number of Severe Sepsis Patients. This is due to the fact that some patients with Severe Sepsis are not admitted in the ICU because their severity is not very important. In this MRF, node 2 corresponds to Septic Shock, node 3 corresponds to MODS and Node 4 to the ICU result. This Bayes Network implemented with the Bayes Net Toolbox ${ }^{1}$ yielded an incidence of Severe Sepsis for Hospital Universitari Vall d'Hebron of 187.22 cases/year (i.e. 41.61 cases/100,000 habitants) out of which 164 cases enter this ICU every year (this is the annual incidence of patients that we have in our dataset). This incidence is not very different from that in other regions of Spain, such as Madrid (141 cases /100,000 habitants), or Castilla y León (250 cases / 100,000 habitants) ${ }^{2}$.

\subsubsection{Marginal Dependence Between Preadmission Use of Statins and the ICU Outcome}

We aim to find the relation between the administration of statin drugs prior to ICU admission and the mortality rate in severe sepsis patients. For that, we tested the null hypothesis that the ICU outcome is independent of the preadmission use of statins for given APACHE II and SOFA scores. More specifically by proposition 4.4 .2 from chapter 4 , we tested the following hypothesis $H_{o}$ :

$$
H_{o}:\left\{X_{1}\right\} \Perp\left\{X_{4}\right\} \mid\left\{X_{2}\right\},\left\{X_{3}\right\} .
$$

\footnotetext{
${ }^{1}$ available online in http://code.google.com/p/bnt/

${ }^{2}$ personal communication with Juan Carlos Ruiz from the ICU at Hospital Universitari Vall d'Hebron, Barcelona
} 
where $\left\{X_{1}\right\}$ is the ICU outcome, $\left\{X_{4}\right\}$ the preadmission use of statins, $\left\{X_{3}\right\}$ the SOFA score, and $\left\{X_{2}\right\}$ the APACHE II score. In our case, $\left\{X_{1}\right\}$ is 1 for ICU survival and 0 corresponds to exitus. Also, $\left\{X_{4}\right\}$ is 1 if the patient followed preadmission statin treatment, and 0 if the patient did not follow it. The APACHE II and SOFA scores were stratified according to the minimum value that results in a significant increase in the mortality rates (see Figs. 6.1 and 6.2). This means that APACHE II scores lower than or equal to 21 were set to 0 , while they were set to 1 if the APACHE II was above this threshold. With a similar criterion, SOFA scores lower than or equal to 7 were set to 0 , while they were set to 1 if the SOFA score was above the selected threshold.

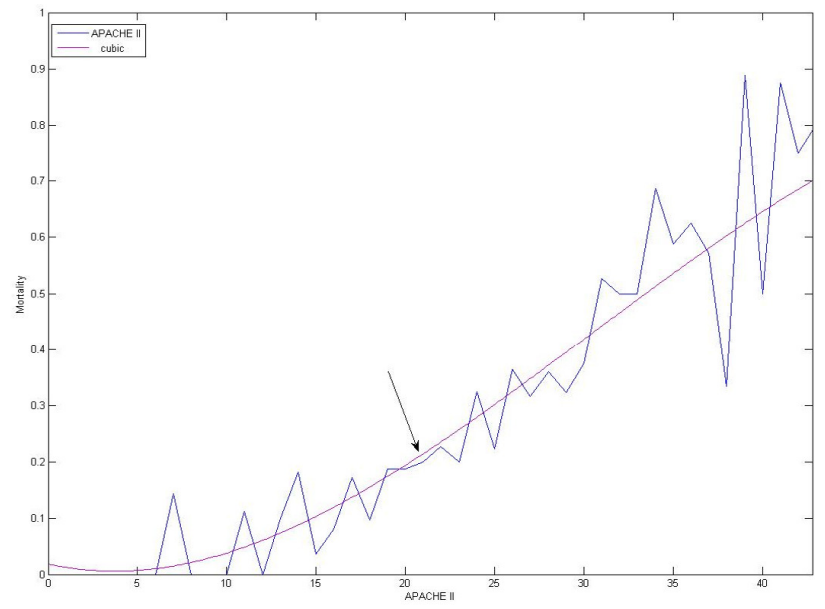

Figure 6.1: APACHE II threshold selection: The blue curve represents the true APACHE II mortality rate, whilst the smooth red curve is the APACHE II mortality rate interpolated with a cubic polynomial. The arrow points to the first inflection point of the polynomial, which, in this study, corresponds to the selected APACHE II threshold for stratification (i.e. APACHE II $=21$ ). This means that APACHE II scores lower than this threshold are set to 2 in our MRF. Conversely, the APACHE II values higher than 21 are set to 1 in our MRF. This threshold is consistent with standard clinical practice [1]

From section 6.3 .1 , we now have a $4 \times 2 \times 2$ matrix $M$ of relative frequencies and from definition 2 we know that all the minors of $M$ should have a rank lower than 1 for $H_{0}$ to hold. In our case the four minors of $M$ are: 


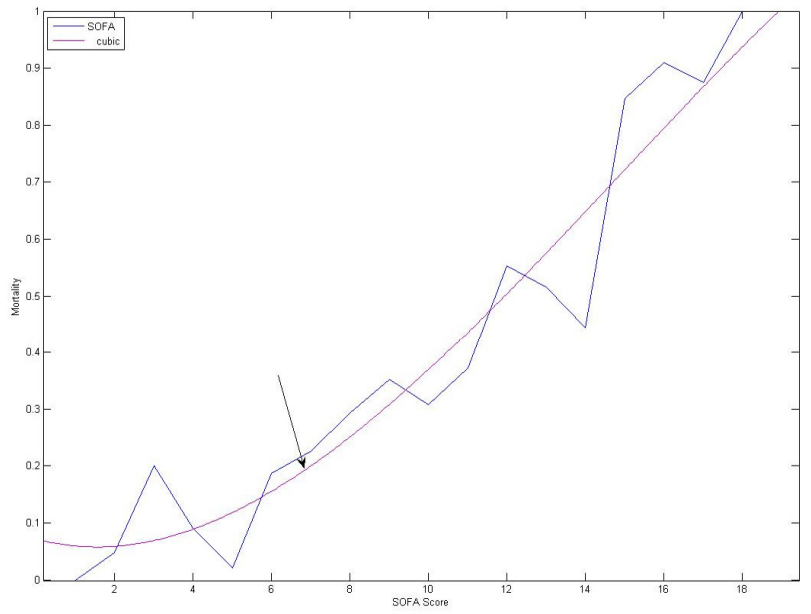

Figure 6.2: SOFA Score threshold selection: The blue curve represents the true SOFA SCORE mortality rate, whilst the smooth red curve is the SOFA Score mortality rate interpolated with a cubic polynomial. As in the previous figure, the arrow points to the first inflection point of the polynomial, which is selected as SOFA Score threshold for stratification (i.e. SOFA =7). This means that SOFA scores lower than this threshold are set to 2 in our MRF. Conversely, the SOFA values higher than 7 are set to 1 in our MRF. This threshold is consistent with standard clinical practice.

$$
\begin{aligned}
& M_{0,0}=\left(\begin{array}{ll}
0.0217 & 0.0014 \\
0.1655 & 0.0176
\end{array}\right) \\
& M_{0,1}=\left(\begin{array}{ll}
0.0163 & 0.0014 \\
0.0380 & 0.0054
\end{array}\right) \\
& M_{1,0}=\left(\begin{array}{ll}
0.0258 & 0.0027 \\
0.1723 & 0.0285
\end{array}\right) \\
& M_{1,1}=\left(\begin{array}{ll}
0.1981 & 0.0285 \\
0.2266 & 0.0502
\end{array}\right)
\end{aligned}
$$

The rank of all the minors above was calculated using the singular value decomposition (SVD) algorithm [106]. Table 6.2 shows that all minors of matrix $M$ are full rank, and, therefore, the null hypothesis can be rejected. This means that the ICU outcome is in fact marginally dependent on the preadmission use of statins for given APACHE II and SOFA scores (severity and organ dysfunction). These results are also consistent with a $\chi^{2}$ test, which rejected the null hypothesis with a $p=0$. However, this $\chi^{2}$ is only giving us the dependence between ICU outcome and preadmission use of Statins without giving any 'contextual' information about this outcome related to organ dysfunction (SOFA 
Table 6.2: Ranks of Minors Obtained with SVD

\begin{tabular}{|c|c|c|}
\hline Minor & Rank & Tolerance \\
\hline$M_{0,0}$ & 2 & $5.55 \cdot 10^{-17}$ \\
\hline$M_{0,1}$ & 2 & $1.39 \cdot 10^{-17}$ \\
\hline$M_{1,0}$ & 2 & $5.55 \cdot 10^{-17}$ \\
\hline$M_{1,1}$ & 2 & $1.11 \cdot 10^{-16}$ \\
\hline
\end{tabular}

score) and severity (APACHE II).

In order to construct the graph $\mathcal{G}$ we also need to study the marginal dependences between the rest of variables. The required marginal dependence tables for all variables are presented in tables 6.3 to 6.7 .

For $H_{0}: X_{1} \Perp X_{2} \mid X_{3}, X_{4}$ :

$$
\begin{gathered}
M_{0,0}=\left(\begin{array}{ll}
0.0217 & 0.0258 \\
0.1655 & 0.1723
\end{array}\right) \\
M_{0,1}=\left(\begin{array}{ll}
0.00140 & 0.00270 \\
0.01760 & 0.02850
\end{array}\right) \\
M_{1,0}=\left(\begin{array}{ll}
0.0163 & 0.1981 \\
0.0380 & 0.2266
\end{array}\right) \\
M_{1,1}=\left(\begin{array}{ll}
0.00140 & 0.02850 \\
0.00540 & 0.05020
\end{array}\right)
\end{gathered}
$$

Table 6.3: Ranks, $H_{0}:\left\{X_{1}\right\} \Perp\left\{X_{2}\right\} \mid\left\{X_{3}\right\},\left\{X_{4}\right\}$

\begin{tabular}{|c|c|c|}
\hline Minor & Rank & Tolerance \\
\hline$M_{0,0}$ & 2 & $1.07 \cdot 10^{-16}$ \\
\hline$M_{0,1}$ & 2 & $1.49 \cdot 10^{-17}$ \\
\hline$M_{1,0}$ & 2 & $1.35 \cdot 10^{-16}$ \\
\hline$M_{1,1}$ & 2 & $2.57 \cdot 10^{-17}$ \\
\hline
\end{tabular}

For $H_{0}:\left\{X_{1}\right\} \Perp\left\{X_{3}\right\} \mid\left\{X_{2}\right\},\left\{X_{4}\right\}$ :

$$
\begin{gathered}
M_{0,0}=\left(\begin{array}{ll}
0.0217 & 0.0163 \\
0.1655 & 0.0380
\end{array}\right) \\
M_{0,1}=\left(\begin{array}{ll}
0.00140 & 0.00140 \\
0.01760 & 0.00540
\end{array}\right) \\
M_{1,0}=\left(\begin{array}{ll}
0.0258 & 0.1981 \\
0.1723 & 0.2266
\end{array}\right) \\
M_{1,1}=\left(\begin{array}{ll}
0.00270 & 0.02850 \\
0.02850 & 0.05020
\end{array}\right)
\end{gathered}
$$


Table 6.4: Ranks, $H_{0}:\left\{X_{1}\right\} \Perp\left\{X_{3}\right\} \mid\left\{X_{2}\right\},\left\{X_{4}\right\}$

\begin{tabular}{|c|c|c|}
\hline Minor & Rank & Tolerance \\
\hline$M_{0,0}$ & 2 & $7.62 \cdot 10^{-17}$ \\
\hline$M_{0,1}$ & 2 & $8.21 \cdot 10^{-18}$ \\
\hline$M_{1,0}$ & 2 & $1.50 \cdot 10^{-16}$ \\
\hline$M_{1,1}$ & 2 & $2.82 \cdot 10^{-17}$ \\
\hline
\end{tabular}

For $H_{0}: X_{2} \Perp X_{3} \mid X_{1}, X_{4}$ :

$$
\begin{aligned}
& M_{0,0}=\left(\begin{array}{ll}
0.0217 & 0.0163 \\
0.0258 & 0.1981
\end{array}\right) \\
& M_{0,1}=\left(\begin{array}{ll}
0.0014 & 0.0014 \\
0.0027 & 0.0285
\end{array}\right) \\
& M_{1,0}=\left(\begin{array}{ll}
0.1655 & 0.0380 \\
0.1723 & 0.2266
\end{array}\right) \\
& M_{1,1}=\left(\begin{array}{ll}
0.0176 & 0.0054 \\
0.0285 & 0.0502
\end{array}\right)
\end{aligned}
$$

Table 6.5: Ranks, $H_{0}:\left\{X_{2}\right\} \Perp\left\{X_{3}\right\} \mid\left\{X_{1}\right\},\left\{X_{4}\right\}$

\begin{tabular}{|c|c|c|}
\hline Minor & Rank & Tolerance \\
\hline$M_{0,0}$ & 2 & $8.91 \cdot 10^{-17}$ \\
\hline$M_{0,1}$ & 2 & $1.27 \cdot 10^{-17}$ \\
\hline$M_{1,0}$ & 2 & $1.41 \cdot 10^{-16}$ \\
\hline$M_{1,1}$ & 2 & $2.63 \cdot 10^{-17}$ \\
\hline
\end{tabular}

For $H_{0}: X_{2} \Perp X_{4} \mid X_{1}, X_{3}$ :

$$
\begin{aligned}
& M_{0,0}=\left(\begin{array}{ll}
0.0217 & 0.0014 \\
0.0258 & 0.0027
\end{array}\right) \\
& M_{0,1}=\left(\begin{array}{ll}
0.0163 & 0.0014 \\
0.1981 & 0.0285
\end{array}\right) \\
& M_{1,0}=\left(\begin{array}{ll}
0.1655 & 0.0176 \\
0.1723 & 0.0285
\end{array}\right) \\
& M_{1,1}=\left(\begin{array}{ll}
0.0380 & 0.0054 \\
0.2266 & 0.0502
\end{array}\right)
\end{aligned}
$$


Table 6.6: Ranks, $H_{0}:\left\{X_{2}\right\} \Perp\left\{X_{4}\right\} \mid\left\{X_{1}\right\},\left\{X_{3}\right\}$

\begin{tabular}{|c|c|c|}
\hline Minor & Rank & Tolerance \\
\hline$M_{0,0}$ & 2 & $1.50 \cdot 10^{-17}$ \\
\hline$M_{0,1}$ & 2 & $8.92 \cdot 10^{-17}$ \\
\hline$M_{1,0}$ & 2 & $1.07 \cdot 10^{-16}$ \\
\hline$M_{1,1}$ & 2 & $1.04 \cdot 10^{-16}$ \\
\hline
\end{tabular}

For $H_{0}: X_{3} \Perp X_{4} \mid X_{1}, X_{2}$ :

$$
\begin{aligned}
& M_{0,0}=\left(\begin{array}{ll}
0.0217 & 0.0014 \\
0.0163 & 0.0014
\end{array}\right) \\
& M_{0,1}=\left(\begin{array}{ll}
0.0258 & 0.0027 \\
0.1981 & 0.0285
\end{array}\right) \\
& M_{1,0}=\left(\begin{array}{ll}
0.1655 & 0.0176 \\
0.0380 & 0.0054
\end{array}\right) \\
& M_{1,1}=\left(\begin{array}{ll}
0.1723 & 0.0285 \\
0.2266 & 0.0502
\end{array}\right)
\end{aligned}
$$

Table 6.7: Ranks, $H_{0}:\left\{X_{3}\right\} \Perp\left\{X_{4}\right\} \mid\left\{X_{1}\right\},\left\{X_{2}\right\}$

\begin{tabular}{|c|c|c|}
\hline Minor & Rank & Tolerance \\
\hline$M_{0,0}$ & 2 & $1.21 \cdot 10^{-17}$ \\
\hline$M_{0,1}$ & 2 & $8.96 \cdot 10^{-17}$ \\
\hline$M_{1,0}$ & 2 & $7.58 \cdot 10^{-17}$ \\
\hline$M_{1,1}$ & 2 & $1.29 \cdot 10^{-16}$ \\
\hline
\end{tabular}

\subsubsection{Study of the Protective Effect of Preadmission Use} of Statins with MRFs

The graph $\mathcal{G}$ resulting from the calculations in section 6.4 .2 is the fully connected graph:

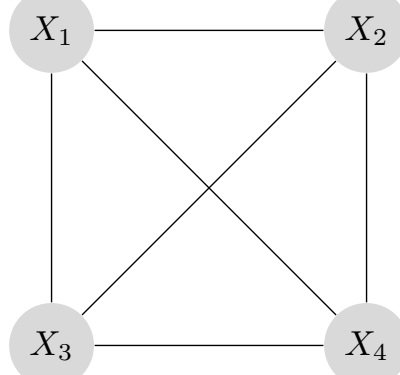


Table 6.8: Marginal Probabilities for ICU results

\begin{tabular}{|c|c|c|c|c|}
\hline Statins & SOFA & APACHE II & Result $=1$ & Result $=2$ \\
\hline 1 & 1 & 1 & 0.64 & 0.36 \\
\hline 2 & 1 & 1 & 0.53 & 0.47 \\
\hline 1 & 2 & 1 & 0.80 & 0.20 \\
\hline 2 & 2 & 1 & 0.70 & 0.30 \\
\hline 1 & 1 & 2 & 0.91 & 0.09 \\
\hline 2 & 1 & 2 & 0.87 & 0.13 \\
\hline 1 & 2 & 2 & 0.93 & 0.07 \\
\hline 2 & 2 & 2 & 0.88 & 0.12 \\
\hline
\end{tabular}

The marginal probabilities for the ICU result node $X_{1}$ are summarized in table 6.8. In this table, the preadmission use of Statins, Moderate/Low SOFA scores and Moderate/Low APACHE II scores are coded as 2. Also ICU result has been coded as 1 for survival and 2 for exitus.

From table 6.8 it becomes apparent that preadmission use of Statins play an important role for ICU outcome. This effect becomes more apparent for high severity and moderate organ dysfunction as measured by the SOFA score and APACHE II (0.80 vs 0.70). However, this effect is more important for both high organ dysfunctions and severities (0.64 vs 0.53$)$.

\subsubsection{Study of Interactions by means of Algebraic Inter- polation}

Since we have already studied the dependence between the different factors, we would like to study this same relation algebraically and also provide an interpolator for new points (i.e. provide the algebraic equivalent of our table). The methodology proposed uses the Algebraic Interpolation Method as presented in chapter 4 . This methodology is best suited for bigger tables or multi-dimensional matrices (tensors).

The input matrix for the algorithm is the table 6.8. The vanishing ideal for this table and the lexicographic ordering $\tau$ calculated with ApCoCoA [61] is

$$
I=\left\langle x_{3}^{2}-3 x_{3}+2, x_{2}^{2}-3 x_{2}+2, x_{1}^{2}-3 x_{1}+2\right\rangle .
$$

The Gröbner Basis corresponding to this Ideal and ordering $\tau$ is

$$
G=\left\{x_{3}^{2}-3 x_{3}+2, x_{2}^{2}-3 x_{2}+2, x_{1}^{2}-3 x_{1}+2\right\}
$$

The corresponding Quotient Basis is

$$
B=\left\{1, x_{3}, x_{2}, x_{2} x_{3}, x_{1}, x_{1} x_{3}, x_{1} x_{2}, x_{1} x_{2} x_{3}\right\} .
$$

Our Interpolation Polynomial has the form: 


$$
\begin{aligned}
P\left(x_{1}, x_{2}, x_{3}\right)=\eta_{7} x_{1} x_{2} x_{3}+\eta_{6} x_{1} x_{2} & +\eta_{5} x_{1} x_{3} \\
& -\eta_{4} x_{2} x_{3}-\eta_{3} x_{1}+\eta_{2} x_{2}+\eta_{1} x_{3}+\eta_{0}
\end{aligned}
$$

Solving for $\left\{x_{1}, x_{2}, x_{3}\right\}$ by substitution and also knowing that $\eta_{0}=1-$ $\sum_{i=1}^{7} \eta_{i}$ yields the interpolation polynomial

$$
\begin{aligned}
P\left(x_{1}, x_{2}, x_{3}\right)= & -1 / 50 x_{1} x_{2} x_{3}+3 / 100 x_{1} x_{2}+9 / 100 x_{1} x_{3} \\
& -3 / 25 x_{2} x_{3}-21 / 100 x_{1}+27 / 100 x_{2}+8 / 25 x_{3}+7 / 25
\end{aligned}
$$

The leading term of this polynomial $-1 / 50 x_{1} x_{2} x_{3}$ also shows the relation between the preadmission use of statins, severity (APACHE II) and organ dysfunction (SOFA score). Of course, the dependencies in our polynomial are equivalent to those presented in the former section.

\subsubsection{Study of the Protective Effect of Preadmission Use of Statins with Regression Trees}

Once established the marginal dependence between preadmission treatment with statins and the ICU outcome, such dependence was analysed in further detail using regression trees, following the method described in section 5.1 [81, 82]. That is, a regression tree was implemented to study the probability of ICU survival (i.e. $x_{i}$ includes the stratified SOFA Score, the APACHE II Score and the preadmission use of statins, whereas $y_{i}$ is the ICU outcome, with $\left.y_{i} \in\{0,1\}\right)$.

Fig. 6.3 displays the resulting regression tree. First of all, it shows that the most significant parameter is the APACHE II score, which measures the severity of the illness, as it generates the first branching of the tree from the root node. Each of the branches is now commented separately:

- Branch APACHE II<0.5 (Moderate/Low Severity):

- For moderate/low Organ Dysfunction (i.e. SOFA $<0.5)$ the patients that received statins $(\mathrm{EST} \geq 0.5)$ present a survival rate of $92.0 \%$ $(\mathrm{n}=231)$, whilst those who did not $(\mathrm{EST}<0.5)$ present a survival rate of $90.8 \%(n=25)$. This result suggests that for moderate/low Organ Dysfunction and moderate/low Severity measured with the APACHE II score, the preadmission use of statins has almost no effect on ICU outcome.

- For higher Organ Dysfunction (i.e. SOFA > 0.5), the patients that received preadmission statins present a survival rate of $92.3 \%(n=13)$ : far higher than those who did not, which present a survival rate of $74.5 \%(\mathrm{n}=55)$.

- Branch APACHE II>0.5 (High Severity):

- For moderate/low Organ Dysfunction $(\mathrm{SOFA}<0.5)$, patients that were not treated with statins prior to the admission in the ICU present a higher survival rate than those that received treatment 
$(73.2 \%(\mathrm{n}=84)$ vs. $69.2 \%(\mathrm{n}=13))$. This result suggests that under these circumstances, the preadmission use of statins may play a negative role in ICU outcome. It is however important to note that this branch corresponds to patients who were severely ill in spite of their Severe Sepsis (for example a patient with terminal cancer that got infected during the course of their illness). Therefore, the mortality rates here are more related to underlying comorbidities rather than Severe Sepsis.

- For higher Organ Dysfunction or Severe MODS, the protective effect of preadmission use of statins becomes more apparent $(62.7 \%$ $(\mathrm{n}=55)$ vs. $50.0 \%(\mathrm{n}=274)$ survival rate $)$. This is an important result that suggests that statins play a protective role against Severe Organ Dysfunction.

\subsubsection{Study of Septic Shock Incidence with Regression Trees}

Patients with a high APACHE II and high SOFA scores $>7$ (i.e. SOFA $\geq 0.5$ in the tree) very often suffer Septic Shock. In our database, $94.23 \%$ of patients with a SOFA score greater than 7 also suffered Septic Shock.

The probability of Shock for the population under study (patients who where admitted in the ICU, with and without preadmission use of statins) was also investigated by means of a regression tree with exactly the same inputs as those used in the mortality prediction study.

As revealed by the resulting tree, displayed in Fig. 6.4, the most predictive variable in this case turns out to be the SOFA score. This is due to the fact that the SOFA score also measures the cardiovascular function and those patients with a Cardiovascular SOFA greater than 2 are always administered vasoactive drugs (normally Noradrenaline/Norepinephrine) at different perfusion rates, resulting in different scores. These perfusion rates depend on the severity of the Septic Shock. Again, these first two branches are discussed separately:

- Branch SOFA <0.5 (Moderate/Low Organ Dysfunction):

- For moderate/low Severity (i.e. APACHE II < 0.5) the patients that received statins present a similar but higher Shock rate than those who did not (i.e. $48.00 \%(\mathrm{n}=25)$ vs $44.10 \%(\mathrm{n}=231))$.

- For higher Severity (i.e. APACHE II > 0.5), exactly the same effect was found. Patients that received preadmission statins present a Shock rate of $69.23 \%(n=13)$, while those who did not receive them present a Shock rate of $65.85 \%(n=84)$.

- Branch SOFA>0.5 (High Organ Dysfunction):

- For moderate/low Severity (APACHE II < 0.5), all patients that received preadmission statins suffered a Septic Shock $(n=55)$. On the other hand, patients that did not receive statins present a Shock rate of $92.72 \%(\mathrm{n}=274)$.

- For higher Severity, the results for both populations are quite similar. More specifically, the patients who received statins present a Shock rate of $98.04 \%(n=13)$ and those who did not $97.08 \%(n=55)$. 


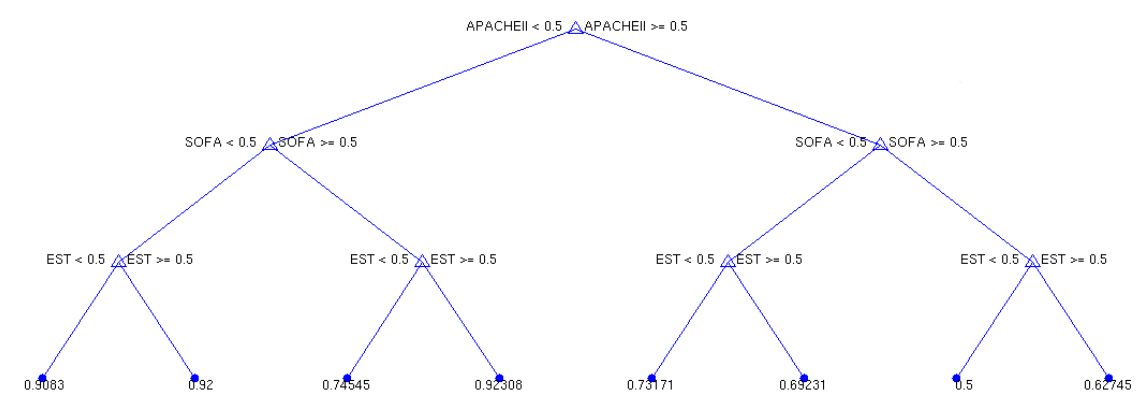

Figure 6.3: Regression Tree for Probability of Survival.

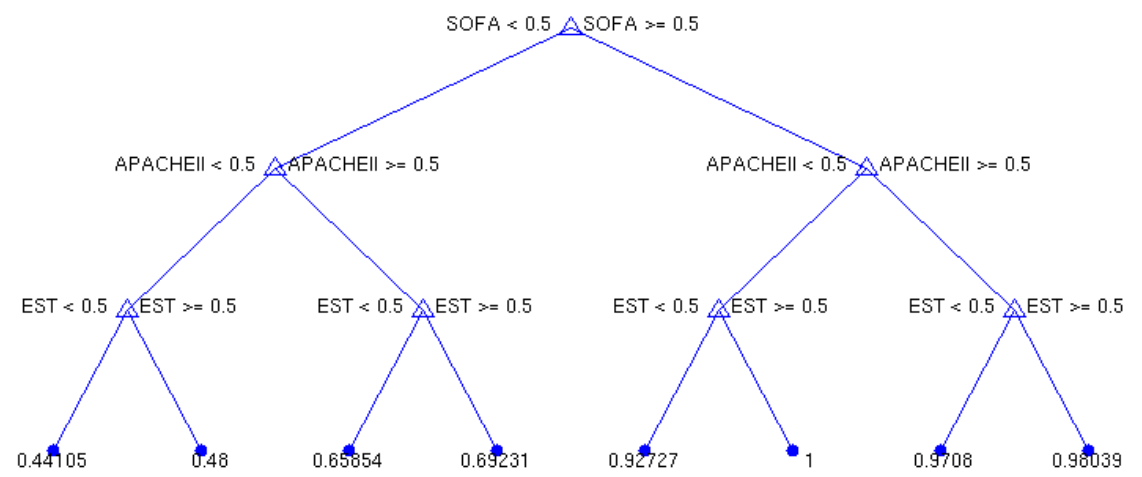

Figure 6.4: Regression Tree for Shock Prediction

From the tree in Fig. 6.4, it becomes apparent that the Shock rates for both populations are quite similar. However, it is also important to note that the Septic Shock rates for the population that received preadmission statins are slightly higher than for those who did not. One possible explanation for this result is that the former population present higher comorbidities than the latter one. In other words, some patients who were administered statins in preadmission were admitted in the ICU for a different base pathology than Sepsis, and only developed Sepsis while at the ICU. This fact has not been taken into account in this study, whose main objective is to study the role of preadmission statins in ICU mortality. In any case, it could tentatively be concluded that the use of statins at preadmission does not provide significant protection against Septic Shock if comorbidities are not taken into account.

\subsection{Conclusion}

There is clinical evidence that the use of statins plays an important role in the prognosis of severe sepsis. Despite this, the studies that have addressed this problem in the critical care field have so far been inconclusive.

We have provided sound evidence that the administration of statin drugs 
plays an important role in the prognosis of severe sepsis in the ICU context. A simple method to evaluate the dependence between the preadmission use of statins and the ICU outcomes by means of ASMs have been presented. These methods (Marginal Dependence Analysis and Polynomial Interpolation) have revealed a clear dependence between the statins treatment in preadmission and the ICU outcome for given severity and organ dysfunction/shock levels, respectively measured with the APACHE II and the SOFA scores in severe sepsis patients.

The protective effect of statins has been further studied using MRFs and Regression Trees. The main conclusion of this study is that these protective effects become more important for severe multi-organic failures accompanied by high APACHE II scores (showing a decrease in the mortality rate of about 10\%). This same effect is also observed in moderate organ dysfunction syndromes and high severities.

This is an encouraging result that is consistent with clinical practice. MRFs provide transparent rules that could be straightforwardly been used in ICU practice.

The effect of statins on the prediction of septic shock occurrence has also been studied. Our first results indicate that the preadmission use of statins does not present a significant protection against septic shock if comorbidities are not taken into account. The inclusion of comorbidities in this research should be the matter of future investigation.

The relevance of the obtained results is enhanced by the fact that the severe sepsis patients' database used for the current study is, to the best of our knowledge, the largest one used to address this problem and comes from one of the biggest hospital ICU in the Spanish public health care system. 


\section{Chapter 7}

\section{Severe Sepsis Mortality Prediction Using an Interpretable Latent Data Representation}

No renuncio a nada, simplemente hago lo que puedo para que las cosas me renuncien a mi.

Julio Cortázar

\subsection{Introduction}

In this chapter, we propose the use of a latent model-based feature extraction approach to obtain new sets of descriptors, or prognostic factors, for the prediction of mortality due to Sepsis. The reported experimental results are readily interpretable. Interpretation is, needless to say, a sensitive issue in the medical ambit, and one that should not be underestimated: the lack of translation of the prognostic factors into usable clinical knowledge would risk rendering the proposed approach useless [107].

In the reported experiments, the obtained prognostic factors are used to predict mortality through standard logistic regression (LR), a method commonly used in medical applications $[108,109]$ and widely trusted by clinicians. The prediction accuracy results herein reported improve on those obtained with current standard data descriptors and therefore provide support for the use of these new factors as risk-of-death predictors in ICU environments.

\subsection{Materials}

As in previous chapters, this work resorts to a prospective observational cohort study of adult patients with severe sepsis. The study was conducted at the 
Critical Care Department of the Vall d' Hebron University Hospital (Barcelona, Spain), and it was approved by the Research Ethics Committee of the Hospital. The database consists of data from patients with severe sepsis, collected at the ICU by the Research Group in Shock, Organic Dysfunction and Resuscitation (SODIR), between June, 2007 and December, 2010. During this period, 354 patients with severe sepsis (medical and surgical patients) were admitted in the ICU.

The mean age of the patients in the database was 57.08 (with standard deviation \pm 16.65 ) years; $40 \%$ of patients were female and the diagnosis on admission was $56.15 \%$ medical and $44.85 \%$ surgical. The origin of primary infection for the cases on the database was $40.24 \%$ pulmonary, $23.17 \%$ abdominal, $10.75 \%$ urinary, $7.21 \%$ skin/muscle, $4.88 \%$ central nervous system (CNS), $1.55 \%$ catheter related, $1.00 \%$ endovascular, $2.22 \%$ biliary, $4.99 \%$ mediastinum and $3.99 \%$ unknown. The mortality rate for this extended dataset was $26.32 \%$.

The collected data show the worst values for all variables during the first 24 hours of evolution for Severe Sepsis. Organ dysfunction was evaluated through the SOFA score system [2], which objectively measures organ dysfunction for 6 organs/systems, the details of which are provided in Table 7.1. Severity was evaluated by means of the APACHE II score (for further reference, see [1]). The APACHE II score was $23.03 \pm 9.62$ for the population under study.

Table 7.1: List of SOFA scores, with their corresponding mean and standard deviation values for the population under study (scoring organ dysfunction).

\begin{tabular}{|c|c|}
\hline Cardiovascular (CV) & $2.86(1.62)$ \\
\hline Respiratory (RESP) & $2.31(1.15)$ \\
\hline Central Nerv. Sys. (CNS) & $0.48(1.00)$ \\
\hline Hepatic (HEPA) & $0.48(0.92)$ \\
\hline Renal (REN) & $1.06(1.20)$ \\
\hline Haematologic (HAEMATO) & $0.78(1.14)$ \\
\hline Global SOFA score & $7.94(3.86)$ \\
\hline Dysf. Organs (SOFA 1-2) & $1.68(1.09)$ \\
\hline Failure Organs (SOFA 3-4) & $1.51(1.02)$ \\
\hline Total Dysf. Organs & $3.18(1.32)$ \\
\hline
\end{tabular}

The specific set of 34 features used for the mortality prediction analyses in this chapter are listed in Table 7.2. Input data was scaled to have zero mean and a standard deviation of 1 .

\subsection{Results}

\subsubsection{Diagnosis of the Factor Analysis Model}

Given that the variables of the model do not follow a Gaussian distribution, we proceeded to test if $\Omega$ is loaded in its diagonal. After computation of the covariance of the residual error matrix, we calculate the sum of the diagonal 
Table 7.2: List of variables used in this study.

\begin{tabular}{|c|c|}
\hline Variable & Description \\
\hline v1 & Age \\
\hline v2 & Gender \\
\hline v3 & Sepsis Focus \\
\hline $\mathrm{v} 4$ & Germ Class \\
\hline v5 & Polimicrobial Infection \\
\hline v6 & Base Pathology \\
\hline v7 & Cardiovascular SOFA score \\
\hline v8 & Respiratory SOFA score \\
\hline v9 & CNS SOFA score \\
\hline v10 & Hepatic SOFA Score \\
\hline v11 & Renal SOFA Score \\
\hline v12 & haematologic SOFA Score \\
\hline v13 & Total SOFA Score \\
\hline v14 & Dysfunctional Organs for SOFA $1-2$ \\
\hline v15 & Dysfunctional Organs for SOFA 3-4 \\
\hline v16 & Total Number of Dysfunctional Organs \\
\hline v17 & Mechanical Ventilation \\
\hline v18 & Oxygenation Index $\mathrm{PaO}_{2} / \mathrm{FiO}_{2}$ \\
\hline v19 & Vasoactive Drugs \\
\hline $\mathrm{v} 20$ & Platelet Count \\
\hline $\mathrm{v} 21$ & APACHE II Score \\
\hline $\mathrm{v} 22$ & Surviving Sepsis Campaign Bundles 6h \\
\hline $\mathrm{v} 23$ & Haemocultures $6 \mathrm{~h}$ \\
\hline v24 & Antibiotics $6 \mathrm{~h}$ \\
\hline $\mathrm{v} 25$ & Volume $6 \mathrm{~h}$ \\
\hline $\mathrm{v} 26$ & $\mathrm{O}_{2}$ Central Venous Saturation $6 \mathrm{~h}$ \\
\hline $\mathrm{v} 27$ & Haematocrit $6 \mathrm{~h}$ \\
\hline $\mathrm{v} 28$ & Transfusions $6 \mathrm{~h}$ \\
\hline v29 & Dobutamine $6 \mathrm{~h}$ \\
\hline v30 & Surviving Sepsis Campaign Bundles 24h \\
\hline v31 & Glycaemia $24 \mathrm{~h}$ \\
\hline v32 & PPlateau \\
\hline v33 & Worst Lactate \\
\hline v34 & $\mathrm{O}_{2}$ Central Venous Saturation \\
\hline
\end{tabular}


elements was compared to the off-diagonal ones, for $i \in\{1 \ldots d\}$. Specifically, the value of $K$ so that

$$
\left|\omega_{i i}\right| \geq K \sum_{j=1, j \neq i}^{d}\left|\omega_{i j}\right|
$$

was calculated, turning out to be $K=17.46$ for all $\omega_{i i}$. Because the maximum off-diagonal element is much lower than any of the diagonal elements, diagonal dominance is clear and it can be assumed that all possible interactions between variables are accounted by the matrix $\Lambda$ with 14 factors. The number of factors have been selected according to the likelihood ratio presented in [110], which proposes to select the minimum number of factors that assymptotically give a $\chi^{2}$ distribution.

\subsubsection{Factor Interpretation from a Clinical Viewpoint}

As described in the previous subsection 7.3.1, the application of FA resulted in a consistent 14-factor model of the original data set. The cumulative proportion of total (standardized) sample variance explained by this model was found to be $83.27 \%$.

Table 7.3 summarizes the matrix of loadings corresponding to the original variables listed in Table 7.2. Taking into consideration the highest factor loadings (in absolute value) for every given variable, these factors were mapped into different easily interpretable clinical descriptors, explained as follows:

- Factor 1: Related to cardiovascular function and, more specifically, to the cardiovascular SOFA score and vasoactive drugs c.f. table 6.1.

- Factor 2: Corresponds to haematologic function (haematologic SOFA score and platelet count).

- Factor 3: Corresponds to respiratory function, Respiratory SOFA score and $\mathrm{PaO}_{2} / \mathrm{FiO}_{2}$ ratio.

- Factor 4: Corresponds to the use of mechanical ventilation and PPlateau.

- Factor 5: Corresponds to the 24h SSC bundles and glycaemic indices.

- Factor 6: Related to the micro-organism producing the Sepsis and whether this sepsis polimicrobial or not.

- Factor 7: Corresponds to renal function measured by the SOFA score and total SOFA score.

- Factor 8: Corresponds to the administration of antibiotics and haemocultures taken during the first $6 \mathrm{~h}$ of ICU stay.

- Factor 9: Relates to the number of organs in dysfunction for a moderate SOFA and the total number of organs in dysfunction.

- Factor 10: Related to the hepatic function measured by the SOFA score.

- Factor 11: Corresponds to the CNS function measured by the SOFA score and the number of organs in dysfunction. 
- Factor 12: Related to the loci of Sepsis and whether the infection is polymicrobial or not.

- Factor 13: Corresponds to the APACHE II score and worst lactate levels.

- Factor 14: Relates the total number of organs in dysfunction.

The factors obtained with this method are coherent with the SOFA score as a description and measure of organ failure and dysfunction [2], combined with the management guidelines defined by the Surviving Sepsis Campaign [7]. Therefore, it can be safely concluded that they are related to SOFA and the actions taken to mitigate this organ deterioration.

This is a result of particular interest. One of the main challenges in mortality prediction is that of producing flexible models that can robustly fit the observed data without the need for unnecessary contextual assumptions, and in the presence of subtle interactions between covariates. This happens because standard medical indicator-based models typically rely on hand-crafted parametric solutions to get around the problem [111]. One clear example of this is the categorization of the SOFA score prognostic indicators described in section 7.2. The obtained FA solution goes beyond this categorization while accounting for covariate interactions.

As mentioned in the introduction, the capability to interpret results is paramount in real clinical applications [107]. The reported FA not only complies with this requirement: it also provides a parsimonious data representation that can be used as a basis for mortality prediction related to the Sepsis pathology.

\subsubsection{Mortality prediction using logistic regression over 14 factors}

We now progress to the task of mortality prediction itself, using the obtained 14-factor FA solution as starting point. The performance of the model was evaluated by 10 -fold cross validation. Table 7.4 shows the coefficient estimates $\beta$, Z-Scores and maximum and minimum values resulting from fitting a logistic regression model to the 14 factors (inputs) and the outcome in the ICU (output) and removing those factors yielding Z-Scores smaller than 1.96. The Z-Scores measure the effect of removing one factor from the model [110, 81]. A Z-score greater than 1.96 in absolute value is significant at the $5 \%$ level and provides a measure of the relevance for the prediction of a given factor.

As shown in table 7.4, factor 3, which is related to Mechanical Ventilation and Pplateau, shows the strongest effect together with factor 13, which is related to the APACHE II score. Factor 8 (Hepatic Function measured with the SOFA Score) and factor 10 (related to the number of Dysfunctional Organs) are also found to be relevant. It is worth noting at this stage that, with LR, the factors related to the Surviving Sepsis Campaign show no strong effect on mortality prediction. This result may be due to the low compliance with the Surviving Sepsis Campaign Bundles for the first 6 and 24 hours of evolution $(26.18 \%$ and $44.06 \%$ respectively for the ICU under study). However, it is interesting to note that factor 9 (antibiotic administration and haemocultures) presents a higher impact than that of factor 6 ( $24 \mathrm{~h}$. bundles with glycaemic indexes). For our ICU, $80.22 \%$ of patients received antibiotics during the first $6 \mathrm{~h}$ of evolution and $77.14 \%$ had haemocultures during the same period of time. In fact, timely 
Table 7.3: Loadings Matrix: $|\Lambda(i, j)|>$ quantile 95 for Factor $f_{i}$ are presented in bold.

\begin{tabular}{|c|c|c|c|c|c|c|c|c|c|c|c|c|c|c|}
\hline & F1 & F2 & F3 & F4 & F5 & F6 & F7 & F8 & F9 & F10 & F11 & F12 & F13 & F14 \\
\hline v1 & .27 & -.12 & -.05 & .03 & -.11 & -.05 & .08 & -.04 & .10 & -.03 & .08 & -.16 & .22 & -.09 \\
\hline $\mathrm{v} 2$ & .00 & .02 & .14 & .13 & -.13 & .04 & .21 & .02 & .03 & $\begin{array}{l}-.01 \\
\end{array}$ & -.09 & -.09 & -.04 & -.04 \\
\hline v3 & .13 & .06 & -.32 & -.07 & .00 & .06 & .01 & -.04 & .08 & .19 & .07 & .42 & -.08 & .06 \\
\hline $\mathrm{v} 4$ & -.12 & -.03 & -.01 & .06 & -.8 & .98 & -.04 & -.03 & -.03 & -.05 & -.04 & -.03 & -.03 & -.04 \\
\hline $\mathrm{v} 5$ & $\begin{array}{l}-.01 \\
\end{array}$ & .05 & -.01 & .04 & .05 & .70 & .04 & -.07 & -.02 & $\begin{array}{l}-.01 \\
\end{array}$ & -.02 & .09 & -.04 & .03 \\
\hline $\mathrm{v} 6$ & .16 & -.21 & .23 & .13 & .05 & .06 & -.02 & -.05 & .05 & -.03 & -.07 & .47 & .03 & .13 \\
\hline $\mathrm{v} 7$ & .97 & .09 & -.03 & .15 & -.01 & -.03 & .09 & -.01 & .02 & .04 & .01 & .02 & .07 & .01 \\
\hline v8 & .08 & .03 & .86 & .38 & -.01 & .01 & .05 & .05 & -.05 & .12 & .06 & -.02 & -.10 & .09 \\
\hline v9 & .11 & -.01 & .00 & .09 & -.06 & -.05 & .01 & .03 & .06 & .05 & .95 & -.02 & .05 & -.01 \\
\hline v10 & .13 & .18 & .07 & .00 & .12 & -.04 & .10 & -.01 & .14 & .94 & .05 & .08 & .04 & -.01 \\
\hline v11 & .20 & .14 & -.04 & -.01 & -.03 & .01 & .89 & -.06 & .10 & .05 & .02 & .04 & .09 & .05 \\
\hline v12 & .14 & .97 & .04 & .05 & .03 & .02 & .04 & .00 & .04 & .10 & .02 & .10 & .07 & -.05 \\
\hline v13 & .61 & .37 & .27 & .20 & .03 & -.01 & .43 & -.01 & .11 & .32 & .26 & .09 & .01 & -.06 \\
\hline v14 & .01 & .23 & -.05 & -.11 & .03 & -.06 & .11 & .04 & .94 & .13 & .07 & .09 & .04 & -.01 \\
\hline v15 & .56 & .33 & .24 & .28 & -.02 & -.02 & .31 & .01 & -.36 & .23 & .28 & .04 & .08 & .26 \\
\hline v16 & .44 & .44 & .15 & .12 & .02 & $\begin{array}{l}-.06 \\
\end{array}$ & .33 & .04 & .48 & .30 & .28 & .09 & .11 & .21 \\
\hline v17 & .18 & .07 & .13 & .95 & -.04 & .03 & .08 & .05 & -.06 & .06 & .11 & -.05 & .01 & .02 \\
\hline v18 & .05 & -.10 & .82 & .04 & .04 & .02 & -.03 & -.08 & -.01 & -.03 & .02 & .17 & .06 & .04 \\
\hline v19 & .92 & .12 & -.06 & .13 & -.02 & .08 & .08 & .02 & -.01 & .03 & .01 & .03 & .02 & .04 \\
\hline $\mathrm{v} 20$ & -.08 & -.63 & -.03 & .00 & -.01 & .01 & -.16 & .04 & -.18 & -.03 & -.02 & .04 & .00 & -.08 \\
\hline $\mathrm{v} 21$ & .38 & .17 & .25 & .36 & -.09 & .03 & .40 & .04 & .05 & .09 & .23 & .05 & .46 & -.06 \\
\hline $\mathrm{v} 22$ & -.11 & -.02 & .15 & .01 & .03 & .08 & .00 & .52 & .09 & -.01 & .08 & .08 & .10 & -.07 \\
\hline $\mathrm{v} 23$ & .16 & .04 & .03 & -.07 & .02 & -.08 & .01 & .69 & -.05 & .05 & .06 & -.12 & -.06 & .02 \\
\hline v24 & .01 & -.03 & -.02 & .00 & .04 & -.10 & -.03 & .62 & .01 & -.01 & -.07 & -.11 & .02 & .02 \\
\hline v25 & .46 & .07 & -.04 & .10 & .10 & .05 & -.02 & .34 & .01 & -.03 & -.02 & .04 & .21 & -.03 \\
\hline v26 & -.10 & -.01 & -.02 & -.07 & -.11 & -.04 & -.05 & .05 & .07 & .11 & .11 & .09 & .04 & .01 \\
\hline $\mathrm{v} 27$ & .04 & -.10 & -.02 & .00 & .05 & -.03 & -.02 & .22 & -.01 & -.01 & .00 & -.41 & -.09 & .08 \\
\hline $\mathrm{v} 28$ & -.05 & .06 & .02 & -.08 & .00 & -.05 & -.04 & .04 & .01 & .07 & .05 & -.02 & -.01 & -.06 \\
\hline v29 & .09 & .24 & .03 & .18 & .06 & .06 & -.02 & .04 & $\begin{array}{l}-.01 \\
\end{array}$ & .07 & -.01 & -.06 & .06 & .12 \\
\hline v30 & .00 & .05 & -.04 & -.09 & .90 & -.07 & -.01 & .08 & -.01 & .05 & -.01 & .01 & .02 & -.02 \\
\hline v31 & .01 & -.05 & -.01 & .01 & -.10 & .98 & .02 & .05 & .07 & .09 & -.01 & -.01 & -.03 & -.10 \\
\hline v32 & -.15 & -.06 & -.18 & -.54 & .12 & -.04 & -.01 & .09 & .05 & .08 & .00 & -.09 & -.05 & .01 \\
\hline v33 & .28 & .20 & .11 & .21 & .04 & -.03 & .24 & .08 & -.04 & .19 & -.03 & .14 & .31 & .04 \\
\hline v34 & .21 & .11 & -.07 & -.02 & .04 & .07 & .00 & .24 & .05 & .02 & .04 & .03 & .31 & .03 \\
\hline
\end{tabular}


administration of antibiotics and performance of haemocultures are considered critical to improving the prognosis of septic patients.

Regression on the 14 factors together with 10-Fold cross validation resulted in an AUC of 0.78. A decision threshold of $\gamma=0.68$ was automatically selected (for the maximization of the discrimination probability) to decide whether the patient survives. This 10-fold cross-validation experiment yielded an AUC of 0.78 , an error rate of 0.24 , a sensitivity of 0.65 and a specificity of 0.80 . The results of LR over latent factors is presented in table 7.4. This table also shows that the two most representative factors are F10 and F13, which correspond to organ dysfunction measured through the SOFA score and illness severity measured through the APACHE II score combined with the worst lactate levels.

Table 7.4: Results for LR over Latent Factors with 10-fold cross validation

\begin{tabular}{|c|c|c|c|c|}
\hline & $\beta$ Coeff & MAX & MIN & Z-score \\
\hline Intercept & 1.22 & 1.53 & .87 & 7.11 \\
\hline F4 & -0.54 & -0.23 & -0.86 & -3.38 \\
\hline F10 & -0.69 & -0.38 & -1.05 & -4.26 \\
\hline F9 & -0.51 & -0.21 & -0.81 & -3.36 \\
\hline F13 & -0.49 & -0.24 & -0.74 & -3.80 \\
\hline
\end{tabular}

\subsubsection{Comparison with Logistic Regression over a Selec- tion of the Original Variables}

Further experiments aimed to compare the predictive ability of the FA 14-factor solution with that of the original data attributes were carried out. For that, the most significant clinical attributes were selected in a backward feature selection process (in our case, the backward feature selection removes those variables resulting in non-significative $\mathrm{Z}$-scores). The selected attributes were: the total number of dysfunctional organs; the APACHE II score; and the worst lactate levels. The corresponding coefficients, maximum and minimum values and Zscores for these three variables are presented in table 7.5 .

Table 7.5: Results for LR with 10-fold cross validation

\begin{tabular}{|c|c|c|c|c|}
\hline & $\beta$ Coeff & MAX & MIN & Z-score \\
\hline Intercept & 4.20 & 3.11 & 5.29 & 7.56 \\
\hline APACHE II & -0.08 & -0.13 & -0.04 & -3.77 \\
\hline Worts Lact. & -0.25 & -0.38 & -0.11 & -3.63 \\
\hline
\end{tabular}

Regression on the most significant attributes together with 10-fold cross validation yield an AUC of 0.75 , a lower result than the one obtained with the FA solution. Following the procedure outlined in the previous subsection, a decision threshold of $\gamma=0.68$ was automatically selected. This resulted in a prediction error over the test data of 0.3 (higher than the FA solution), a specificity of 0.72 , and a sensitivity of 0.64 . 


\subsubsection{Comparison with the APACHE II Mortality Score}

The Risk-of-Death (ROD) formula based on the APACHE II score can be expressed as [1]:

$$
\ln \left(\frac{R O D}{1-R O D}\right)=-3.517+0.146 \cdot A+\epsilon
$$

Where $A$ is the APACHE II score and $\epsilon$ is a correction factor depending on clinical traits at admission in the ICU. For instance, if the patient has undergone post-emergency surgery, $\epsilon$ is set to 0.613 . The application of this formula with a threshold of $\gamma=-0.25$ to the population under study yields an error rate of 0.28 (higher than the FA solution), a sensitivity of 0.82 and a specificity of 0.55 . The AUC was 0.70 .

A previous study [112] presented very similar results to those reported in this section for a similar ICU. Furthermore, a recent study from 2009 [113] presented very similar results to those reported here for neurocritically ill patients (with a very low sensitivity of 0.47 ).

\subsection{Conclusions}

Sepsis is a prevalent pathology in the clinical ICU environment, and one associated with relatively high levels of mortality. Its medical management is therefore both a sensitive issue and a serious challenge to health care systems.

The clinical indicators of Sepsis currently in use are known to be of limited relevance as mortality predictors. In the assessment of ROD for critically ill patients, sensitivity is important due to the fact that more aggressive treatment and therapeutic actions may result in better outcomes for high risk patients. As validated by the results reported in section 7.3.5 and similar ones reported in other studies [112], the ROD formula presented in [1] is very sensitive but also quite poor in terms of specificity (i.e., it results in a high number of false negative cases). This is despite the fact that it is widely accepted in practice and yields acceptable accuracy results. Its poor specificity may be the result of its formula being based on clinical traits and the APACHE II score only.

In this chapter, we have put forward a new and simple method for the assessment of ROD in septic patients. It proposes a change of data representation in the form of feature extraction using FA, and uses LR over the resulting latent factors for the prediction itself. The main advantage of the proposed approach is that it removes collinearities and noisy inputs while keeping the method simple and fully interpretable from a clinical point of view. In other words, the strength of this study lies in the fact that it is possible to derive a prognostic score from a set of physiopathologic and therapeutic variables, which are available at the onset of Severe Sepsis.

Although one might well object that it is easier to assess three variables than three factors (i.e. LR against Factor Analysis), we must stress the fact that the three factors obtained are actionable at ICU admittance whilst the worst lactate, which is the most predictive variable for LR is time consuming and may not take place at ICU admittance.

The proposed method may be understood as a generalization of the ROD formula introduced in [1], where the $\epsilon$ corrective factor, which models clinical traits at admittance in the ICU, is accounted for by the latent-factor representation. 
It takes not only the contribution of the APACHE II score into consideration, but also other important clinical traits such as the number of dysfunctional organs combined with the Sequential Organ Failure Assessment (SOFA), which also impacts on the mortality rates of Septic patients. The reported ROD assessment takes into consideration the Respiratory and Hepatic SOFA scores. It is precisely all the extra parameters considered in our experiments the reason behind the significant improvement on specificity if compared with the original specificity of the APACHE II score (i.e. 0.55). This improvement is achieved while keeping model complexity under control and without compromising the interpretation of the results (given that all the parameters involved are routinely monitored in an ICU).

A word of caution must be given, though, as the system performance has only been evaluated in a single ICU and limited population samples. For this reason, future work should lead toward a multi-centric prospective study, in order to validate the generalizability of the method. 


\section{Chapter 8}

\section{Severe Sepsis Mortality Prediction from Observed Data}

You know my methods. Apply them.

Sherlock Holmes

\subsection{Introduction}

So far we have focused on the study of dependence relations between the different variables and clinical traits and exploited its marginalisation to study the incidence of Severe Sepsis or its prognosis by means of Factor Analysis and Logistic Regression. We have been working on already interpretable indicators that could be used by the clinical practice.

In this approach we first embed the data in a suitable feature space, and then use algorithms based on linear algebra, geometry and statistics for inference. With this informal definition, it becomes apparent that all the methods used so far could be kernelized as long as we used the appropriate mappings, spaces, measures and topologies. Given the simplicity of the models used in this PhD (i.e. we only have multinomial and multivariate Gaussian distributions, which can be efficiently modelled algebraically by means of the Regular Exponential Family) we propose to use a generative approach and exploit the inner structure of our data in order to build a set of efficient closed-form kernels best suited for these two distributions (see sections 4.6 and 4.6.2).

For the experiments in this chapter, the database of patients of chapter 7 was available. It was used to investigate the performance of RVM and Generative Kernels as an ICU Sepsis Mortality Predictor. This performance was then compared to that of alternative techniques currently in use for ICU-related prediction, such as shrinkage methods for logistic regression and a risk-of-death (ROD) formula based on the standard APACHE II score [1]. The proposed models are shown to outperform these techniques, while simultaneously assessing the 
relative impact of individual indicators of the pathology on the prediction.

Interestingly, a number of these indicators, which are also readily interpretable, are shown to have an impact on mortality prediction. We believe that this is a result that should help to simplify the decision making process at ICU. This chapter uses the same dataset presented in chapter 7 .

\subsection{Materials: Detailed Description of Genera- tive Kernels}

In this section we present two of the main contributions of this $\mathrm{PhD}$ thesis: the Quotient Basis Kernel (QBK) and the simplified Fisher kernel. The methods presented in this section will be tested in the context of dimensionality reduction presented in chapter 5: RVM, the Lasso, ridge regression and logistic regression with backward feature selection. The set of attributes selected from these methods is later used with the kernels presented in this section.

\subsubsection{Quotient Basis Kernel}

In this section we use the definitions of algebraic models as presented in chapter 4. Inputs are denoted by $x$, responses or outputs are denoted by $y$ as in chapter 5, parametric functions are denoted by $\eta$ or functions of $\eta$. These are related by polynomial algebraic relations, possibly implicit (cf. section 4.2). Another feature of this definition is that constraints of polynomial type can be included in the specification of the model. Implicit models and the introduction of constraints can lead to the use of dummy variables.

The parameters of the model as interpreted in statistics are functions of any form with the restriction that they belong to a specified field. For example, $\mathbb{Q}\left(\eta_{1}, \ldots, \eta_{p}\right)$ is the set of all rational functions in $\eta_{1}, \ldots, \eta_{p}$ with rational coefficients. Another example is $\mathbb{Q}\left(e_{1}^{\eta}, \ldots, e_{p}^{\eta}\right)$ the set of all exponential rational functions. Parameters are treated as unknown quantities and in most cases appear in linear form. The algebraic space used is the commutative ring of all polynomials $\mathbb{K}\left[x_{1}, \ldots, x_{s}\right]$ in the indeterminates $x_{1}, \ldots, x_{s}$ and with coefficients in the field $\mathbb{K}$ (in our case $\mathbb{R}$ ).

For a given initial ordering, a term is specified by the vector of length $s$ of its exponents. Therefore Term $\{s\}$ is coded by $\mathbb{Z}_{+}^{s}[57]$ (set of positive integers).

When the indeterminates are indexed from 1 to $s$ so that $x_{1}, \ldots, x_{s}$, it is convention to consider an initial ordering $x_{i} \succ x_{i+1} \forall i=1 \ldots s-1$.

Definition 42. Polynomial Ideal (c.f. definition 12):

1. A polynomial ideal $I$ is a subset of a polynomial ring $\mathbb{K}[x]$ closed under sum and product by elements of $\mathbb{K}[x]$. Specifically the set $I \subset \mathbb{K}$ is an ideal if $\forall f, g \in I$ and $s \in \mathbb{K}$ the polynomials $f+g$ and sf are in $I$.

2. Let $F$ be a set of polynomials. The ideal generated by $F$ is the smallest ideal containing $F$. It is denoted $\langle F\rangle$.

3. An ideal $I$ is radical if $f \in I$ whenever a positive integer $m$ exists such that $f^{m} \in I$.

4. The radical of an ideal $I$ is the radical ideal defined as $\sqrt{I}=\left\{f \in \mathbb{K}: \exists m \mid f^{m} \in I\right\}$ 
The Hilbert basis theorem ([57]) shows that every ideal has a finite basis. This provides a very powerful result since it means that any ideal is finitely generated (even if the generating set is not necessarily unique). Another powerful result is that this generation basis is of a special type called Gröbner Basis, which we define below. This bases will become essential in the derivation of regression/interpolation polynomials and also for the algebraic derivation of the Fisher and the proposed QBK kernels.

Definition 43. [57] (c.f. definition 6) Let $\tau$ be a term ordering on $\mathbb{K}[x]$ and $f$ a polynomial in $\mathbb{K}[x]$. The leading term of $f, L T_{\tau}(f)$ is the largest term with respect to $\tau$ among the terms in $f$.

Definition 44. [57] Gröbner Basis (c.f. definition 14): Let $\tau$ be a term ordering on $\mathbb{K}[x]$. A subset $G=g_{1}, \ldots, g_{t}$ of an ideal $I$ is a Gröbner basis of I with respect to $\tau$ iff

$$
\left\langle L T_{\tau}\left(g_{1}\right), \ldots, L T_{\tau}\left(g_{t}\right)\right\rangle=\left\langle L T_{\tau}(I)\right\rangle
$$

where $L T_{\tau}(I)=\left\{L T_{\tau}(f): f \in I\right\}$.

Theorem 15. [63, 57] (c.f. theorem 4) Given a term ordering, every ideal I except $\{0\}$ has a Gröbner basis and any Gröbner basis is a basis of $I$.

Definition 45. Gröbner basis of unique points [63, 57] (c.f. section 4.2.3): Let $A$ be a set of $n$ unique points $A=\left\{\mathbf{a}_{\mathbf{1}}, \ldots, \mathbf{a}_{\mathbf{n}}\right\}$ and $\tau$ a term ordering. These points can be presented as the set of solutions of

$$
\left\{\begin{array}{c}
g_{1}(\mathbf{a})=0 \\
g_{2}(\mathbf{a})=0 \\
\cdots \\
g_{t}(\mathbf{a})=0
\end{array}\right.
$$

Where $G=g_{1}, \ldots, g_{t}$ is a Gröbner basis of $A$.

Let us formally define the Quotient Basis $\mathrm{EST}_{\tau}$ that shall be used in the algorithm below.

Definition 46. [57] Quotient Basis (c.f. definition 15):

Let $A$ be a set of unique support points $A=\left\{\mathbf{a}_{\mathbf{1}}, \ldots, \mathbf{a}_{\mathbf{n}}\right\}$ and $\tau$ a term ordering. A monomial basis of the set of polynomial functions over $A$ is

$$
E S T_{\tau}=\left\{x^{\alpha}: x^{\alpha} \notin\langle L T(g): g \in I(A)\rangle\right\}
$$

This definition states that $\operatorname{EST}_{\tau}$ comprises the elements $x^{\alpha}$ that are not divisible by any of the leading terms of the elements of the Gröbner basis of $I(A)$.

Theorem 16. [57] (c.f. theorem 5) The set EST $T_{\tau}$ has as many elements as there are support points.

Definition 47. Design Matrix 35 (c.f. definition 35)

Let $\tau$ be a term ordering and let us consider an ordering over the support points $A=\left\{\mathbf{a}_{\mathbf{1}}, \ldots, \mathbf{a}_{\mathbf{n}}\right\}$. Let $L$ be the set of exponents of $E S T_{\tau}$. We call design matrix the following matrix

$$
Z=\left[a_{i}^{\alpha}\right]_{i=1, \ldots, N, \alpha \in L}
$$


Theorem 17. [57] (c.f. theorem 14)

1. $Z$ is non-singular.

2. Let $e_{i}$ be the d dimensional canonical vector (i.e. with components 0 except in position $i$ where it has value 1 . For all $i=1, \ldots, d$ there exists a vector $c_{i}$ such that

$$
Z \cdot c(i)=e_{i}
$$

and the polynomial $\sum_{\alpha \in L} c_{i_{\alpha}} x^{\alpha}$ interpolates the indicator function of the support point $a_{i}$. That is

$$
\sum_{\alpha \in L} c_{i_{\alpha}} x^{\alpha}=\left\{\begin{array}{cc}
1 & x=a_{i} \\
0 & x \neq a_{i} \text { and } x \in A
\end{array}\right.
$$

Proposition 8. (c.f. proposition 3) The covariance of $Z$,

$$
\left.\operatorname{cov}(Z)=E(Z-E(Z))(Z-E(Z))^{t}\right)
$$

is a kernel.

Definition 48. Quotient Basis Kernel (QBK) (c.f. corollary 1):

The covariance of the design matrix of $E S T_{\tau}$, which is a kernel, is the QBK.

The algorithm for the calculation of $E S T_{\tau}$, which shall be used to calculate our QBK from the design matrix $Z$ is described in algorithm 1 . This algorithm was originally developed for the derivation of interpolation/regression polynomials in [63].

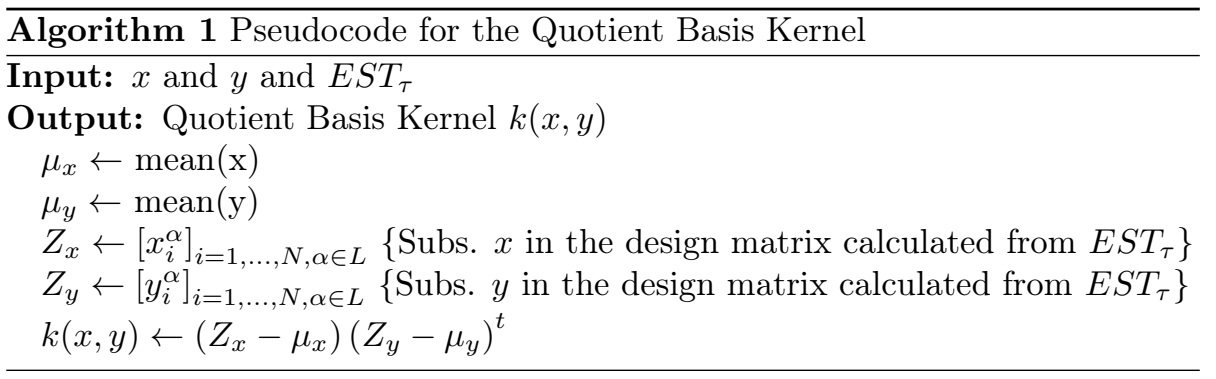

\subsubsection{Fisher Kernel for Exponential Families}

Lets us recall from section 4.6.2 that the computation of the Fisher Kernel is computationally expensive. Therefore, we propose to use the simplified (practical) Fisher Kernel from the sufficient statistics $(T)$ as defined below:

Definition 49. Practical Fisher Kernel

$$
k(x, z)=U\left(\bar{T}_{x}, \eta\right) U\left(\bar{T}_{z}, \eta\right)^{t}
$$

Where $T_{x}$ and $T_{z}$ are the sufficient statistics estimated on $x$ and $z$.

Here $U\left(\bar{T}_{x}, \eta\right)$ is the score function as defined in section 4.6.2. The product of distances of each point can be understood as a further simplification of the method presented in [114], which approximates distances between gradients (see section 4.6) through a stochastic selection rule. 


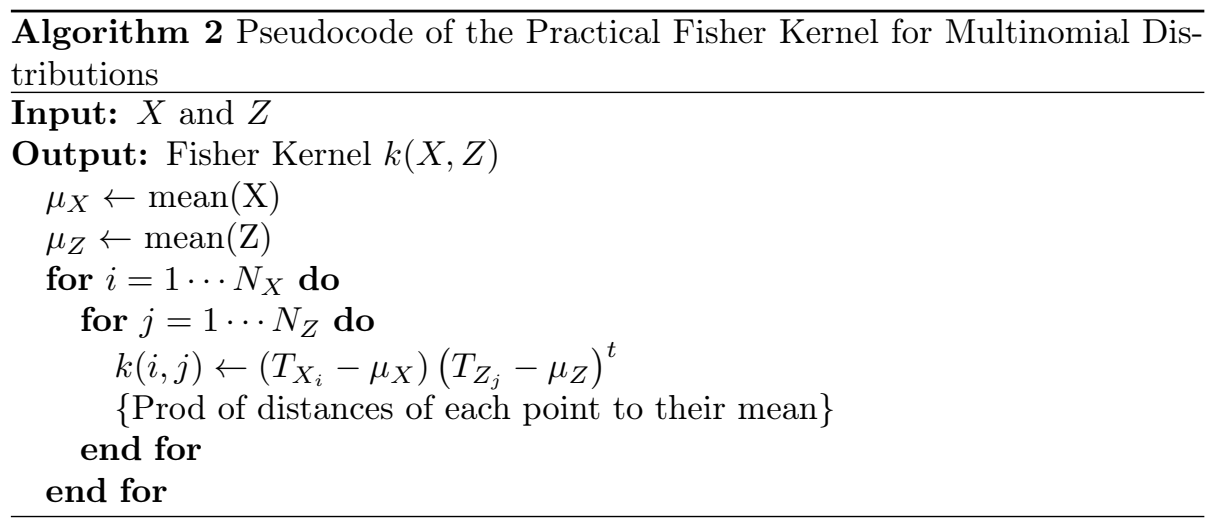

\subsubsection{Kernels based on the Jensen-Shannon metric}

The Kernels based on the Jensen-Shannon metric have been formally presented in section 4.6.3. For the sake of clarity, here we give a short overview about the propositions that yield these generative kernels.

Definition 50. [76, 77\%, 78]

Let $\gamma_{1}, \gamma_{2} \in M$ (parameters in dual space) and $F$ the dual of the cummuluant generating function $G$, by definitions 39, 40 and A.10:

$$
J S\left(\gamma_{1}, \gamma_{2}\right)=\frac{F\left(\gamma_{1}\right)+F\left(\gamma_{2}\right)}{2}-F\left(\frac{\gamma_{1}+\gamma_{2}}{2}\right)
$$

Proposition 9. [76, 777, 78] Centred Kernel

By property 8 and definition 40, let $x_{0} \in X$ define the centred kernel as $\phi: X \times X \rightarrow \mathbb{R}$

$$
\phi(x, y)=J S\left(x, x_{0}\right)+J S\left(y, x_{0}\right)-J S(x, y)-J S\left(x_{0}, x_{0}\right)
$$

Proposition 10. [76, 77, 78] Exponentiated Kernel

By property 9 and definition 40, we define the exponentiated kernel as $\phi$ : $X \times X \rightarrow \mathbb{R}$

$$
\phi(x, y)=\exp (-t J S(x, y))
$$

$\forall t>0$.

Proposition 11. [76, 77\%, 78] Inverse Kernel

By proposition 10 and definition 40, we define the exponentiated kernel as $\phi: X \times X \rightarrow \mathbb{R}$

$$
\phi(x, y)=\frac{1}{t+J S(x, y)}
$$

$\forall t>0$.

It is obvious that the most important part to calculate the kernels outlined above is the calculation of the Jensen-Shannon metric in dual-space. The pseudocode to implement this metric is given in algorithm 3 . 

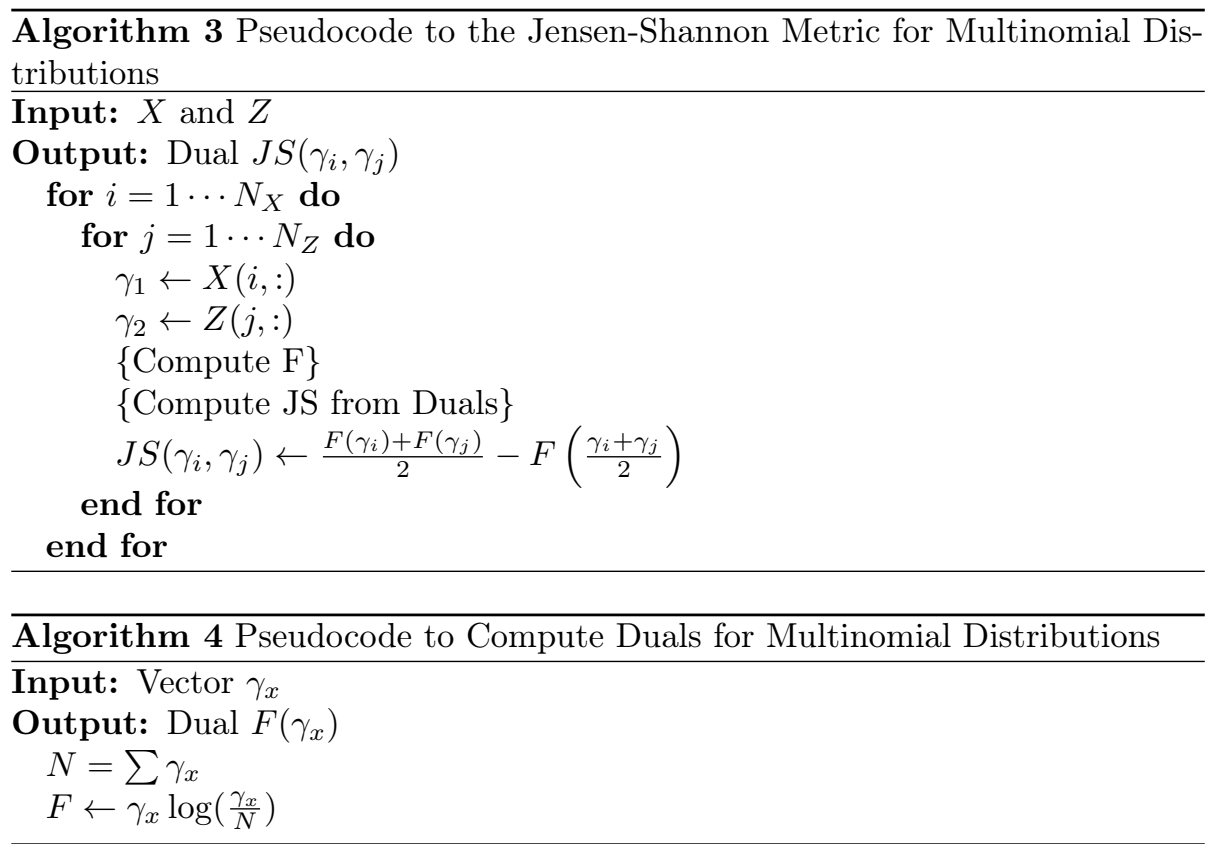

\subsection{Results}

\subsubsection{Mortality Prediction with RVM}

The model performance was evaluated by means of 10 -fold cross-validation. The RVM yielded an accuracy of mortality prediction of 0.86 as measured by the area under the ROC plot (AUC); a prediction error of 0.18; a sensitivity (proportion of correctly predicted survivors out of all survivors) of 0.67 ; and a specificity (proportion of correctly predicted exitus out of all exitus) of 0.87 .

Beyond classification accuracy, and as described in the previous section, RVM performs soft feature selection through automatic feature relevance determination. The following relevance vector (with the weights associated to each input feature) was obtained:

- Number of dysfunctional organs $\left(w_{1}=-0.039\right)$

- Mechanical Ventilation $\left(w_{2}=-0.101\right)$

- $\mathrm{APACHE}$ II $\left(w_{3}=-0.337\right)$

- Resuscitation Bundles (6h) $\left(w_{4}=0.037\right)$

The coefficients corresponding to the rest of features were set by RVM to zero (i.e. lower than the numeric tolerance set in Matlab: $2.2 \times 10^{-16}$ ) as part of the training process. This effectively reduces the complexity of the prediction procedure (34 features reduced down to just 4) and consequently, improves its interpretation. Given that a linear basis function was used to estimate the relevance vector, it becomes apparent that the negative weights (number of dysfunctional organs, mechanical ventilation, APACHE II) are related to a higher mortality risk (note again that we have coded survival as 1 and exitus as -1), 
whereas the SSC bundles (resuscitation bundles) are associated to a protective effect (i.e. antibiotics administration, performance of haemocultures, administration of volume and vasoactive drugs and so on). In fact, timely administration of antibiotics and performance of haemocultures are considered critical to improving the prognosis of septic patients. Equally important is the knowledge of which features are deemed not to be relevant by RVM.

The set of variables selected by the RVM also present clinical relevance since they are widely used in clinical practice for the assessment of ROD [1, 25, 7]. Of particular interest are the SSC bundles due to the relevant scientific information supporting them [7]. It is this subset of variables selected by the RVM that shall be used in the next sections of this chapter.

\subsubsection{Comparison with Shrinkage Feature Selection Meth- ods for Logistic Regression}

The predictive ability of the RVM was then compared to that of other well established shrinkage methods for logistic regression. In particular, we have tested the performance against Ridge Regression, the Lasso and Logistic Regression. The latter using a subset of features selected in a backward process by removing those coefficient yielding the lowest Z-scores [81]. The selected features and coefficients for each method were:

- Ridge Regression:

- Number of dysfunctional organs for SOFA 3-4 $\left(w_{1}=-0.021\right)$

- APACHE II $\left(w_{2}=-0.127\right)$

- Worst Lactate $\left(w_{3}=-0.126\right)$.

- Lasso:

- Age $\left(w_{1}=0.007\right)$

- Germ Class $\left(w_{2}=0.005\right)$

$-\mathrm{PaO}_{2} / \mathrm{FiO}_{2}\left(w_{3}=0.001\right)$

- $\operatorname{APACHE}$ II $\left(w_{4}=-0.006\right)$

$-\mathrm{SvcO}_{2} 6 \mathrm{~h}\left(w_{5}=-0.001\right)$

- Haematocrit $6 \mathrm{~h}\left(w_{6}=0.009\right)$

- Worst Lactate $\left(w_{7}=-0.023\right)$

$-\mathrm{SvcO}_{2}\left(w_{8}=-0.006\right)$.

- Logistic Regression with backward feature selection:

- Intercept $\left(w_{1}=4.20\right)$

- Number of Dysfunctional Organs $\left(w_{1}=-0.12\right)$

- APACHE II $\left(w_{2}=-0.08\right)$

- Worst Lactate $\left(w_{3}=-0.25\right)$ 
The three shrinkage methods evaluated in this section agreed in detecting as prognostic factors the Severity measured by the APACHE II score and acidosis measured by the lactate levels. Apart from that, it becomes apparent that organ dysfunction and mechanical ventilation or other parameters related to it like $\mathrm{PaO}_{2} / \mathrm{FiO}_{2}$ also play a role in the prognosis of Sepsis. Table 8.1 shows the results of AUC, error rate, sensitivity and specificity for each method. So far we have tested different approaches to the study of the prognosis of sepsis ranging from dimensionality reduction algorithms like Factor Analysis to Shrinkage Methods like Ridge Regression and the Lasso. In this section we have shown that application of the RVM outperforms all the methods outlined so far in terms of AUC and specificity.

Table 8.1: Results for Shrinkage Methods

\begin{tabular}{|c|c|c|c|c|}
\hline Method & AUC & Error Rate & Sens. & Spec. \\
\hline RVM & 0.86 & 0.18 & 0.67 & 0.87 \\
\hline Logistic & 0.75 & 0.30 & 0.64 & 0.72 \\
\hline Ridge & 0.70 & 0.25 & 0.63 & 0.79 \\
\hline Lasso & 0.70 & 0.32 & 0.67 & 0.68 \\
\hline
\end{tabular}

\subsubsection{Mortality Prediction with Generative Kernels}

The different kernels have been implemented in Matlab following the algorithms and propositions outlined above.

The calculation of the Quotient Basis Kernel required the implementation of the algorithm outlined above to calculate $\mathrm{EST}_{\tau}$ with the lexicographic ordering.

The input to algorithm were the unique points of our input data for each of the four variables of interest selected by the RVM (i.e. all the observed combination of points from the input) ${ }^{1}$. Here

- $x_{1}$ is the Number of Dysfunctional Organs as measured by the SOFA Score.

- $x_{2}$ corresponds to Mechanical Ventilation (yes/no).

- $x_{3}$ corresponds to Severity as Measured by the APACHE II Score.

- $x_{4}$ corresponds to the SSC Resuscitation Bundles (i.e. administration of antibiotics, performance of haemocultures and so on). This is also a binary variable.

The resulting Quotient Basis $\mathrm{EST}_{\tau}$ for our dataset is

\footnotetext{
${ }^{1}$ The rationale behind selecting this subset is that not only has been automatically generated but is also in good agreement with common clinical practice since it balances organ dysfunction with timely administration of antibiotics
} 


$$
\mathrm{EST}_{\tau}=\left\{\begin{array}{c}
1, x_{4}, x_{3}, x_{3} x_{4}, \\
x_{2}, x_{2} x_{4}, x_{2} x_{3}, x_{2} x_{3} x_{4}, \\
x_{2}^{2}, x_{2}^{2} x_{4}, x_{2}^{2} x_{3}, x_{2}^{2} x_{3} x_{4}, \\
x_{2}^{3}, x_{2}^{3} x_{4}, x_{2}^{3} x_{3}, x_{2}^{3} x_{3} x_{4}, \\
x_{2}^{4}, x_{2}^{4} x_{4}, x_{2}^{4} x_{3}, x_{2}^{4} x_{3} x_{4}, \\
x_{2}^{5}, x_{2}^{5} x_{4}, x_{2}^{6}, x_{1}, \\
x_{1} x_{4}, x_{1} x_{3}, x_{1} x_{3} x_{4}, x_{1} x_{2}, \\
x_{1} x_{2} x_{4}, x_{1} x_{2} x_{3}, x_{1} x_{2} x_{3} x_{4}, x_{1} x_{2}^{2}, \\
x_{1} x_{2}^{2} x_{4}, x_{1} x_{2}^{2} x_{3}, x_{1} x_{2}^{2} x_{3} x_{4}, x_{1} x_{2}^{3}, \\
x_{1} x_{2}^{3} x_{4}, x_{1} x_{2}^{3} x_{3}, x_{1} x_{2}^{3} x_{3} x_{4}, x_{1} x_{2}^{4}, \\
x_{1} x_{2}^{4} x_{4}, x_{1} x_{2}^{4} x_{3}, x_{1} x_{2}^{5}
\end{array}\right\}
$$

The Quotient Basis Kernel is calculated by taking the Covariance after transforming the input points with $\mathrm{EST}_{\tau}$. Regarding interpolation, our sample space has 2016 unique points (i.e. $7 \times 2 \times 72 \times 2$ corresponding to the possible number of dysfunctional organs, mechanical ventilation, APACHE II and SSC resuscitation bundles). In our database, we have 354 different patients with a $7.63 \%$ repeated samples (i.e. 327 unique independent points). This means that we only have $16.22 \%$ of the available sample set.

At this stage, it is important to note that this Quotient Basis accounts for all the interactions between the different input variables. From section 4.4.2, this would mean that the four variables are conditionally dependent and also that this data can be represented by means of a fully connected graph. This interpretation is consistent with standard clinical practice.

Besides that, we have used Matlab's Support Vector Machine QP solver implemented in the BioInformatics and Optimization Toolboxes. We have also used 10-fold cross validation to evaluate the classification performance for the different kernels and also compare with the results presented in other chapters of this PhD Thesis. A grid search yielded the appropriate values for $C$ (c.f. section 5.2.2) parameters for each Kernel. More particularly,

- Quotient Basis and Fisher $C=1$.

- Generative Kernels $C=10$. Also the parameter $t$ for the Exponential and Inverse Kernels was set to 2.

- Gaussian, Linear and Polynomial Kernels $C=10$.

Statistical significance between errors has been tested by means of the Wilcoxon Rank Sum Test. The null hypotheses that we tested is whether the the errors are independent samples from identical continuous distributions with equal medians [115]. This test accepted the null hypothesis in all cases; the p-values for this test are given in table 8.3. Of course, the level of agreement measured by the p-value differs between the different kernels.

From table 8.2, it becomes apparent that there is no significant difference in performance between the most widely used kernels (Gaussian/Multivariate, Polynomial and Linear) as opposed to the four Generative Kernels tested. Moreover, all generative kernels yielded a good balance between AUC, sensitivity and specificity. However, from our results, it is also apparent that the Fisher kernel and the Quotient Basis kernel yield the best results (i.e. best error rate, AUC and balance between sensitivity and specificity). Table 8.2 also shows the 
average time taken to compute each kernel and train the SVM for the given dataset.

Table 8.2: Results for SVM with Generative Kernels

\begin{tabular}{|c|c|c|c|c|c|}
\hline Kernel & AUC & Error Rate & Sens. & Spec. & CPU time $[\mathrm{s}]$ \\
\hline Quotient & 0.89 & 0.18 & 0.70 & 0.86 & 1.45 \\
\hline Fisher & 0.76 & 0.18 & 0.68 & 0.86 & 1.39 \\
\hline Exponential & 0.75 & 0.21 & 0.70 & 0.82 & 1.64 \\
\hline Inverse & 0.62 & 0.22 & 0.70 & 0.82 & 1.68 \\
\hline Centred & 0.75 & 0.21 & 0.70 & 0.82 & 1.99 \\
\hline Gaussian & 0.83 & 0.24 & 0.65 & 0.81 & 1.56 \\
\hline Poly (order 2) & 0.69 & 0.28 & 0.71 & 0.76 & 1.59 \\
\hline Linear & 0.62 & 0.26 & 0.62 & 0.78 & 1.35 \\
\hline
\end{tabular}

Table 8.3: p-value table for the Wilcoxon Rank Sum Test. The null hypothesis tested is that the cdf for the resulting error distributions for each kernel are different

\begin{tabular}{|c|c|c|c|c|c|c|c|c|}
\hline & Quotient & Fisher & Exp & Inv & Cent & Gauss & Lin & Poly \\
\hline Quotient & $\mathrm{X}$ & 0.91 & 0.78 & 0.70 & 0.57 & 0.30 & 0.57 & 0.52 \\
\hline Fisher & & $\mathrm{X}$ & 0.82 & 0.60 & 0.42 & 0.91 & 0.60 & 0.67 \\
\hline Exp & & & $\mathrm{X}$ & 0.49 & 0.35 & 0.83 & 0.30 & 0.52 \\
\hline Inv & & & & $\mathrm{X}$ & 0.51 & 0.47 & 0.67 & 0.38 \\
\hline Cent & & & & & $\mathrm{X}$ & 0.42 & 0.27 & 0.17 \\
\hline Gauss & & & & & & $\mathrm{X}$ & 0.41 & 0.67 \\
\hline Lin & & & & & & & $\mathrm{X}$ & 0.41 \\
\hline Poly & & & & & & & & $\mathrm{X}$ \\
\hline
\end{tabular}

\subsection{Conclusions}

In the assessment of ROD for critically ill patients, sensitivity is important due to the fact that more aggressive treatment and therapeutic actions may result in better outcomes for high risk patients. As validated by the results reported in section 7.3.5 and similar ones reported in other studies [112], the ROD formula presented in [1] is poor in terms of sensitivity (i.e., it results in a high number of false negative cases). This is despite the fact that APACHE is widely accepted in practice and yields acceptable accuracy results. Its poor sensitivity may be the result of its formula being based on non-sepsis specific clinical traits and the APACHE II score only.

In this chapter, we have put forward an RVM-based method for the prediction of ROD in septic patients. It has been shown to produce accurate results, 
particularly in terms of specificity, while improving the interpretation and actionability of the results through an embedded feature relevance determination process. This method has proven to be superior in terms of accuracy (error rate, specificity and AUC) than other well established shrinkage methods (Lasso and Ridge). Specifically from a medical viewpoint, the strength of this study lies in the fact that it shows that it is possible to derive a reliable prognostic score from a parsimonious set of physiopathologic and therapeutic variables, which are available at the onset of severe sepsis for medical experts at the ICU.

The SVMs have been trained with eight different kernels out of which five were generative and the other three are kernels considered well suited for the problem at hand. Regarding the generative kernels, one is completely new (i.e. the Quotient Basis kernel) while the Fisher kernel has been derived by means of a combination of Algebraic Models and well established properties from the Regular Exponential Families.

The kernels proposed have proven to provide accurate and actionable results whilst keeping an acceptable balance between the different parameters of interest (AUC, error rate, sensitivity and specificity). In particular, the newly proposed Quotient Basis Kernel provided the most accurate results and almost equivalent to those of the the Fisher kernel in terms of balance between sensitivity and specificity (i.e. good proportion between positives and negatives). However, a Wilcoxon rank sum shows that all results are statistically equivalent.

The proposed methods may be understood as a generalization of the ROD formula introduced in [1], where the $\epsilon$ corrective factor, which models clinical traits at admittance in the ICU. The indicators obtained not only take the contribution of the APACHE II score into consideration, but also other important life-threatening clinical traits such as the number of dysfunctional organs combined with mechanical ventilation (RVM) or worst lactate levels (shrinkage methods). The prognosis indicator is also balanced with important procedures to overcome sepsis such as the administration of volume, antibiotics, vasoactive drugs and the performance of haemocultures (i.e. SSC resuscitation bundles). 


\section{Chapter 9}

\section{Conclusions}

Good reasons must, of force, give place to better.

William Shakespeare

In the previous chapters, we have first defined the general problem of Sepsis data analysis in the ICU environment and we have then focused our attention on some of the main challenges it involves, including the estimation of the incidence of sepsis, the prediction of ICU outcome for patients with Severe Sepsis and the impact of the pre-administration of statin drugs on such outcomes. To address these problems, we employed a wide array of techniques from the fields of multivariate and algebraic statistics, algebraic geometry, machine learning and computational intelligence. More specifically, ASMs have set the basis for the estimation within the geographic ambit of the study of the incidence of Sepsis. This has been accomplished using the Hammersley-Clifford theorem, which has enabled us to study this incidence as a hidden variable in a Bayes Network.

One of the main limitations of the quantitative methods for the assessment of Risk of Death currently in use at the ICU is their lack of specificity (i.e. the high number of false positive cases they incur), which not only puts an extra risk on an already severely affected patient population, but also results in an unnecessary burden for National Health Systems. In this regard, it has been shown that Machine Learning and related techniques can play an important role as they improve the overall performance by combining those indicators already in place with other clinical variables, which are routinely measured (even if not commonly used as indicators) such as the Surviving Sepsis Campaign Resuscitation Bundles (i.e. timely administration of antibiotics, performance of haemocultures and volume administration if necessary).

In this thesis this problem of ICU outcome prediction has been addressed according to two general approaches. The first involved a transformation of the originally observed data variables into new hidden or latent features that can be interpreted in medical terms and thus be used as new clinical indicators. The second involved using the original measurements in analyses that applied several strategies involving classification and dimensionality reduction. They included the use of classifiers such as logistic regression (common practice in 
the medical field), SVMs and RVMs. The latter related to techniques of feature selection (Ridge Regression or the Lasso) that have also been used with some of the classifiers. Even if different feature selection methods resulted in different subsets of selected variables, all of them pointed towards the same physiological systems (for example acidosis or mechanical ventilation parameters) and organ dysfunctions.

Attending to the nature of the indicators and clinical traits used in the medical practice, we have further built upon the ASM by relating them to the Regular Exponential Family. This can be intuitively understood as the means to re-parametrize a given support and under a given family (the multinomial distribution is also a Regular Exponential Family), in order to obtain a convex dual that simplifies the kernel generation. Another important result that we used is that this convex-dual is the Entropy Function (for the multinomial family this it is related to the relative frequencies), which can be calculated more efficiently. We have also used the ASM methodology to derive a new kernel (Quotient Basis Kernel), that is closely related to the Graphical Models presented in this PhD Thesis.

\subsection{On the Incidence of Sepsis and Coadjutant Factors to be Taken into Consideration}

Since its inception, the SIRS pathology has proven to be a sensitive indicator of Sepsis [43], but also one of poor specificity. For example, Pittet et al. [44] presented a SIRS incidence of up to $93 \%$ in critical care patients, while Rangel et al. have shown an incidence of $68 \%$ [43]. The latter study also shows that $25 \%$ of patients with SIRS developed Sepsis, $18 \%$ presented Severe Sepsis, and $4 \%$ of them, Septic Shock. This of course does not tell us much about the real number of Septic cases each year. In the case of Spain (where the data for this thesis was acquired), there is a clear and difficult to explain discrepancy between the incidence rates reported by hospitals in different regions. For example, Castilla y León reports 250 cases / year, while Madrid reports 141 cases / year ${ }^{1}$. The Bayes Network that we have presented in Chapter 6 was trained with the data from a prospective study at Hospital Vall d'Hebron, which is a hospital of similar size to the main ones in Madrid (i.e. Third Level Reference Hospital). This Bayes Network yielded an estimation of 164 cases/year.

A note of caution must be issued: We have to bear in mind that there are different comorbidities and coadjutant factors that clearly play a role in the onset and evolution of Sepsis. The most obvious one is whether the patient has undergone surgery or not prior to developing Sepsis. However, the role of many coadjutant factors in the development and prognosis of Sepsis is still controversial.

${ }^{1}$ This data is based on retrospective studies and, therefore, incidence is assessed a posteriori. 


\subsection{Summary of Prognosis Indicators Obtainend and Their Accuracy}

In this thesis, we have focused on the study of the role of the pre-admission use of statins in the incidence of Septic Shock and the prognosis of Sepsis (c.f. sections 6.4.3, 6.4.4 and 6.4.5). This has been studied using Graphical Models, Regression Trees and classification techniques. First, we have shown that there exists a dependency between the preadmission use of statins and the outcome of Sepsis. Moreover, we have seen that this dependence is much stronger if the severity level of the pathology and organ dysfunction are both taken into consideration. Our work has also shown that statins do not play a role in the incidence of Septic Shock. In fact, patients that received statins treatment presented a higher incidence of Septic Shock. However, it is also clear that for high severity levels and high organ dysfunctions, the patients that received statins treatment presented sensibly higher survival rates. We strongly believe that the discrepancies and controversy that we have seen in the literature may be due to this fact (i.e. differences of outcome according to Severity and Organ Dysfunction). Therefore, we are in a position to strongly recommended further randomized clinical studies to confirm whether the statins administration treatment should be continued during an ICU stay.

\subsection{Summary of Mortality Predictors and Their Accuracy}

As stated above, one of the main limitations of the current indicators for scoring the evolution of Sepsis is their lack of specificity. In this thesis, we have investigated 17 different approaches for the estimation of the Risk of Death (ICU outcome) and compared them with the standard APACHE II score. Table 9.2 summarizes the corresponding results for (in chronological order of development as presented in Chapters 7 and 8 ). This table shows the models proposed outperform the APACHE II score in terms of specificity.

The RVM (chapter 8) yielded an acceptable performance in terms of AUC, sensitivity and specificity, using a very parsimonious subset of indicators (very practical in clinical ease of use terms). This is more apparent if compared with other classification/feature selection methods like Logistic Regression (LR) with backward feature selection, Ridge Regression and the Lasso. The subset of input variables resulting from RVM were used to develop several generative kernels. Shrinkage is not only important to remove redundant information (and, therefore, improve performance), but also to keep computational complexity at bay. At this stage it is important to note that the attributes used for Logistic Regression over latent factors (chapter 7) uses the latent factors related to Mechanical Ventilation, Hepatic function, number of dysfunctional organs and the APACHE II score. The attributes that were selected by means of backward feature selection were the number of dysfunctional organs, the APACHE II and Worst Lactate Levels (this is the most expensive attribute to calculate since it requires the performance of periodic blood tests to assess its time evolution to obtain its worse levels). At last but not the least, the most predictive attributes found by the RVM were the number of dysfunctional organs, Mechanical Ventilation, 
APACHE II and the SSC resuscitation bundles. It is this final set of attributes that were used to implement the generative kernels. The different sets of attributes have been respectively labeled as FA (Factor Analysis), LR (Logistic Regression), and RVM (chapter 8). In table 9.1 we show a summary of all these attributes as well as whether these are calculated at ICU admittance (once) or periodically.

Table 9.1: Summary of attributes, the dataset where they are used and their calculation.

\begin{tabular}{|c|c|c|}
\hline Attribute & Dataset & Calculation \\
\hline Mechanical Vent. & FA/RVM & Admit. \\
\hline Hepatic Func. & FA & Admit. \\
\hline Num. Dysf.Org & FA/LR/RVM & Admit. \\
\hline APACHE II & FA/LR & Admit. \\
\hline Worst Lactate & LR & Periodic \\
\hline SSC Res. Bundles & RVM & Admit. \\
\hline
\end{tabular}

Regarding the generative kernels, they all yielded a good balance between AUC, sensitivity and specificity. It is also apparent that the Quotient Basis and Fisher kernels yielded the best results (i.e. best AUC and best balance between sensitivity and specificity) for the generative approach.

In conclusion, if we were to choose a method for assessing ROD, we would either choose RVM with Gaussian priors or an SVM with the Quotient Basis or Fisher Kernel since we believe that their computational cost pays-off in terms of accuracy whilst keeping the methods interpretable. In particular, the Quotient Basis Kernel can be represented by means of Graphical models. However, if we seek further simplicity interpretability and actionability (i.e. without having to wait for laboratory results), then the best option would be the Logistic Regression over Latent Factors proposed in this $\mathrm{PhD}$ thesis as shown in table 9.2 , which also shows an acceptable error rate. 
Table 9.2: Summary of Prognosis Indicators and their Corresponding Accuracies

\begin{tabular}{|c|c|c|c|c|c|}
\hline Method & AUC & Error Rate & Sens. & Spec. & Dataset \\
\hline LR-FA & 0.78 & 0.24 & 0.65 & 0.80 & FA \\
\hline LR & 0.75 & 0.30 & 0.64 & 0.72 & LR \\
\hline APACHE II & 0.70 & 0.28 & 0.82 & 0.55 & N/A \\
\hline RVM & 0.86 & 0.18 & 0.67 & 0.87 & RVM \\
\hline Ridge & 0.70 & 0.25 & 0.63 & 0.79 & RVM \\
\hline Lasso & 0.70 & 0.32 & 0.67 & 0.68 & RVM \\
\hline SVM-Quotient & 0.89 & 0.18 & 0.70 & 0.86 & RVM \\
\hline SVM-Fisher & 0.76 & 0.18 & 0.68 & 0.86 & RVM \\
\hline SVM-EXP & 0.75 & 0.21 & 0.70 & 0.82 & RVM \\
\hline SVM-INV & 0.62 & 0.22 & 0.70 & 0.82 & RVM \\
\hline SVM-CENT & 0.75 & 0.21 & 0.70 & 0.82 & RVM \\
\hline SVM-GAUSS & 0.83 & 0.24 & 0.65 & 0.81 & RVM \\
\hline SVM-LIN & 0.62 & 0.26 & 0.62 & 0.78 & RVM \\
\hline SVM-POLY & 0.69 & 0.28 & 0.71 & 0.76 & RVM \\
\hline
\end{tabular}

\subsection{Contributions}

\subsubsection{Methodological Contributions}

This $\mathrm{PhD}$ has resulted in the following methodological contributions:

1. The application of Algebraic Models and the study of Quotient Basis resulted in the definition of the Quotient Basis Kernel. This kernel has provided actionable and interpretable results for the assessment of ROD in Severe Sepsis. Also the structure of the Quotient Basis provides valuable information about the structure of the graphical model underlying our data. Unfortunately, our problem is quite unforgiving since all variables are interdependent (i.e. all our datasets yield fully connected graphs).

2. We have also shown that Maximum Likelihood inference of parameters for Regular Exponential Families under the ASM methodology can also be addressed as the minimization of a Bregman Divergence as in standard theory. Also the Bregman Divergence minimization over the convex dual can be done by means of Algebraic Methods for the Regular Exponential Family. This methodology has been used to derive the Generative Kernels presented in this $\mathrm{PhD}$ thesis with the clear objective of keeping the maximum interpretability of the relations between input variables.

\subsubsection{Clinical Contributions}

This $\mathrm{PhD}$ has resulted in the following clinical contributions: 
1. We have provided a set of actionable ROD indicators for Severe Sepsis, which are readily interpretable and actionable. We have also recommended to study and evaluate these indicators in different ICUs to guarantee their generalization.

2. We have also shown for the first time that the impact of preadmission use of Statins for septic patients is closely related to severity and organ dysfunction. This is considered to be one of the main reasons for the disparity of results found in the literature.

\subsection{Publications}

\subsubsection{Publications Directly Linked to this PhD Thesis}

This $\mathrm{PhD}$. thesis has resulted in the following list of publications:

- Ribas, V., Ruiz-Rodríguez, J.D., Wojdel, A., Caballero-López, J., RuizSanmartín A., Rello, J. and Vellido, A. Severe sepsis mortality prediction with Relevance Vector Machines. In Procs. of the 33rd Annual International Conference of the IEEE Engineering in Medicine and Biology Society (EMBC 2011).

- Ribas, V.J., Caballero-López, J., Saez de Tejada, A., Ruiz-Rodríguez, J.C., Ruiz-Sanmartín, A., Rello, J., Vellido, A. Graphical models for ICU outcome prediction in sepsis patients treated with statin drugs, In Procs. of the Eigth International Meeting on Computational Intelligence Methods in Bioinformatics and Biostatistics, (CIBB 2011).

- Ribas, V., Caballero-López, J., Ruiz-Rodríguez, J.C., Ruiz Sanmartín, A., Rello, J., and Vellido, A. On the use of decision trees for ICU outcome prediction in sepsis patients treated with statins. In Procs. of the IEEE Symposium Series on Computational Intelligence / IEEE Symposium on Computational Intelligence and Data Mining (IEEE SSCI CIDM 2011), pp.37-43.

- Ribas, V.J, Vellido, A., Ruiz-Rodríguez, J.C., Intelligent Management of Sepsis in the Intensive Care Unit in Intelligent Data Analysis for Real-Life Applications: Theory and Practice, IGI pub., in press.

\subsubsection{Relevant Information Related to this PhD Thesis}

- Intensive Care Conferences:

- Caballero López J.,Ruiz Rodríguez J.C., Sola-Morales O., Ribas Ripoll V., Ruiz Sanmartin A., Innovative continous non invasive cuffless blood pressure monitoring based on plethysmography technology, SCCM's 41st Critical Care Congress, Accepted.

- Ruiz Rodríguez J.C., Ribas Ripoll V., Monte Moreno E., Caballero López J., Francisco Salas E., Ruiz Sanmartin A., Martinez Pozo J.M., Delgado Tellez de Cepeda A.M., Bóveda Treviño J.L., "Validación de un nuevo indicador de predicción precoz de mortalidad en la Sepsis 
grave.", XLV Congreso Nacional de la SEMICYUC, XXXVI Congreso Nacional de la SEMICYUC, 7-10 Jun., 2009.

- Martínez Pozo J., Ruiz Rodríguez J.C., Delgado Téllez de Cepeda A.M., Ribas Ripoll V., Monte Moreno E., Caballero López J., Francisco Salas E., Ruiz Sanmartín A., Bóveda Treviño J.L., "Evaluación de un punto de corte en la escala SOFA basal como factor predictor de mortalidad en la Sepsis grave", XLV Congreso Nacional de la SEMICYUC, XXXVI Congreso Nacional de la SEEIUC, 7-10 Jun., 2009.

- Martínez Pozo J., Ruiz Rodríguez J.C., Delgado Téllez de Cepeda A.M., Ribas Ripoll V., Monte Moreno E., Caballero López J., Francisco Salas E., Ruiz Sanmartín A., Bóveda Treviño J.L., "Predicció de mortalitat a la sepsia greu a partir d'un punt de tall a l'escala SOFA", XXX Reunió de la Socitetat Catalana de Medicina Intensiva i Crítica, 19-20 Mar., 2009.

- Ruiz Rodríguez J.C., Caballero López J., Ruiz Sanmartín A., Ribas Ripoll V., Pérez M., Bóveda Treviño J.L., Rello J., "Procalcitonin clearance as a Severe Sepsis and multiorgan dysfunction prognostic biomarker", Med Intensiva. 2012. doi:10.1016/j.medin.2011.11.024.

- Medical Papers (Under Revision):

- Ribas V., Vellido A., Romero E., Ruiz Rodríguez J.C., "Sepsis Mortality Prediction with Quotient Basis Kernels", IEEE Transactions on Biomedical Engineering.

\subsection{Outline for Future Work}

One of the main contributions of this thesis is the provision of evidence for the hypothesis that Generative Models in general and Generative Kernels derived from Algebraic Statistical Models in particular play an important role in the problem of Sepsis prognosis. We have seen that generative models contrast with discriminative models in that the former is a full probabilistic model of all variables, whereas a discriminative model provides a model only for the target variables conditional on the observed variables. Thus a generative model can be used, for example, to simulate values of any variable in the model, whereas a discriminative model allows only sampling of the target variables conditional on the observed quantities. On the other hand, despite the fact that discriminative models do not need to model the distribution of the observed variables, they cannot generally express more complex relationships between the observed and target variables. In this thesis we have only exploited two different approaches stemming from the same framework (i.e., ASM for Graphical Models and ASM for Generative Kernels by means of re-parametrization of a Regular Exponential Family or the derivation of a convex-dual). Beyond the reported research, ASMs for Graphical Models can be used to model other well established Generative Models such as the Restricted Boltzmann Machine, which is the fundamental building block of a Deep Belief Network (DBN). The algebraic properties of the Factor Analysis Model have been studied in [72] and [68] and only recently has it been shown that the RBM for classification is the undirected analogue 
of factor analysis (i.e. they are modelled as 5.2 with weighted links and biased visible and hidden variables) [116]. Moreover, Regular Exponential Families can be generalized by means of Exponential Family Harmoniums [117].

The free energy of an RBM is:

$$
\phi(v, h)=\exp \left(-h^{t} W v+b^{t} v+c^{t} h\right),
$$

where $h$ are the hidden units, $v$ the visible units, $W$ is the transition matrix and $b$ and $c$ correspond to the biases for the visible and hidden layer. The training of a DBN is not obvious and is currently done by means of Contrastive Divergence [117]. It has been shown [116] that by application of the following change of variables,

$$
\gamma_{i}=\exp \left(c_{i}\right) \quad \omega_{i j}=\exp \left(W_{i j}\right) \quad \beta_{j}=\exp \left(b_{j}\right)
$$

the free energy reduces to the following square-free polynomial:

$$
\psi(v, h)=\prod_{i=1}^{k} \gamma_{i}^{h_{i}} \prod_{i=1}^{k} \prod_{j=1}^{n} \omega_{i j}^{h_{i} v_{j}} \prod_{j=1}^{n} \beta_{j}^{v_{j}} .
$$

This re-parametrization means that it is possible to make a robust and efficient implementation of an RBM for building models in general and for Sepsis in particular. Moreover, this also raises the question if this same reparametrization would hold for the multinomial case or more general cases (an outline of a proof for the multinomial case is to model the latter as combination of binomial distributions, expansion to the Gaussian needs to be done by means of the Central Limit Theorem). The work in [118] also shows that all solutions for the RBM (i.e. $W, b$ and $c$ ) lie in an open cone linearity of the tropical morphism. Although the number of valid inference functions for a given RBM is extremely high it is possible to calculate the transitions between the hidden and observed states by means of Tropical Algebra. The emerging field of Tropical Algebra has yielded encouraging results in the study of graphical models in general and Hidden Markov Models in particular [14], since it allows to apply the Viterbi Algorithm to calculate the hidden states of a given/observed sequence. However, it is still necessary to study the generalization capabilities of this approach for the non-binary case. Besides that, it is also necessary to study if it is possible to derive an efficient algorithm to obtain the best inference function from the open cone outlined above (that is, is there a better and alternative algorithm to the currently used Contrastive Divergence?).

Besides these methodological questions, and from a clinical viewpoint, it is necessary to study the generalization capabilities of the indicators presented in this thesis by means of a multi-centric study and set a formal comparison with the most widely used ICU indicators. Also in this regard, we believe that it would be worth applying the methodology proposed in the treatment of Sepsis (like the PROWESS study for Xigris) and also test in a randomized study how the continuation of treatment with statins impacts on the ICU outcome for Sepsis. 


\section{Appendix A}

\section{General Considerations of Topology and Measure Theory}

In this appendix we revise the basic notions of topology $[119,120]$ and measure theory that have been used in this PhD thesis. The principles and notions presented here are used throughout this work and more particularly in the presentation of Gaussian Processes and Discrete Distributions as Regular Exponential Families as well as the derivation of the generative kernels induced by these two families.

Provided that we are working with structured domains that are not necessarily Euclidean it makes sense to take a higher abstraction step and use more general topological spaces. More specifically, we will work with the Radon measure, which is a measure on the $\sigma$-algebra of Borel sets of a Hausdorff topological space that is locally finite and inner regular.

\section{A.1 Topological Spaces}

Definition 51. Topological Space:

Let $X$ be a set and $\mathcal{P}(X)$ the collection of its parts. A topological space $X$ is a collection $\mathcal{F} \subseteq \mathcal{X}$ that contains both $\emptyset$ and $X$ and that is closed under finite intersections and arbitrary unions. The members of $\mathcal{F}$ are called open sets.

Definition 52. Topological Basis:

Given a topological space $X$, a basis for the topology $\mathcal{F}$ is any family of sets $\left\{B_{i}\right\}_{i \in I}$ that generates $\mathcal{F}$ by taking finite intersections and arbitrary unions of its elements.

Definition 53. Continuous Maps:

A map $f$ between two topological spaces $X$ and $Y$ is called continuous if the inverse image of any open set in $Y$ is open in $X$ (i.e. $f^{-1}(V) \in \mathcal{F}_{X} \forall V \in \mathcal{F}$ ).

Definition 54. Compact:

A subset $S$ of $X$ is said to be compact if any open covering of $S$ has a finite sub-covering. 
Remark 6. For any family of open sets $\left\{S_{i}\right\}_{i \in I}$ such that $S \subseteq \bigcup_{i \in I} S_{i}$ there exists a finite subfamily $\left\{S_{i_{1}}, \ldots, S_{i_{n}}\right\}$ such that $S \subseteq S_{i_{1}} \cup \cdots \cup S_{i_{n}}$.

Remark 7. The image of a compact set under a continuous map is a compact set. Continuous maps preserve compactness.

Definition 55. Ordinary Topology in $\mathbb{R}$ :

Let $X=\mathbb{R}$ and define a set $S$ open if any point $x \in S$ belongs to an open interval contained in $S$. Then, a set $C \subseteq X$ is compact iff it is closed and bounded.

Definition 56. Norm:

Let $V$ be a vector space over $\mathbb{C}$ (analogously over $\mathbb{R}$ ). A norm in $V$ is a function $\|\cdot\|: V \rightarrow \mathbb{R}^{+}$that satisfies, for all $\alpha \in \mathbb{C}$ and $u, v \in V$ :

- $\|u\|=0$ iff $u=\mathbf{0}$

- $\|\alpha u\|=|\alpha|\|u\|$

- $\|u+v\| \leq\|u\|+\|u\|$

Definition 57. Ordinary Topology:

Let $V$ be a vector space endowed with a norm. The topology induced by the family of open balls of the form

$$
B_{\epsilon}(u)=\{v \in V:\|v-u\|<\epsilon\}
$$

is called the ordinary topology in $V$.

Definition 58. Banach Space:

If $V$ is complete with respect to its norm (i.e. every Cauchy Sequence has a limit in $V$ ), then $V$ is called a Banach Space.

Definition 59. Inner Product:

Let $V$ be a vector space over $\mathbb{C}$ (analogously in $\mathbb{R}$ ). An inner product in $V$ is a function $\langle\cdot, \cdot\rangle: V \times V \rightarrow \mathbb{C}$ satisfying for all $u, v, w \in V$ and all $\alpha \beta \in \mathbb{C}$ :

- $\langle\alpha u+\beta v, w\rangle=\alpha\langle u, w\rangle+\beta\langle v, w\rangle$

- $\langle u, v\rangle=\overline{\langle v, u\rangle}$

- $\langle v, v\rangle \geq 0$ with equality iff $u=\mathbf{0}$.

Remark 8. Any inner product induces a norm via $\|x\| \equiv\langle x, x\rangle^{\frac{1}{2}}$. Therefore, we can also define a family of open balls $B_{\epsilon}(u)$ and obtain the ordinary topology in $V$.

Definition 60. Metric Space

A metric space is a set $X$ endowed with a metric, i.e., a function $d: X \times X \rightarrow$ $\mathbb{R}^{+}$that satisfies for all $x, y, z \in X$ :

- $d(x, y)=0$ iff $x=y$

- $d(x, y)=d(y, x) \forall x, y \in X$

- $d(x, z) \leq d(x, y)+d(y, z) \forall x, y, z \in X$ 

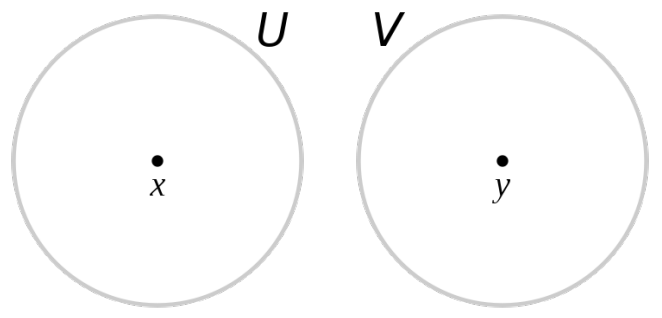

Figure A.1: Two points separated by open sets in a Haussdorff Space

We may also define open balls in a metric space trough:

$$
B_{\epsilon}(x)=\{y \in X: d(x, y)<\epsilon\}
$$

and obtain the ordinary topology as defined above. Defining Cauchy Sequences and completeness with respect to the metric allows characterizing compact sets in $X$ analogously.

Any normed vector space is a metric space, defining $d(x, y) \equiv\|y-x\|$.

Definition 61. Hilbert Space:

A Hilbert space $H$ is a real or complex inner product space that is also a complete metric space with respect to the distance function induced by the inner product.

Definition 62. Haussdorff Space:

A Haussdorff space is a topological space $X$ where any pair of distinct points can be separated by open sets, that is, for any $x, y \in X$ with $x \neq y$ there exist $U, V \in \mathcal{F}$ with $U \cap V=\emptyset$ such that $x \in U$ and $y \in V$.

Haussdorff spaces generalize metric spaces provided that any metric space under the ordinary topology is Hausdorff. An important fact is that, if $X$ is Haussdorff, the any compact subset $C \subseteq X$ is necessarily closed. In particular, any singleton is closed.

\section{A.2 Measures}

Definition 63. Let $X$ be a set:

- A $\sigma$-algebra on $X$ is a collection $\mathcal{M} \subseteq \mathcal{P}(X)$ that contains $\emptyset$ and that is closed under taking complements and countable unions.

- The members of $\mathcal{M}$ are called measurable sets.

- $(X, \mathcal{M})$ is called a measurable space.

If $X$ is endowed with a topology, a natural $\sigma$-algebra is the algebra $\mathcal{B}(X)$ of the borel subsets of $X$, i.e., the algebra generated by the open subsets of $X$. An element of $\mathcal{B}(X)$ is called Borel measurable. 
Definition 64. Positive Measure

A positive measure on a measurable space $(X, \mathcal{M})$ is a map:

$$
\mu: \mathcal{M} \rightarrow[0, \infty]
$$

which is countably additive. A measurable space together with a measure $\mu$ is called a measured space and denoted $(X, \mathcal{M}, \mu)$. A positive measure defined on $\mathcal{B}(X)$ is called Borel measurable.

To define the integral in a measured space $(X, \mathcal{M}, \mu)$, we first consider step functions and then proceed to $\mu$-measurable functions. A step function is a function $\psi: X \rightarrow \mathbb{R}$ that is step with respect to some partition $A_{1}, \ldots, A_{r}$ of some set $A \subseteq X$ of finite measure. The integral of $\psi$ is defined as $\int \psi d \mu=$ $\sum_{r=1}^{r} \mu\left(A_{i}\right) \psi\left(A_{i}\right)$. A function $f: X \rightarrow \mathbb{R}$ is called $\mu$ measurable if it is the point wise limit of a sequence of step functions $\left\{\psi_{n}\right\}_{n \in \mathbb{N}}$ almost everywhere (i.e. any point of $X \backslash Z$ where $Z$ is some set of null measure). In that case, the integral of $f$ is defined as $\int f d \mu=\lim \int \psi_{n} d \mu$. The case $X=\mathbb{R}^{n}$ endowed with a Lebesgue-Borel measure corresponds to the Lebesgue integral.

Definition 65. Radon Measure

Let $X$ be a Haussdorff space. A Radon measure on $X$ is a Borel measure satisfying:

- $\mu(C)<\infty$ for each compact subset $C \subseteq X$,

- $\mu(B)=\sup \{\mu(C): C \subseteq B, C$ compact $\}$ for each $B \in \mathcal{B}(X)$.

We denote the set of all Radon measures on $X$ by $M_{+}(X)$.

Definition 66. Molecular Measures

The support of a Radon measure $\mu$ on $X$ is defined as

$$
\sup (\mu)=\{x \in X: \mu(U)>0 \text { for each neighbourhood of } U \text { of } x\} .
$$

Radon measures with a finite support are called molecular measures. The set of all molecular measures on $X$ is denoted $\mathrm{Mol}_{+}(X)$.

\section{A.3 Entropy and Divergences}

Let $(X, \mathcal{M}, v)$ be a measured space where $X$ is Haussdorff and $v$ is a $\sigma$-finite Radon measure. Let $M_{+}^{h}(X) \subseteq M_{+}^{b}(X)$ denote the set of finite Radon $v$ absolutely continuous measures whose density $f: X \rightarrow \mathbb{R}^{+}$satisfies $\|f \log f\|_{1}<$ $\infty$. Denote by $\frac{d}{d v} M_{+}^{h}(X)$ the set of densities of those measures. The entropy function $h: \frac{d}{d v} M_{+}^{h}(X) \rightarrow \mathbb{R}$ is defined by:

$$
h(f)=-\int_{\mathcal{X}} f \log f d v
$$

where $h(0)=0$ since $\lim _{f \rightarrow 0}-f \log f=\lim _{f \rightarrow 0} \frac{-\log f}{\frac{1}{f}}=0$. 
Remark 9. This definition of entropy generalizes the more traditional notions of discrete and differential entropies. Denote by $M_{+}^{1, h}(X)=M_{+}^{h}(X) \cap M_{+}^{1}(X)$ the set of Radon probability measures with finite entropy. If $X \subseteq \mathbb{R}^{n}, v$ is the Lebesgue-Borel measure, and $P \in M_{+}^{1, h}(X)$ is a probability measure with density $p=\frac{d P}{d v}$, then $h(p)$ reduces to differential entropy:

$$
h(p)=-\int_{\mathcal{X}} p(x) \log p(x) d x .
$$

If instead $X$ is a countable set, $v$ is the counting measure, and $P \in M_{+}^{1, h}(X)$ is a probability measure with probability mass function $x \mapsto p(x)=P(\{x\})$, then $h(p) \equiv H(p)$ is the discrete entropy

$$
H(p)=-\sum_{x \in X} p(x) \log p(x)
$$

Definition 67. Kullback-Leibler Divergence

Let $f$ and $g$ be respectively the densities (with respect to dominating measure $v)$ of measures $\mu_{f}$ and $\mu_{g}$ in $M_{+}^{h}(X)$, such that $\mu_{f}$ is $\mu_{g}$-absolutely continuous (i.e. $\mu_{f}<<\mu_{g}<<v$ ). The Kullback-Leibler divergence (KL) between $f$ and $g$ is defined by:

$$
D(f \| g)=\int_{\mathcal{X}} f \log \frac{f}{g} d v=-h(f)-\int_{\mathcal{X}} f \log g d v .
$$

Remark 10. The Kullback-Leibler Divergence (KL) is not a metric since it is not symmetric and it does not satisfy the triangular inequality.

Remark 11. If $g$ and $f$ are probability densities, the $K L$ divergence can be seen as a dissimilarity measure between the two distributions. The KL divergence satisfies $D(f \| g)=0$ iff $f=g$ almost everywhere.

It is clear that $M_{+}(X)$ and $M_{+}^{b}(X)$ are convex cones, and that $M_{+}^{1}(X)$ is a convex set. By linearity of the integral, so are the respective sets of densities. Therefore, we can talk about "Mixtures of Densities". These may be characterized by the following divergence measure:

Definition 68. Jensen-Shannon Divergence

Let $f_{1}, \ldots, f_{n}$ be densities of measures in $M_{+}^{h}(X)$, and $f=\alpha_{1} f_{1}+\cdots+\alpha_{n} f_{n}$ a mixture defined by coefficients $\alpha_{1}, \ldots, \alpha_{n} \in \mathbb{R}^{+}$. The generalized JensenShannon divergence of $f_{1}, \ldots, f_{n}$ with respect to that mixture is defined by:

$$
J\left(f_{1}, \ldots, f_{n} ; \alpha_{1}, \ldots, \alpha_{n}\right) \equiv h\left(\sum_{i=1}^{n} \alpha_{i} f_{i}\right)-\sum_{i=1}^{n} \alpha_{i} h\left(f_{i}\right),
$$

The restriction of $J$ to probability densities is defined analogously requiring $\sum_{i=1}^{n} \alpha_{i}=1$. The particular case where $n=2$ and $\alpha_{1}=\alpha_{2}=1 / 2$ is simply called Jensen-Shannon divergence between $f$ and $g$ and denoted $J(f \| g)$ :

$$
J(f \| g) \equiv h\left(\frac{f+g}{2}\right)-\frac{h(f)+h(g)}{2} .
$$

The Jensen-Shannon divergence $M_{+}^{1}(X) \times M_{+}^{1}(X) \rightarrow[0, \infty)$ is also defined as a smoothed and centred version of the KL divergence. 
Definition 69. Let $f$ and $g$ be densities of measures in $M_{+}^{1}(X)$ and $p=\frac{f+g}{2}$, then

$$
J(f \| g) \equiv \frac{1}{2} K L(f \| p)+\frac{1}{2} K L(g \| p)
$$

It is well known that $\sqrt{J(f \| g)}$ is a metric. $\sqrt{J(f \| g)}$ is also known to be Hilbertian [121]. A metric $d(x, y)$ is said to be Hilbertian iff $d^{2}(x, y)$ is negative definite [78]. Since $\sqrt{J(f \| g)}$ is a Hilbertian metric, $J(f \| g)$ is n.d. 


\section{Bibliography}

[1] Knaus W.A., Wagner D.P., Draper E.A., Zimmerman J.E., Bergner M., Bastos P.Gl., Sirio C.A., Murphy D.J., Lotring T., Damiano A. The APACHE III prognostic system. Risk prediction of hospital mortality for critically ill hospitalized adults. Chest, 100(6):1619-1936, 1991.

[2] Vincent J.L., Moreno R., Takala J., Willats S., De Mendoça A., Bruining H., Reinhart C.K., Suter P.M., Thijs L.G. The SOFA (sepsis-related organ failure assessment) score to describe organ dysfunction/failure. Crit. Care Med, 22:707-710, 1996.

[3] Martin G.S., Mannino D.M., Eaton S., Moss M. The epidemiology of sepsis in the united states from 1979 through 2000. N Engl J Med., 348:1546$1554,2003$.

[4] Harrison T.R., Kasper D.L., Braunwald E., Fauci A.S., Hauser S.L., Longo D.L., Jameson J.L., Loscalzo J. Harrison's Principles of Internal Medicine 17th Ed. McGraw-Hill Medical Publishing Division, 2008.

[5] Mitchell M., Levy MD. Biomarkers in the Critically Ill Patient, Critical Care Clinics, volume 7(2). W.B. Saunders Company, Elsevier, Philadelphia, 2011.

[6] Sadique Z., Grieve R., Harrison D.A., Cuthbertson B.H., Rowan K.M. Is drotrecogin alfa (activated) for adults with severe sepsis, cost-effective in routine clinical practice? Crit. Care, 15(R228), 2011.

[7] Dellinger R. P., Carlet J. M., Masur H., Gerlach H., Calandra T., Cohen J., Gea-Banacloche J., Keh D., Marshall J. C., Parker M. R., Ramsay G., Zimmerman J. L., Vicent J. L., Levy M. M. Surviving sepsis campaign guidelines for management of severe sepsis and septic shock. Intensive Care Med, 30:536-555, 2004.

[8] Villar J., Cabrera N.E., Casula M., Flores C., Valladares F., Díaz-Flores, Muros M., Slutsky A.S., Kacmarek R.M. Mechanical ventilation modulates TLR4 and IRAK-3 in a non-infectious, ventilator-induced lung injury model. Respir. Res., 11:27, 2010.

[9] Ringwood L., Liwu L. The involvement of the interleukin-1 receptor associated kinases (IRAKs) in cellular signaling networks controlling inflammation. Cytokine, 42:1-7, 2008. 
[10] Herrera M.T., Toledo C., Valladares F., Muros M., Díaz-Flores L., Flores C., Villar J. Positive end-expiratory pressure modulates local and systemic inflammatory responses in a sepsis-induced lung injury model. Intensive Care Med, 29:1345-1353, 2003.

[11] Cohen J. The immunopathogenesis of sepsis. Nature, 420:885-891, 2002.

[12] Williams D.L., Ha T., Li C., Kalbfleisch J.H., Schweitzer J., Vogt W., Browder W. Modulation of tissue toll-like receptor 2 and 4 during the early phases of polymicrobial sepsis correlates with mortality. Crit Care Med, 31:1808-1818, 2003.

[13] Lukaszewski R.A., Yates A.M., Jackson M.C., Swingler K., Scherer J.M., Simpson A.J., Sadler P., McQuillan P., Titball R.W., Brooks T.J.G., Pearce M.J. Mechanical ventilation modulates TLR4 and IRAK-3 in a non-infectious, ventilator-induced lung injury model. Respir. Res., 11:27, 2010 .

[14] Pachter L., Sturmfels B. Algebraic Statistics for Computational Biology. Cambridge University Press, 2005.

[15] Majno G. The ancient riddle of $\sigma \tilde{\eta} \psi \iota \varsigma$ (sepsis). J Infec Dis., 163(5):937945,1991

[16] Frazer R. (ed), Sir James George Fraser. The Golden Bough: A Study in Magic and Religion (Oxford World's Classics). Oxford Paperbacks, Reissue Edition 2009.

[17] Littré É. Oeuvres complètes d'Hippocrate Tome 10. Adamant Media Corporation, 2001.

[18] Lucius Mestrius Plutarchus. Plutarch's Moralia. The Online Library of Liberty, http://oll.libertyfund.org/, 1878.

[19] Renehan R. A rare surgical procedure in plutarch. The Classical Quaterly, New Series, 50(1):223-229, 2000.

[20] http://www.sepsis-gesellschaft.de.

[21] American College of Chest Physicians/Society of Critical Care Medicine Consensus Conference. Definitions for sepsis and organ failure and guidelines for the use of innovative therapies in sepsis. Crit Care Med., 20:864874,1992 .

[22] Levy M.M., Fink M.P., Marshall J.C., Edward Angus A., Cook D., Cohen J., Opal S.M., Vincent J.L., Ramsay G. for the International Sepsis Definitions Conference (2003). 2001 SCCM/ESICM/ACCP/ATS/SIS International sepsis definitions conference. Int. Care Med., 29:530-538, 2003.

[23] Levy M.M., Macias W.L., Vincent J.L., Russell J.A., Silva E., Trzaskoma B., Williams D. Early changes in organ function predict eventual survival in severe sepsis. Crit. Care Med, 31:243-249, 2005. 
[24] Kajdacsy-Balla A.C., Moreira Andrade F., Moreno R., Artigas A., Cantraine F., Vincent J.L. Use of the sequential organ failure assessment score as a severity score. Intensive Care Med, 33(10):2194-2201, 2005.

[25] Knaus W. A., Draper E. A., Wagner D. P., Zimmerman J. E. APACHE II: A severity of disease classification system. Crit. Care Med., 13:818-829, 1985.

[26] Le Gall J.R., Neuman F.H., Bleriot J.P., Fulgencio J.P., Garrigues B., Gouzes C., Lepage E., Moine P., Villers D. Mortality prediction using SAPS II: an update for French intensive care units. Crit. Care, 9(6):R645R652, 2005.

[27] Astiz M., Tilly E., Rackow E.D., Weil M.H. Peripheral vascular tone in sepsis. Chest, 99:1072-1075, 1991.

[28] Toweill D., Sonnenthal K., Kimberly B., Lai S., Goldstein B. Linear and nonlinear analysis of hemodynamic signals during sepsis and septic shock. Crit. Care Med., 28(6):2051-2057, 2000.

[29] Goldman D., Bateman R.M., Ellis C.G. An experiment-based model of oxygen transport in capillary networks under normal and septic conditions. In EMBS/BMES Conference, 2002. Proceedings of the Second Joint, volume 2, pages 1517-1518, 2002.

[30] Ross J.J., Mason D.G., Paterson I.G., Linkens D.A., Edwards N.D. Development of a knowledge-based simulator for haemodynamic support of septic shock. In Simulation in Medicine (Ref. No. 1998/256), IEEE Colloquium on, pages 3/1-3/4, 1998.

[31] Denai M., Mahfouf M., Ross J. A fuzzy decision support system for therapy administration in cardiovascular intensive care patients. In FUZZIEEE 2007. IEEE International, pages 1-6, 2007.

[32] Ce Xu, Zhiguo Ye, Qin Gao, Qixian Shan, Qiang Xia, Borreau J.P. The relationship of ventricular dynamics and mitochondrial nitric oxide synthase activity in septic shock models. In IEEE-EMBS 2005. 27th Annual International Conference of the, pages 2280-2282, 2005.

[33] Gonzalez C.A., Villanueva C., Othman S., Sacristan E. Therapy guided by gastric impedance spectroscopy in a septic shock model in pigs. In IEMBS '04. 26th Annual International Conference of the IEEE, volume 3, pages 2307-2310, 2004.

[34] Gonzalez C.A., Villanueva C., Othman S., Sacristan E. Classification of impedance spectra for monitoring ischemic injury in the gastric mucosa in a septic shock model in pigs. In IEMBS '03. 25th Annual International Conference of the IEEE, volume 3, pages 2269-2272, 2003.

[35] Stacey M., McGregor C., Tracy M. An architecture for multi-dimensional temporal abstraction and its application to support neonatal intensive care. In EMBS 2007. 29th Annual International Conference of the IEEE, pages $3752-3756,2002$. 
[36] Paetz J. Intersection based generalization rules for the analysis of symbolic septic shock patient data. In ICDM 2002. Proceedings. 2002 IEEE International Conference on, pages 673-676, 2002.

[37] Paetz H. Metric rule generation with septic shock patient data. In ICDM 2001, Proceedings IEEE International Conference on, pages 637$638,2001$.

[38] Schuh Ch. J. Sepsis and septic shock analysis using neural networks. In NAFIPS '0\%. Annual Meeting of the, pages 650-654, 2007.

[39] Duhamel A., Beuscart R., Demongeot J., Mouton Y. SES (septicemia expert system): knowledge validation from data analysis. In Engineering in Medicine and Biology Society, 1988. Proceedings of the Annual International Conference of the IEEE, volume 3, pages 1400-1401, 1988.

[40] Beuscart R., Duhamel A., Moussu L., Quenton S. Using clinical datafiles to improve expert systems efficiency. In Engineering in Medicine and Biology Society, 1989. Images of the Twenty-First Century., Proceedings of the Annual International Conference of the IEEE Engineering in, 1989.

[41] Kim J., Blum J., Scott C. Temporal features and kernel methods for predicting sepsis in postoperative patients. http://www.eecs.umich.edu/ cscott/pubs/sepsisTR.pdf, 2010.

[42] Shu-Li Wang, Fan Wu, Bo-Hang Wang. Prediction of severe sepsis using svm model. Advances in Experimental Medicine and Biology Series, 680(1):75-81, 2010.

[43] Rangel-Frausto M.S, Pittet D., Costigan M., Hwang T., Davis C., Wenzel R.P. The natural history of the sistemic inflammatory response syndrome (SIRS). a prospective study. JAMA, 273:117-123, 1995.

[44] Pittet D., Rangel-Frausto S., Li N., Tarara D., Costigan M., Remple L., Jebson P., Wenzel R.P. Systemic inflammatory response syndrome, sepsis, severe sepsis and septic shock: incidence, morbidities and outcome in surgical ICU patients. Intensive Care Med, 21:302-309, 1995.

[45] Sankoff J.D., Goyal M., Gaieski D.F., Dietch K., Davis C.B., Sabel A.L., Haukoos J.S. Validation of the mortality in emergency department sepsis (MEDS) score in patients with the systemic inflammatory response syndrome (SIRS). Crit Care Med, 36(2):1-6, 2008.

[46] Knaus W.A, Draper E.A, Wagner D.P, Zimmerman J.E. Prognosis in acute organ-system failure. Ann Surg, 202:685-693, 1985.

[47] Le Gall J.R., Klar J., Lemeshow S. How to assess organ dysfunction in the intensive care unit? The logistic organ dysfunction (LOD) system. Sepsis, 1:45-47, 1997.

[48] Moreno R.P, Metnitz B., Adler L., Hoechtl A., Baure P., Metnitz P.G.H. Sepsis mortality prediction based on predisposition, infection and response. Intensive Care Med, 34:496-504, 2008. 
[49] Rubulotta F., Marshall J.C, Ramsay G., Nelson D., Levy M., Williams M. Predisposition, insult/infection, response and organ dysfunction: a new model for staging severe sepsis. Crit Care Med, 37:1329-1335, 2009.

[50] Brause R., Hamker F., Paetz J., Jain L.C. (ed). Septic Shock Diagnosis by Neural Networks and Rule Based Systems", Computational Intelligence Techniques in Medical Diagnosis and Prognosis. Springer Verlag, 2001.

[51] Brause R., Hanisch E., Paetz J., Arlt B. Neuronal networks for sepsis prognosis - the medan project. Journal für Anästhesie und Intensivbehandlung, 11(1):40-43, 2004.

[52] Tang C.H.H., Middleton P.M., Savkin A.V., Chan G.S.H., Bishop S., Lovell N.H. Non-invasive classification of severe sepsis and systemic inflammatory response syndrome using a nonlinear support vector machine: a preliminary study. Physiol. Meas., 31:775-793, 2010.

[53] Brause R.W. About adaptive state knowledge extraction for septic shock mortality prediction. In (ICTAI 2002). Proceedings. 14th IEEE International Conference on, volume ., pages 3-8, 2002.

[54] Giuliano K.K. Physiological monitoring for critically ill patients: testing a predictive model for the early detection of sepsis. Am. J. Crit. Care, 16(2):122-130, 2007.

[55] Moorman J., Randall L., Douglas E., Griffin M.P. Heart rate characteristics monitoring for neonatal sepsis. Biomedical Engineering, IEEE Transactions on, 53(1):126-132, 2006.

[56] Ely E.W., Laterre P.F., Angus D.C., Helterbrand J.D., Levy H., Dhainaut J.F., Vincent J.L., Macias W.L., Bernard G.R.,. Drotrecogin alfa (activated) administration across clinically important subgroups of patients with severe sepsis. Crit. Care, 31(1):12-19, 2003.

[57] G. Pistone, E. Riccomagno, and H.P. Wynn. Algebraic Statistics: Computational Commutative Algebra in Statistics, volume 89 of Monographs on Statistics and Applied Probability. Chapman and Hall, CRC Press, Boca Raton, 2001.

[58] Drton M., Sullivant S. Algebraic statistical models. Statist. Sinica., 17:1273-1297, 2007.

[59] Marinari M.G., Möller H.M., Mora T. Gröbner bases of ideals definedby functionals with an application to ideals of projective points. Appl. Algebra Engrg. Comm. Comput, 4:105-145, 1993.

[60] http://apcocoa.org.

[61] CoCoATeam. CoCoA: a system for doing Computations in Commutative Algebra. Available at http://cocoa.dima.unige.it.

[62] J. Abbott, A. Bigatti, M. Kreuzer, and L. Robbiano. Computing ideals of points. JSYMC, 30(4):341-356, 2000. 
[63] Giglio B., Riccomagno E., Wynn H. Gröbner basis strategies in regression. Journal of Applied Statistics, 27(7):923-938, 2000.

[64] Bartle G. The Elements of Integration and Lebesgue Measure. Wiley Interscience, Canada, 1995.

[65] Rudin W. Real and Complex Analysis. McGraw-Hill, 1987.

[66] Brown L.D. Fundamentals of Statistical Exponential Families with Applications in Statistical Decision Theory. Institute of Mathematical Statistics Lecture Notes - Monograph Series - Vol 9, Hayward, California, 1986.

[67] McCullagh P. What is a statistical model? The Annals of Statistics, 30(5):1225-1310, 2002.

[68] Drton M., Sturmfels B., Sullivant S. Lectures on Algebraic Statistics. Birkhäuser, Basel, Boston, Berlin, 2009.

[69] Lauritzen S. Graphical Models. Oxford University Press, 1996.

[70] Kindermann R., Snell J.L. Markov Random Fields and Their Applications. American Mathematical Society, 1980.

[71] Bishop C.M. Pattern Recognition and Machine Learning. Springer, Cambridge, U.K., 2006.

[72] Drton M., Sturmfels B., Sullivant S. Algebraic factor analysis: tetrads, pentads and beyond. Probab. Theory Relat. Fields, 138:463-493, 2007.

[73] Shölkopf B., Smola A.J. Learning with Kernels. The MIT Press, 2002.

[74] Shoenberg I.J. Metric spaces and positive definite functions. Transactions of the American Mathematical Society, 44(3), 1938.

[75] Hein M., Bousquet O. Hilbertian metrics and positive definite kernels on probability measures. Max Planck Institute for Biological Cybernetics Technical Report, 126, 2004.

[76] Agarwal A., Daumé III H. Generative kernels for exponential families. In International Conference on Artificial Intelligence and Statistics (AISTATS), 2011.

[77] Berg C. and Christensen J.P.R and Ressel P. Harmonic analysis on semigroups. Springer-Verlag, New-York, 1984.

[78] Schoenberg I.J. Metric spaces and positive definite functions. In Transactions of the American Mathematical Society, volume 44, pages 522-536, 1938.

[79] Shawe-Taylor J., Cristianini N. Kernel Methods for Pattern Analysis. Cambridge University Press, Cambridge, U.K., 2004.

[80] Kullback S., Leibler R.A. On information and sufficiency. Annals of Mathematical Statistics, 22:49-86, 1951. 
[81] Friedman J., Hastie T., Tibshirani R. The Elements of Statistical Learning. Springer-Verlag, 2008.

[82] Breiman L. and Friedman J. and Stone C.J. Classification and Regression Trees. Chapman and Hall/CRC, 1984.

[83] Shawe-Taylor J. and Cristianini N. Kernel Methods for Pattern Analysis. Cambridge University Press, 2006.

[84] http://www.tristanfletcher.co.uk/SVM\%20Explained.pdf.

[85] Tipping M. Sparse bayesian learning and the relevance vector machine. Journal of Machine Learning Research, 1:211-244, 2001.

[86] http://www.tristanfletcher.co.uk/RVM\%20Explained.pdf.

[87] I. T Jolliffe. Principal Component Analysis. Springer, 2002.

[88] Lee D.D., Seung S.H. Learning the parts of objects by non-negative matrix factorization. Nature, 6755(401):788-791, 1999.

[89] Rubin D.B., Thayer D.T. EM Algorithms for ML factor analysis. Psychometrika, 47:69-76, 1982.

[90] Almog Y., Novack V., Eisinger M., Porath A., Novack L., Gilutz H. The effect of statin therapy on infection-related mortality in patients with atherosclerotic diseases. Crit. Care Med, 35:372-378, 2007.

[91] Chopra V., Flanders S.A. Does statin use improve pneumonia outcomes? Chest, 136:1381-1388, 2009.

[92] Liappis A.P., Kan V.L., Rochester C.G., Simon G.L. The effect of statins on mortality in patients with bacteriemia. Clinical Infectious Diseases, 33:1352-1357, 2001.

[93] Gao F., Linhartova L., Johnston M., Thickett D.R. Statins and sepsis. Br J Anaesth, 100:288-298, 2008.

[94] Thomsen R.W., Hundborg H.H., Johnsen S.P.J., Pedersen L., Sorensen H.T., Schonheyder H.C., Lervang H.H. Statin use and mortality within 180 days after bacteremia: A population-based cohort study. Crit Care Med, 34:1080-1086, 2006.

[95] Hackam D.G., Mamdani M., Li P., Redelmeier D.A. Statins and sepsis in patients with cardiovascular disease: a population-based cohort analysis. Lancet, 367:413-418, 2006.

[96] Almog Y. Statins, inflammation, and sepsis. Chest, 124:740-743, 2003.

[97] Gupta R., Plantinga L.C., Fink N.E., Melamed M.L., Coresh J., Fox C.S., Levin N.W., Powe N.R. Statin use and hospitalization for sepsis in patients with chronic kidney disease. JAMA, 297:1455-1464, 2007.

[98] Tleyjeh I.M., Kashour T., Hakim F.A., Zimmerman V.A., Erwin P.J., Sutton A.J., Ibrahim T. Statins for the prevention and treatment of infections. a systematic review and meta-analysis. Arch Intern Med, 169:1658-1667, 2009. 
[99] Christensen S., Thomsen R.W., Johansen M.B., Pedersen L., Jensen R., Larsen K.M., Larsson A., Tonnesen E., Sorensen H.T. Preadmission statin use and one-year mortality among patients in intensive care. a cohort study. Crit Care, 14:R29, 2010.

[100] Schmidt H., Hennen R., Keller A., Russ M., Muller-Werdan U., Werdan K., Buerke M. Association of statin therapy and increased survival in patients with multiple organ dysfunction syndrome. Intensive Care Med, $32: 1248-1251,2006$.

[101] Thomsen R.W., Riis A., Kornum J.B., Christensen S., Johnsen S.P., Sorensen H.T. Preadmission use of statins and outcomes after hospitalization with pneumonia: population-based cohort study of 29,900 patients. Arch Intern Med, 168:2081-2087, 2008.

[102] Majumdar S.R., McAlister F.A., Eurich D.T., Padwal R.S., Marrie T.J. Statins and outcomes in patients admitted to hospital with community acquired pneumonia: population based prospective cohort study. $B M J$, 333(7576):999-1001, 2006.

[103] Kapoor A.S., Kanji H., Buckingham J., Devereaux P.J., McAlister F.A. Strength of evidence for perioperative use of statins to reduce cardiovascular risk: systematic review of controlled studies. BMJ, 333:1149-1156, 2006.

[104] Bellazzi, R., Zupan, B. Predictive data mining in clinical medicine: Current issues and guidelines. International Journal of Medical Informatics, $77: 81-97,2008$.

[105] Hammersley J.M and P. Clifford. Markov Fields on Finite Graphs and Lattices. http://www.statslab.cam.ac.uk/ grg/books/hammfest/hammcliff.pdf, 1971.

[106] Golub, G. H., Reinsch, C. Singular value decomposition and least squares solutions. Numer Math, 14(5):403-420, 1970.

[107] Lisboa P.J.G., Vellido A., Martín J.D. Computational intelligence in biomedicine: Some contributions. In In Procs. of the $18^{\text {th }}$ European Symposium on Artificial Neural Networks (ESANN), volume ., pages 429-438, 2010.

[108] Paliwal, M., Kumar. U.A. Neural networks and statistical techniques: A review of applications. Expert Systems with Applications, 36(1):2-17, 2009.

[109] Kurt, I., Ture, M., Kurum, A.T. Comparing performances of logistic regression, classification and regression tree, and neural networks for predicting coronary artery disease. Expert Systems with Applications, 34(1):366$374,2008$.

[110] Johnson R.A., Wichern D.W. Applied Multivariate Statistical Analysis (6th Edition). Prentice Hall, 2007. 
[111] Lisboa P.J.G., Vellido A., Tagliaferri R., Napolitano F., Ceccarelli M. Data mining in cancer research. IEEE Computational Intelligence Magazine, 5(1):14-18, 2010.

[112] Wong D.T., Crofts S.L., Gomez M., McGuire G.P., Byrick R.J. Evaluation of predictive ability of APACHE II system and hospital outcome in Canadian intensive care unit patients. Crit Care Med., 23(7):1177-1183, 1995.

[113] Wong D.T., Crofts S.L., Gomez M., McGuire G.P., Byrick R.J. Predicting hospital mortality using apache ii scores in neurocritically ill patients: a prospective study. J. Neurol., 256:1427-1433, 2009.

[114] van der Maaten L. Learning discriminative fisher kernels. In Proc. ICML2011, pages 217-224, 2011.

[115] Massey F.J. The Kolmogorov-Smirnov test for goodness of fit. Journal of the American Statistical Association, 253(46):68-78, 1951.

[116] Cueto M. A., Morton J., Sturmfels B. Geometry of the restricted boltzmann machine. Contemporary Mathematics, 506:135-153, 2010.

[117] Welling M., Rosen-Zvi M., Hinton G.E. Exponential family harmoniums with an application to information retrieval. In Advances in Neural Information Processing Systems 17 [Neural Information Processing Systems, NIPS 2004, December 13-18, 2004, Vancouver, British Columbia, Canada], 2004.

[118] Sturmfels B. Speyer D. Tropical mathematics. Mathem. Magazine, 82:163$173,2009$.

[119] W. Massey. A basic course in Algebraic Topology. Springer-Velag, 1999.

[120] Eidelman Y. and Milman V. and Tsolomitis A. Functional Analysis: an introduction. American Mathematical Society, Rhode Island, 2004.

[121] Fuglede B., Topsoe, F. Jensen-Shannon divergence and Hilbert space embedding. In Information Theory, 2004. ISIT 2004. Proceedings. International Symposium on, page 31, june-2 july 2004. 